\title{
POISSON KERNEL CHARACTERIZATION OF REIFENBERG FLAT CHORD ARC DOMAINS
}

\author{
By CARlos E. KENIG $^{1}$ AND TATIANA TORO ${ }^{2}$
}

\begin{abstract}
In this paper we prove the conjecture stated by the authors in Free boundary regularity for harmonic measures and Poisson kernels (Ann. of Math. 150 (1999) 369-454) concerning the free boundary regularity problem for the Poisson kernel below the continuous threshold. We show that if $\Omega$ is a Reifenberg flat chord arc domain, and the logarithm of the Poisson kernel has vanishing mean oscillation then the unit normal vector to the boundary also has vanishing mean oscillation.
\end{abstract}

○ 2003 Éditions scientifiques et médicales Elsevier SAS

RÉSUMÉ. - Dans cet article, on démontre la conjecture proposée par les auteurs dans Free boundary regularity for harmonic measures and Poisson kernels (Ann. of Math. 150 (1999) 369-454) concernant la régularité de la frontière libre pour le noyau de Poisson au-dessous du seuil de continuité. On prouve que si $\Omega$ est un domaine corde-arc Reifenberg plat tel que le logarithme du noyau de Poisson appartienne à VMO, alors le vecteur unitaire normal à la frontière appartient aussi à VMO.

○ 2003 Éditions scientifiques et médicales Elsevier SAS

\section{Introduction}

The main goal of this paper is to present a general blow up argument (see Section 4) which combines geometric and analytic information about the free boundary regularity problem for the Poisson kernel. This technique allows us to provide a complete characterization of Reifenberg flat chord arc domains via potential theory. In particular we prove the conjecture stated in [18], and show that the "weak" regularity of the Poisson kernel of a domain fully determines the geometry of its boundary. Namely we show that if $\Omega$ is a $\delta$-Reifenberg flat chord arc domain for $\delta>0$ small enough, and the logarithm of its Poisson kernel has vanishing mean oscillation then the unit normal vector to the boundary also has vanishing mean oscillation. In our context the mean oscillation of the logarithm of the Poisson kernel, or of the unit normal vector replace stronger notions of regularity. As in Alt and Caffarelli's work (see [1]) we show that at "flat points" of the boundary, the oscillation of the Poisson kernel controls the geometry of the boundary. The difference between our work and the work in [1] is that we measure the oscillation in an integral sense (BMO estimates) while they do so in a pointwise sense (Hölder estimates).

We now introduce formally the definitions needed to state our main results. We indicate how the main theorem follows from the other results, and sketch briefly the contents of each section of the paper. We always assume that $n \geqslant 2$.

\footnotetext{
${ }^{1}$ The author was partially supported by the NSF.

${ }^{2}$ The author was partially funded by the NSF and the Alfred P. Sloan Foundation. 
DEFINITION 1.1. - Let $\Sigma \subset \mathbb{R}^{n+1}$ be a locally compact set, and let $\delta>0$. We say that $\Sigma$ is $\delta$-Reifenberg flat if for each compact set $K \subset \mathbb{R}^{n+1}$, there exists $R_{K}>0$ such that for every $Q \in K \cap \Sigma$ and every $r \in\left(0, R_{K}\right]$ there exists an $n$-dimensional plane $L(Q, r)$ containing $Q$ such that

$$
\frac{1}{r} D[\Sigma \cap B(Q, r), L(Q, r) \cap B(Q, r)] \leqslant \delta .
$$

Here $B(Q, r)$ denotes the $(n+1)$-dimensional ball of radius $r$ and center $Q$, and $D$ denotes the Hausdorff distance.

Recall that for $A, B \subset \mathbb{R}^{n+1}$,

$$
D[A, B]=\sup \{d(a, B): a \in A\}+\sup \{d(b, A): b \in B\} .
$$

Note that the previous definition is only significant for $\delta>0$ small. This notion was initially introduced by Reifenberg who proved the following remarkable theorem.

THEOREM [21,23]. - There exists $\delta>0$ depending only on $n$ so that if $\Sigma$ is $\delta$-Reifenberg flat then locally $\Sigma$ is a topological disc.

We denote by

$$
\theta(Q, r)=\inf _{L}\left\{\frac{1}{r} D[\Sigma \cap B(Q, r), L \cap B(Q, r)]\right\},
$$

where the infimum is taken over all $n$-planes containing $Q$.

DEFINITION 1.2. - Let $\Sigma \subset \mathbb{R}^{n+1}$, we say that $\Sigma$ is Reifenberg flat with vanishing constant if it is $\delta$-Reifenberg flat for some $\delta>0$ and for each compact set $K \subset \mathbb{R}^{n+1}$

$$
\lim _{r \rightarrow 0} \sup _{Q \in \Sigma \cap K} \theta(Q, r)=0
$$

DEFINITION 1.3. - A measure $\mu$ in $\mathbb{R}^{n+1}$ is said to be Ahlfors regular if there exists $C>1$ such that for $Q \in \operatorname{spt} \mu$ and $r>0$

$$
C^{-1} r^{n} \leqslant \mu(B(Q, r)) \leqslant C r^{n} .
$$

DEFINITION 1.4. - Let $\Omega \subset \mathbb{R}^{n+1}$ be a set of locally finite perimeter (see [7]), $\partial \Omega$ is said to be Ahlfors regular if the surface measure to the boundary, i.e., the restriction of the $n$-dimensional Hausdorff measure to $\partial \Omega, \sigma=\mathcal{H}^{n}\llcorner\partial \Omega$, is Ahlfors regular.

DEFINITION 1.5. - Let $\Omega \subset \mathbb{R}^{n+1}$. We say that $\Omega$ has the separation property if for each compact set $K \subset \mathbb{R}^{n+1}$ there exists $R>0$ such that for $Q \in \partial \Omega \cap K$ and $r \in(0, R]$ there exists an $n$-dimensional plane $\mathcal{L}(Q, r)$ containing $Q$ and a choice of unit normal vector to $\mathcal{L}(Q, r)$, $\overrightarrow{n_{Q, r}}$ satisfying

$$
\mathcal{T}^{+}(Q, r)=\left\{X=(x, t)=x+t \overrightarrow{n_{Q, r}} \in B(Q, r): x \in \mathcal{L}(Q, r), t>\frac{1}{4} r\right\} \subset \Omega
$$

and

$$
\mathcal{T}^{-}(Q, r)=\left\{X=(x, t)=x+t \overrightarrow{n_{Q, r}} \in B(Q, r): x \in \mathcal{L}(Q, r), t<-\frac{1}{4} r\right\} \subset \Omega^{c}
$$


Moreover if $\Omega$ is an unbounded domain we also require that $\mathbb{R}^{n+1} \backslash \partial \Omega$ divide $\mathbb{R}^{n+1}$ into two distinct connected components $\Omega$ and int $\Omega^{c} \neq \emptyset$.

The notation $(x, t)=x+t \overrightarrow{n_{Q, r}}$ is used to denote a point in $\mathbb{R}^{n+1}$. The first component, $x$, of the pair belongs to an $n$-dimensional affine space whose unit normal vector is $\overrightarrow{n_{Q, r}}$. The second component $t$ belongs to $\mathbb{R}$. From the context it will always be clear what affine hyperplane $x$ belongs to, and what the orientation of the unit normal vector is.

DEFINITION 1.6. - Let $\delta \in\left(0, \delta_{n}\right)$, where $\delta_{n}$ is chosen appropriately (see note below) and let $\Omega \subset \mathbb{R}^{n+1}$. We say that $\Omega$ is a $\delta$-Reifenberg flat domain or a Reifenberg flat domain if $\Omega$ has the separation property and $\partial \Omega$ is $\delta$-Reifenberg flat. Moreover if $\Omega$ is an unbounded domain we also require that

$$
\sup _{r>0} \sup _{Q \in \partial \Omega} \theta(Q, r)<\delta_{n} .
$$

When we consider $\delta$-Reifenberg flat domains in $\mathbb{R}^{n+1}$ we assume that $\delta_{n}>0$ is small enough, in order to ensure that we are working on NTA domains (see definition in Appendix A, see also [14] and [19, Theorem 3.1]).

DEFINITION 1.7. - A set $\Omega \subset \mathbb{R}^{n+1}$ is said to be a Reifenberg flat domain with vanishing constant if $\Omega$ is a Reifenberg flat domain, and for every compact set $K \subset \mathbb{R}^{n+1}$

$$
\lim _{r \rightarrow 0} \sup _{Q \in \partial \Omega \cap K} \theta(Q, r)=0 .
$$

DEFINITION 1.8. - A set of locally finite perimeter $\Omega \subset \mathbb{R}^{n+1}$ (see [7]) is said to be a chord arc domain, if $\Omega$ is an NTA domain whose boundary is Ahlfors regular.

DEFINITION 1.9. - Let $\delta \in\left(0, \delta_{n}\right)$. A set of locally finite perimeter $\Omega \subset \mathbb{R}^{n+1}$ is said to be a $\delta$-Reifenberg flat chord arc domain, if $\Omega$ is a $\delta$-Reifenberg flat domain whose boundary is Ahlfors regular.

Remarks. - (1) Since $\Omega$ is a $\delta$-Reifenberg flat domain with $\delta>0$ small enough, then for each compact set $K \subset \mathbb{R}^{n+1}$ so that $\partial \Omega \cap K \neq \emptyset$ there exists $R_{K}>0$ so that for every $Q \in \partial \Omega \cap K$ and every $r \in\left(0, R_{K}\right)$ there exists an $n$-plane $L(Q, 2 r)$ containing $Q$ and such that

$$
\begin{aligned}
& \frac{1}{2 r} D[\partial \Omega \cap B(Q, 2 r) ; L(Q, 2 r) \cap B(Q, 2 r)] \leqslant 2 \delta, \\
& \{X=(x, t)=x+t \vec{n}(Q, 2 r): x \in L(Q, 2 r), t>4 \delta r\} \cap B(Q, 2 r) \subset \Omega,
\end{aligned}
$$

and

$$
\{X=(x, t)=x+t \vec{n}(Q, 2 r): x \in L(Q, 2 r), t<-4 \delta r\} \cap B(Q, 2 r) \subset \Omega^{c} .
$$

Here $\vec{n}(Q, 2 r)$ denotes the appropriate unit normal vector to $L(Q, 2 r)$, where we choose $L(Q, 2 r)$ to be the "best" possible approximating $n$-plane to $\partial \Omega$ at $Q$ and at radius $2 r$. (See Remark 1.1 in [18].)

(2) By Remark 4.2 in [18] we have that if $\Omega$ is a set of locally finite perimeter which is a Reifenberg flat domain then the topological boundary of $\Omega$ and its measure theoretic boundary agree.

DEFINITION 1.10. - Let $\delta \in\left(0, \delta_{n}\right)$. A set of locally finite perimeter $\Omega$ (see [7]) is said to be a $\delta$-chord arc domain or a chord arc domain with small constant if $\Omega$ is a $\delta$-Reifenberg flat domain, 
$\partial \Omega$ is Ahlfors regular and for each compact set $K \subset \mathbb{R}^{n+1}$ there exists $R>0$ so that

$$
\sup _{Q \in \partial \Omega \cap K}\|\vec{n}\|_{*}(Q, R)<\delta .
$$

Here $\vec{n}$ denotes the unit normal vector to the boundary,

$$
\|\vec{n}\|_{*}(Q, R)=\sup _{0<s<R}\left(\oint_{B(Q, s)}\left|\vec{n}-\overrightarrow{n_{Q, s}}\right|^{2} d \sigma\right)^{\frac{1}{2}}
$$

and $\overrightarrow{n_{Q, s}}=\oint_{B(Q, s)} \vec{n} d \sigma$

We only use the notation $\delta$-Reifenberg flat domain, $\delta$-Reifenberg flat chord arc domain or $\delta$-chord arc domain when we want to emphasize the dependence on $\delta$, otherwise we simply refer to them as Reifenberg flat domain, Reifenberg flat chord arc domain or chord arc domain with small constant. Note that a chord arc domain with small constant is a Reifenberg flat chord arc domain.

DEFINITION 1.11. - A set of locally finite perimeter is said to be a chord arc domain with vanishing constant if it is a chord arc domain with small constant and for each compact set $K \subset \mathbb{R}^{n+1}$

$$
\lim _{r \rightarrow 0} \sup _{Q \in \partial \Omega \cap K}\|\vec{n}\|_{*}(Q, r)=0 .
$$

We now present the definition of bounded (resp. vanishing) mean oscillation functions on the boundary of a chord arc domain $\Omega$; i.e., $\mathrm{BMO}(\partial \Omega)$ (resp. $\operatorname{VMO}(\partial \Omega)$ ).

DEFINITION 1.12. - Let $\Omega \subset \mathbb{R}^{n+1}$ be a chord arc domain. Let $f \in L_{\text {loc }}^{2}(d \sigma)$, we say that $f \in \operatorname{BMO}(\partial \Omega)$ if

$$
\|f\|_{*}=\sup _{r>0} \sup _{Q \in \partial \Omega}\left(f_{B(Q, r)}\left|f-f_{Q, r}\right|^{2} d \sigma\right)^{\frac{1}{2}}<\infty .
$$

Here $f_{Q, r}=f_{B(Q, r)} f d \sigma$, and $\sigma=\mathcal{H}^{n}\llcorner\partial \Omega$.

DEFINITION 1.13. - Let $\Omega \subset \mathbb{R}^{n+1}$ be a chord arc domain. We denote by $\operatorname{VMO}(\partial \Omega)$ the closure in $\operatorname{BMO}(\partial \Omega)$ of the set of uniformly continuous bounded functions defined on $\partial \Omega$.

The reader will remark that Definition 1.13 is slightly different than the one used in [18] (see Definition 1.8 in [18]). These 2 definitions coincide in the case when $\Omega$ is bounded. In the case when $\Omega$ is unbounded, Definition 1.13 above provides good control on the behavior of $f$ in large balls (see discussion below). This is not the case for the definition used in [18].

Let $\Omega$ be a Reifenberg flat chord arc domain (either bounded or unbounded), and let $X \in \Omega$; then the harmonic measure with pole at $X, \omega^{X}$ and $\sigma=\mathcal{H}^{n}\llcorner\partial \Omega$ are mutually absolutely continuous (see [4] and [25]). The Radon-Nikodym theorem ensures that the corresponding Poisson kernel

$$
k_{X}(Q)=\frac{d \omega^{X}}{d \sigma}(Q)=\frac{\partial G(X,-)}{\partial n}(Q) \in L_{\mathrm{loc}}^{1}(d \sigma) .
$$

Here $G(X,-)$ denotes the Green's function of $\Omega$ with pole at $X$ and $\frac{\partial}{\partial n}=\nabla \cdot \vec{n}$ denotes the normal derivative at the boundary. We prove that if $\Omega$ is a Reifenberg flat chord arc domain, and $\log k_{X} \in \operatorname{VMO}(d \sigma)$ then $\Omega$ is a Reifenberg flat domain with vanishing constant.

$4^{\text {e }}$ SÉRIE - TOME $36-2003-\mathrm{N}^{\circ} 3$ 
THEOREM 1.1. - Assume that

(1) $\Omega \subset \mathbb{R}^{n+1}$ is a $\delta$-Reifenberg flat chord arc domain for some $\delta>0$ small enough;

(2) $\log k_{X} \in \operatorname{VMO}(d \sigma)$.

Then $\Omega$ is a Reifenberg flat domain with vanishing constant.

As mentioned above, under the previous assumptions we conclude also that the harmonic measure is asymptotically optimally doubling (see Definition 1.5 in [18] and Theorem 4.1 in [19]). Hence combining Theorem 1.1 above with Theorems 5.3 or 5.4 in [18] (and taking into account our modified version of $\operatorname{VMO}(d \sigma)$ ) we conclude that the following results hold both for bounded and unbounded domains.

THEOREM 1.2. - Assume that

(1) $\Omega \subset \mathbb{R}^{n+1}$ is a chord arc domain with small enough constant.

(2) $\log k_{X} \in \operatorname{VMO}(\partial \Omega)$.

Then $\Omega$ is a chord arc domain with vanishing constant.

Furthermore when $\Omega$ is an unbounded Reifenberg flat chord arc domain, the harmonic measure with pole at infinity, $\omega$ and $\sigma=\mathcal{H}^{n}\llcorner\partial \Omega$ are mutually absolutely continuous. The Radon-Nikodym theorem ensures that the Poisson kernel with pole at infinity $h(Q)=\frac{d \omega}{d \sigma}(Q) \in$ $L_{\text {loc }}^{1}(d \sigma)$. As before we prove that if $\Omega$ is an unbounded Reifenberg flat chord arc domain, and $\log h \in \operatorname{VMO}(d \sigma)$ then $\Omega$ is a Reifenberg flat domain with vanishing constant.

THEOREM 1.3. - Assume that

(1) $\Omega \subset \mathbb{R}^{n+1}$ is an unbounded $\delta$-Reifenberg flat chord arc domain for some $\delta>0$ small enough;

(2) $\log h \in \operatorname{VMO}(d \sigma)$.

Then $\Omega$ is a Reifenberg flat domain with vanishing constant. Moreover if $h=1 \mathcal{H}^{n}$-a.e. in $\partial \Omega$, then $\Omega$ is a half space.

Combining Theorem 1.3 above with the Main Theorem in [18] (and taking into account our modified version of $\operatorname{VMO}(d \sigma)$ ) we conclude that the following result holds.

THEOREM 1.4. - Assume that

(1) $\Omega \subset \mathbb{R}^{n+1}$ is an unbounded chord arc domain with small enough constant;

(2) $\log h \in \operatorname{VMO}(\partial \Omega)$.

Then $\Omega$ is a chord arc domain with vanishing constant.

A more in depth analysis of the blow-up sequence described in Section 4 allows us to prove that the conjecture stated in [18] holds.

MAIN THEOREM. - Assume that

(1) $\Omega \subset \mathbb{R}^{n+1}$ is a (unbounded) $\delta$-Reifenberg flat chord arc domain for some $\delta>0$ small enough;

(2) $\log k_{X} \in \operatorname{VMO}(d \sigma)(\log h \in \operatorname{VMO}(d \sigma))$.

Then $\Omega$ is a chord arc domain with vanishing constant, i.e., $\vec{n} \in \operatorname{VMO}(d \sigma)$.

Remark. - Note that in [19] we have shown the converse of this, namely that if $\Omega \subset \mathbb{R}^{n+1}$ is a $\delta$-Reifenberg flat chord arc domain and $\vec{n} \in \operatorname{VMO}(d \sigma)$ then $\log k_{X} \in \operatorname{VMO}(d \sigma)$ for every $X \in \Omega$.

Jerison (see [13]) introduced this "end point" problem in higher dimensions, but treated it under more restrictive assumptions, namely that the boundary is given locally as a Lipschitz graph, and the normal derivative data is continuous as opposed to having vanishing mean oscillation. His paper is based on the work of Jerison-Kenig [15] and first points out the 
connection with the work of Alt and Caffarelli [1]. There is an error in Lemma 4 of Jerison's paper. Nevertheless in our previous work (see [18]) we made considerable use of the ideas in [13]. In this paper we bypass this approach. The basic difference between the Main Theorem above and the Main Theorem in [18] (see Section 5) is that in [18] we needed to assume that the harmonic measure was asymptotically optimally doubling and that $\vec{n}$ had small BMO norm. The main ingredient of the proof in [18] was a decay-type argument. The assumption that the BMO norm of $\vec{n}$ was small gave us a starting point for the argument. The main ingredient of the proofs in this paper is a blow-up and hence the assumption on the BMO norm of $\vec{n}$ is not necessary.

It is interesting to compare our results with those of Alt and Caffarelli [1]. In both cases the oscillation of the logarithm of the Poisson kernel controls the geometry (i.e., the "flatness") of the boundary and the oscillation of the unit normal.

THEOREM [1]. - Assume that

(1) $\Omega \subset \mathbb{R}^{n+1}$ is a $\delta$-Reifenberg flat chord arc domain for some $\delta>0$ small enough;

(2) $\log k_{X} \in C^{0, \beta}$ (or $\left.\log h \in C^{0, \beta}\right)$ for some $\beta \in(0,1)$.

Then $\Omega$ is a $C^{1, \alpha}$ domain for some $\alpha \in(0,1)$ which depends on $\beta$ and $n$. Moreover if $\Omega$ is unbounded and $h \equiv 1$ then $\Omega$ is a half-plane.

Jerison showed that $\alpha=\beta$ (see [13]). We would like to emphasize that the hypothesis 1 above is necessary. Keldysh and Lavrentiev (see [17] and [6]) constructed a domain in $\mathbb{R}^{2}$ whose boundary is rectifiable but not Ahlfors regular, whose Poisson kernel is identically equal to 1 and which is not $C^{1}$. Moreover there are examples of domains in $\mathbb{R}^{2}$ whose boundary is Reifenberg flat with vanishing constant, rectifiable but not Ahlfors regular, for which the logarithm of the Poisson kernel is Hölder continuous and which are not even $C^{1}$ domains (see [6]). Furthermore if $n \geqslant 2$ there are examples of chord arc domains satisfying hypothesis 2 , whose boundaries are not $C^{1}$, they contain a neighborhood of the vertex a double cone (see [1] and [18]). These results should also be compared with Pommerenke's theorem [22]:

THEOREM [22]. - Let $\Omega \subset \mathbb{R}^{2}$ be a chord arc domain. Then $\Omega$ is a chord arc domain with vanishing constant if and only if $\log k_{X} \in \operatorname{VMO}(\partial \Omega)$.

We would like to point out that our proofs use a modified version of Alt and Caffarelli's result (see Theorem 2.2 and [20] for a proof).

We now sketch the content of each one of the sections. In Section 2 we prove some technical lemmas which play a central rôle in Sections 3 and 4. These results are of two types: either boundary regularity of non-negative harmonic functions on Reifenberg flat domains, or regularity statements for functions of vanishing mean oscillation. The proofs of Theorem 1.1 and the Main Theorem are accomplished in 2 main stages, described in Sections 3 and 4. In Section 3 we prove gradient bounds for the Green's function in terms of the integral of the corresponding Poisson kernel, provided its logarithm has vanishing mean oscillation. In Section 4 we describe a general construction of a blow up sequence for a Reifenberg flat chord arc domain whose Poisson kernel has logarithm in VMO. In Section 4 we also prove the Main Theorem. The estimates obtained in Section 3 ensure that the limit of this blow up sequence satisfies the hypothesis of Theorem 2.2 (see [20]). Section 4 constitutes the core of this paper. In Appendix A we prove Lemma 3.2 and Rellich's identity for chord arc domains with small constant, verifying a point left open in [18]. In particular in Appendix A we construct an approximation of Reifenberg flat chord arc domains by interior chord arc domains. This is a very useful tool in potential theory.

We finish this introduction by briefly sketching the proof of Theorem 1.1 and Theorem 1.3. This is an application of the blow up technique described in Section 4 . Let $K \subset \mathbb{R}^{n+1}$ be a 
compact set, and let

$$
l=\lim _{r \rightarrow 0} \sup _{Q \in \partial \Omega \cap K} \theta(Q, r) .
$$

Our goal is to show that $l=0$. There exist sequences $\left\{Q_{i}\right\}_{i \geqslant 1} \subset \partial \Omega \cap K$, and $\left\{r_{i}\right\}_{i \geqslant 1} \subset \mathbb{R}$ such that $\lim _{i \rightarrow \infty} Q_{i}=Q_{\infty}, 0<r_{i}, \lim _{i \rightarrow \infty} r_{i}=0$ and

$$
\lim _{i \rightarrow \infty} \theta\left(Q_{i}, r_{i}\right)=l .
$$

We consider the blow up sequences $\Omega_{i}=r_{i}^{-1}\left(\Omega-Q_{i}\right), \partial \Omega_{i}=r_{i}^{-1}\left(\partial \Omega-Q_{i}\right), u_{i}, \omega_{i}$ and $h_{i}$ associated with $Q_{i}$ and $r_{i}$ as described in Section 4 .

Theorem 4.1 ensures that there exists a subsequence (which we relabel) satisfying $\Omega_{i} \rightarrow \Omega_{\infty}$, $\partial \Omega_{i} \rightarrow \partial \Omega_{\infty}$ in the Hausdorff distance sense uniformly on compact sets (see Definition 2.1) and $u_{i} \rightarrow u_{\infty}$ uniformly on compact sets, where $u_{\infty}$ satisfies hypothesis (2.35). Furthermore $\omega_{i} \rightarrow \omega_{\infty}$. Theorems 4.2 and 4.3 guarantee that if $h_{\infty}=\frac{d \omega_{\infty}}{d \sigma_{\infty}}$ then $u_{\infty}$ and $h_{\infty}$ satisfy hypothesis (2.36) and (2.37). Theorem 2.2 allows us to conclude that $\Omega_{\infty}$ is a half plane in $\mathbb{R}^{n+1}$ and $\partial \Omega_{\infty}$ is an $n$-plane. Since $\partial \Omega_{i}$ converges to $\partial \Omega_{\infty}$ in the Hausdorff distance sense uniformly on compact sets and $O \in \partial \Omega_{k}$, for each $k \geqslant 1$, given $\varepsilon>0$ there exists $k_{0} \geqslant 1$ so that for $k \geqslant k_{0}$

$$
D\left[\partial \Omega_{k} \cap B(0,1) ; \partial \Omega_{\infty} \cap B(0,1)\right] \leqslant \varepsilon
$$

Hence

$$
\theta\left(Q_{k}, r_{k}\right) \leqslant \frac{1}{r_{k}} D\left[\partial \Omega \cap B\left(Q_{k}, r_{k}\right) ; L_{k} \cap B\left(Q_{k}, r_{k}\right)\right] \leqslant \varepsilon,
$$

where $L_{k}=\partial \Omega_{\infty}+Q_{k}$ is an $n$-plane through $Q_{k}$. Since by (1.16) $l=\lim _{k \rightarrow \infty} \theta\left(Q_{k}, r_{k}\right)$, we conclude that $l=0$.

\section{Preliminaries}

In this section we prove some technical lemmas that will be useful in the rest of the paper.

DEFINITION 2.1 (Uniform Hausdorff convergence on compact sets). - Given a sequence of closed sets $\left\{A_{i}\right\}_{i}$ in $\mathbb{R}^{n+1}$ we say that $A_{i}$ converges to a closed set $A \subset \mathbb{R}^{n+1}$ (i.e., $A_{i} \rightarrow A$ ) in the Hausdorff distance sense uniformly on compact sets of $\mathbb{R}^{n+1}$ if for any compact set $K \subset \mathbb{R}^{n+1}$ and any $\varepsilon>0$ there exists $i_{0} \geqslant 1$ so that $i \geqslant i_{0}$

$$
\sup \left\{\operatorname{dist}(x, A): x \in A_{i} \cap K\right\}+\sup \left\{\operatorname{dist}\left(x, A_{i}\right): x \in A \cap K\right\} \leqslant \varepsilon .
$$

Given a sequence of open sets $\left\{U_{i}\right\}_{i}$ in $\mathbb{R}^{n+1}$ we say that $U_{i}$ converges to an open set $U \subset \mathbb{R}^{n+1}$ (i.e. $U_{i} \rightarrow U$ ) in the Hausdorff distance sense uniformly on compact sets of $\mathbb{R}^{n+1}$ if $U_{i}^{c} \rightarrow U^{c}$ in the Hausdorff distance sense uniformly on compact sets of $\mathbb{R}^{n+1}$.

For $A, B, C$ closed subsets of $\mathbb{R}^{n+1}$, we use the convention that $\operatorname{dist}(x, B)=+\infty$ when $B=\emptyset$ but $\sup \{\operatorname{dist}(x, A): x \in C\}=0$ when $C=\emptyset$.

DEFINITION 2.2. - Let $\mu$ be a Radon measure on $\mathbb{R}^{n+1}$. We say that $\mu$ is a doubling measure if there exists $C>1$ so that every $Q \in \operatorname{spt} \mu$ and every $r>0$

$$
\mu(B(Q, 2 r)) \leqslant C \mu(B(Q, r)) .
$$

Here spt $\mu$ denotes the support of the measure $\mu$. 
The following lemma gives an improvement of the conclusion of Lemma 4.1 in [14] in the Reifenberg flat case.

LEMMA 2.1. - Given $\varepsilon>0$ there exists $\delta=\delta(n, \varepsilon)>0$ so that if $\Omega$ is a $\delta$-Reifenberg flat domain, then for every $K \subset \mathbb{R}^{n+1}$, there exists $R_{K}>0$ so that if $r \in\left(0, R_{K}\right), Q \in \partial \Omega \cap K$, and $u$ is a non-negative harmonic function in $\Omega \cap B(Q, 4 r)$ which vanishes continuously on $\partial \Omega \cap B(Q, 4 r)$, we have for $X \in B(Q, r) \cap \Omega$

$$
u(X) \leqslant C\left(\frac{|X-Q|}{r}\right)^{1-\varepsilon} \sup _{Y \in \partial B(Q, 2 r) \cap \Omega} u(Y)
$$

where $C$ depends only on $K, n$ and $\varepsilon$.

Proof. - Let $v_{0}$ satisfy $\Delta v_{0}=0$ in $\Omega \cap B(Q, 2 r), v_{0}=1$ on $\partial B(Q, 2 r) \cap \Omega$ and $v_{0}=0$ on $B(Q, 2 r) \cap \partial \Omega$. By the maximum principle for $X \in \Omega \cap B(Q, r)$

$$
u(X) \leqslant\left[\sup _{Y \in \partial B(Q, 2 r) \cap \Omega} u(Y)\right] v_{0}(X) .
$$

Since $\Omega$ is a $\delta$-Reifenberg flat domain Remark 1.1 in [14] holds. Let

$$
\Lambda=\{X=x+t \vec{n}(Q, 2 r) ; x \in L(Q, 2 r) ; t \geqslant-4 \delta r\} .
$$

Let $h_{0}$ satisfy

$$
\begin{cases}\Delta h_{0}=0 & \text { on } \Lambda \cap B(Q, 2 r), \\ h_{0}=0 & \text { on } \partial \Lambda \cap B(Q, 2 r), \\ h_{0}=1 & \text { on } \Lambda \cap \partial B(Q, 2 r) .\end{cases}
$$

By the maximum principle $v_{0}(X) \leqslant h_{0}(X)$ for $X \in \Omega \cap B(Q, 2 r)$. Consider the function $g_{0}$ defined by $g_{0}(x+t \vec{n}(Q, 2 r))=t+4 \delta r ; g_{0}$ is a non-negative harmonic function on $\Lambda \cap B(Q, 2 r), g_{0}=h_{0}=0$ on $\partial \Lambda \cap B(Q, 2 r)$, and therefore by the Comparison principle (Lemma 4.10 in [14]) we have that for $X \in B(Q, r) \cap \Omega$

$$
\frac{h_{0}(X)}{g_{0}(X)} \leqslant C \frac{h_{0}\left(Q+\frac{r}{2} \vec{n}(Q, 2 r)\right)}{r},
$$

and if $X=x+t \vec{n}(Q, 2 r)$

$$
h_{0}(X) \leqslant C \frac{t+4 \delta r}{r} .
$$

Thus for $X \in B(Q, \theta r) \cap \Omega$ with $\theta<1$

$$
v_{0}(X) \leqslant h_{0}(X) \leqslant C(\theta+\delta) .
$$

An iteration process ensures that for $\theta<1$

$$
v_{0}(X) \leqslant[C(\theta+\delta)]^{k} \quad \text { for } X \in B\left(Q, \theta^{k} r\right) \cap \Omega .
$$

In particular

$$
v_{0}(X) \leqslant(2 C \delta)^{k} \quad \text { for } X \in B\left(Q, \delta^{k} r\right) \cap \Omega .
$$

$4^{\mathrm{e}}$ SÉRIE - TOME $36-2003-\mathrm{N}^{\circ} 3$ 
By choosing $\delta>0$ small enough we can ensure that $2 C \delta \leqslant \delta^{1-\varepsilon}$, which implies that

$$
v_{0}(X) \leqslant C\left(\frac{|X-Q|}{r}\right)^{1-\varepsilon} \quad \text { for } X \in B(Q, r) .
$$

Combining (2.4) and (2.12) we obtain (2.3).

Notation. - For $\Omega \in \mathbb{R}^{n+1}$ as above and $X \in \Omega$ we denote by $\delta(X)=\operatorname{dist}(X, \partial \Omega)$.

COROLlaRY 2.1. - Given $\varepsilon>0$ there exists $\delta=\delta(n, \varepsilon)>0$ so that if $\Omega$ is a $\delta$-Reifenberg flat domain then for every $K \subset \mathbb{R}^{n+1}$, there exists $R_{K}>0$ so that if $r \in\left(0, R_{K}\right), Q \in \partial \Omega \cap K$ and $u$ is a non-negative harmonic function in $\Omega \cap B(Q, 4 r)$ which vanishes continuously on $\partial \Omega \cap B(Q, 4 r)$, we have for $X \in B\left(Q, \frac{r}{2}\right) \cap \Omega$

$$
u(X) \leqslant C\left(\frac{\delta(X)}{r}\right)^{1-\varepsilon} \sup _{Y \in \partial B(Q, 4 r) \cap \Omega} u(Y)
$$

where $C$ depends only on $K, n$ and $\varepsilon$.

Proof. - Apply Lemma 2.1 to $\widehat{K}=\left(K, 2 R_{K}\right)=\left\{X \in \mathbb{R}^{n+1}, \operatorname{dist}(X, K) \leqslant 2 R_{K}\right\}$, for $R_{K}$ as above. If $r<\min \left\{R_{K}, 1\right\}, Q \in K \cap \partial \Omega$, and $P \in B(Q, r) \cap \partial \Omega \subset K \cap \partial \Omega$; (2.3) and the maximum principle yield that for $X \in B(P, r) \cap \Omega$

$$
u(X) \leqslant C\left(\frac{|X-P|}{r}\right)^{1-\varepsilon} \sup _{Y \in \partial B(Q, 4 r) \cap \Omega} u(Y),
$$

which implies (2.13).

COROLlaRY 2.2. - Given $\varepsilon>0$ there exists $\delta=\delta(n, \varepsilon)>0$ so that if $\Omega$ is an unbounded Reifenberg flat domain such that

$$
\sup _{Q \in \partial \Omega} \sup _{r>0} \theta(Q, r) \leqslant \delta
$$

and $u$ is a non-negative harmonic function in $\Omega$ which vanishes continuously on $\partial \Omega$, then for $Q \in \partial \Omega, R>0$, and $X \in B(Q, R) \cap \Omega$

$$
u(X) \leqslant C\left(\frac{\delta(X)}{R}\right)^{1-\varepsilon} \sup _{Y \in \partial B(Q, 2 R) \cap \Omega} u(Y),
$$

where $C$ depends only on $n$ and $\varepsilon$.

Proof. - Note that since (2.15) holds for each compact set $K \subset \mathbb{R}^{n+1}$, we can take $R_{K}=\infty$, thus (2.16) follows from (2.13).

COROLLARY 2.3. - Given $\varepsilon>0$ there exists $\delta=\delta(n, \varepsilon)>0$ so that if $\Omega$ is a $\delta$-Reifenberg flat domain, $Q_{0} \in \partial \Omega$, and $u$ is a non-negative harmonic function on $\Omega \cap B\left(Q, 4 R_{0}\right)$ which vanishes continuously on $\partial \Omega \cap B\left(Q, 16 R_{0}\right)$, then for $X \in B\left(Q_{0}, R_{0}\right) \cap \Omega$

$$
u(X) \leqslant C\left(\frac{\delta(X)}{R_{0}}\right)^{1-\varepsilon} \sup _{Y \in \partial B\left(Q_{0}, 16 R_{0}\right) \cap \Omega} u(Y),
$$

where $C$ depends on $R_{0}$, $\varepsilon$ and $n$. 
Proof. - Let $K=\bar{B}\left(Q_{0}, 16 R_{0}\right)$, Corollary 2.1 ensures that there exists $R_{K}>0$ so that for $r_{0}=\frac{1}{2} \min \left\{R_{0}, R_{K}\right\}, Q \in \partial \Omega \cap B\left(Q_{0}, R_{0}\right)$, and $X \in B\left(Q, \frac{r_{0}}{2}\right) \cap \Omega$,

$$
\begin{aligned}
u(X) & \leqslant C\left(\frac{\delta(X)}{r_{0}}\right)^{1-\varepsilon} \sup _{Y \in \partial B\left(Q, 4 R_{0}\right) \cap \Omega} u(Y) \\
& \leqslant C\left(\frac{R_{0}}{r_{0}}\right)^{1-\varepsilon}\left(\frac{\delta(X)}{R_{0}}\right)^{1-\varepsilon} \sup _{Y \in \partial B\left(Q_{0}, 16 R_{0}\right) \cap \Omega} u(Y) .
\end{aligned}
$$

Furthermore by Harnack's principle for $X \in B\left(Q_{0}, R_{0}\right) \cap \Omega$ with $\delta(X) \geqslant \frac{r_{0}}{2}$ we have

$$
u(X) \leqslant C \sup _{Y \in \partial B\left(Q_{0}, 16 R_{0}\right) \cap \Omega} u(Y) \leqslant C\left(\frac{\delta(X)}{r_{0}}\right)^{1-\varepsilon} \sup _{Y \in \partial B\left(Q_{0}, 16 R_{0}\right) \cap \Omega} u(Y) .
$$

Combining (2.18) and (2.19) we obtain (2.17).

The following theorem is a consequence of the John-Nirenberg inequality [16], see Garnett and Jones [10] or [9, Chapter 4] in the Euclidean case. As they point out the result remains true on an Ahlfors regular set. This is not surprising since most of the proof relies on a CalderonZygmund type decomposition, which is possible in this case thanks to the existence of a family of dyadic cubes (see [2] or [5, Chapter 3]).

THEOREM 2.1. - Let $\Omega \subset \mathbb{R}^{n+1}$ be a chord arc domain $f \in \operatorname{VMO}(\partial \Omega)$ and $h=e^{f}$ then for all $Q \in \partial \Omega, r \in(0, \operatorname{diam} \Omega)$ and $q<\infty$

$$
\begin{gathered}
\left(\oint_{B(Q, r)} h^{q} d \sigma\right)^{\frac{1}{q}} \leqslant C_{q} \underset{B(Q, r)}{\oint_{B(Q, r)} h d \sigma} \\
\left(\oint_{B(Q, r)} h^{-q} d \sigma\right)^{\frac{1}{q}} \leqslant C_{q} \oint_{B(Q, r)} h^{-1} d \sigma .
\end{gathered}
$$

Here $C_{q}$ only depends on the VMO character of $f$, on $n$, $q$ and the Ahlfors constant for $\sigma$.

Proof. - Since $f \in \operatorname{VMO}(\partial \Omega)$, then $f \in \operatorname{BMO}(\partial \Omega)$ and there exits $p>1$ such that $h$, $h^{-1} \in A_{p}$. Since $\operatorname{VMO}(\partial \Omega)$ is the closure of the class of bounded uniformly continuous functions in $\mathrm{BMO}(\partial \Omega)$ in $\partial \Omega$, then

$$
\operatorname{dist}\left(f, L^{\infty}\right)=\inf _{g \in L^{\infty}}\left\{\|f-g\|_{*}\right\}=0
$$

where $\|\cdot\|_{*}$ denotes the norm in $\operatorname{BMO}(\partial \Omega)$ see Definition 1.12. Combining Corollary 1.1, and Lemma 1.4 in [10] we conclude that $h, h^{-1} \in A_{q}$ for every $q>1$.

COROLlary 2.4. - Let $\Omega \subset \mathbb{R}^{n+1}$ be a chord arc domain and $\log h \in \mathrm{VMO}(\partial \Omega)$, then for all $\varepsilon>0, Q \in \partial \Omega, r \in(0, \operatorname{diam} \Omega)$, and $E \subset B(Q, r) \cap \partial \Omega$

$$
C_{\varepsilon}^{-1}\left(\frac{\sigma(E)}{\sigma(B(Q, r))}\right)^{1+\varepsilon} \leqslant \frac{\omega(E)}{\omega(B(Q, r))} \leqslant C_{\varepsilon}\left(\frac{\sigma(E)}{\sigma(B(Q, r))}\right)^{1-\varepsilon},
$$

where $\omega(A)=\int_{A} h d \sigma$. Here $C_{\varepsilon}$ only depends on $n, \varepsilon$ and the Ahlfors constant of $\sigma$. 
Proof. - Let $q=\frac{1}{\varepsilon}$. For $E \subset \partial \Omega \cap B(Q, r)$, applying (2.20) we have

$$
\begin{aligned}
\omega(E) & \leqslant \int_{E} h d \sigma \leqslant\left(\int_{B(Q, r)} h^{q} d \sigma\right)^{\frac{1}{q}}\left(\int_{E} d \sigma\right)^{1-\frac{1}{q}} \\
& \leqslant \sigma(B(Q, r))^{\frac{1}{q}}\left(\oint_{B(Q, r)} h^{q} d \sigma\right)^{\frac{1}{q}} \sigma(E)^{1-\frac{1}{q}} \\
& \leqslant C_{q} \sigma(B(Q, r))^{\frac{1}{q}}\left(\oint_{B(Q, r)} h d \sigma\right) \sigma(E)^{1-\frac{1}{q}} \\
& \leqslant C_{\varepsilon}\left(\frac{\sigma(E)}{\sigma(B(Q, r))}\right)^{1-\varepsilon} \omega(B(Q, r))
\end{aligned}
$$

which shows that

$$
\frac{\omega(E)}{\omega(B(Q, r))} \leqslant C_{\varepsilon}\left(\frac{\sigma(E)}{\sigma(B(Q, r))}\right)^{1-\varepsilon} .
$$

Since $\sigma(A)=\int_{A} h^{-1} d \omega$, the argument above applied to $h^{-1}$ rather than $h$, yields the first inequality in (2.23).

Let us finish this section by specifying our set up. Let $\Omega \subset \mathbb{R}^{n+1}$ be a $\delta$-Reifenberg flat chord arc domain ( $\delta>0$ is chosen so that $\Omega$ is an NTA domain, see [19]). Let $A \subset \Omega$ be fixed, and let $u$ denote the Green's function of $\Omega$ with either pole at infinity (see [18, Lemma 3.7]) or the Green's function of $\Omega$ with pole at $A$. By the results of [25] or [4] we know that $\omega$ and $\omega^{A}$ the harmonic measures of $\Omega$ with pole at infinity and pole at $A$ respectively are $A_{\infty}$-weights with respect to $\sigma$, the surface measure to the boundary. Let $k_{A}=\frac{d \omega^{A}}{d \sigma}$ denote the Poisson kernel with pole at $A$ and $h=\frac{d \omega}{d \sigma}$ denote the Poisson kernel with pole at infinity. Recall that if $u$ denotes the Green's function with pole at infinity we have

$$
\begin{cases}\Delta u=0 & \text { in } \Omega \\ u=0 & \text { on } \partial \Omega \\ u>0 & \text { in } \Omega\end{cases}
$$

and

$$
\int_{\Omega} u \Delta \varphi=\int_{\partial \Omega} \varphi d \omega=\int_{\partial \Omega} \varphi h d \mathcal{H}^{n} \quad \text { for all } \varphi \in C_{c}^{\infty}\left(\mathbb{R}^{n+1}\right) .
$$

Similarly note that if $u$ denotes the Green's function with pole at $A$ then we have

$$
\begin{cases}\Delta u=0 & \text { in } \Omega \cap B(Q, R) \\ u=0 & \text { on } \partial \Omega \cap B(Q, R) \\ u>0 & \text { on } \Omega \cap B(Q, R)\end{cases}
$$

and

$$
\int_{\Omega} u \Delta \varphi=\int_{\partial \Omega} \varphi d \omega^{A}=\int_{\partial \Omega} \varphi k_{A} d \mathcal{H}^{n} \quad \text { for all } \varphi \in C_{C}^{\infty}(B(Q, R))
$$


for any $Q \in \partial \Omega$ and $R>0$ so that $A \notin B(Q, R)$. In order to unify our presentation we denote by $\omega$ the harmonic measure with either finite or infinite pole, and by $h$ the corresponding Poisson kernel.

The following 2 lemmas are used in the proof of Lemma 4.2. We present them here to avoid interrupting the flow of ideas in Section 4. The first lemma is essentially Lemma 5.4 in [18].

LEMMA 2.2. - Let $\Omega \subset \mathbb{R}^{n+1}$ be a $\delta$-Reifenberg flat chord arc domain. Let $X \in \Omega$ then for $\mathcal{H}^{n}$ a.e. $Q \in \partial \Omega$

$$
\frac{d \omega^{X}(Q)}{d \omega}=\frac{k_{X}(Q)}{h(Q)}=\lim _{X \rightarrow 0} \frac{\omega^{X}(B(Q, r))}{\omega(B(Q, r))}=\lim _{Z \rightarrow Q} \frac{G(X, Z)}{u(Z)} .
$$

Here $\omega^{X}$ denotes the harmonic measure, $G(X,-)$ denotes the Green's function, and $k_{X}$ the Poisson kernel for $\Omega$ with pole at $X$. Let $K(X, Q)=\frac{k_{X}(Q)}{h(Q)}$. There exist constants $C>1, N_{0}>1$ and $\alpha \in(0,1)$ so that for $s \in(0, \operatorname{diam} \Omega)$, and $Q_{0} \in \partial \Omega$, if $X \in \Omega \backslash B\left(Q_{0}, 2 N_{0} s\right)$, then for every $P, Q \in B\left(Q_{0}, s\right) \cap \partial \Omega$

$$
|K(X, Q)-K(X, P)| \leqslant C K(X, Q)\left(\frac{|Q-P|}{s}\right)^{\alpha} .
$$

Although the hypothesis above are somewhat weaker than those in the statement of Lemma 5.4 in [18], the reader will easily check that the proof presented in [18] works in this setting. Simply note that $\omega^{X}, \omega$ and $\sigma$ are doubling measures on $\partial \Omega$ and $\omega^{X}, \omega \in A_{\infty}(d \sigma)$. Thus $\omega^{X}$ and $\omega$ are mutually absolutely continuous, and the proof presented in [18] goes through.

Lemma 2.3. - Let $\Omega \subset \mathbb{R}^{n+1}$ be a $\delta$-Reifenberg flat chord arc domain. Assume that $h$ the Poisson kernel satisfies for all $Q \in \partial \Omega$, and $r \in(0, \operatorname{diam} \Omega)$

$$
\left(\oint_{B(Q, r)} h^{2} d \sigma\right)^{\frac{1}{2}} \leqslant C_{0} \oint_{B(Q, r)} h d \sigma .
$$

There exist constants $C>1$ and $N_{0}>1$ so that for $r \in(0$, diam $\Omega)$, and $Q \in \partial \Omega$ if $X \in \Omega \backslash B\left(Q, 2 N_{0} r\right)$ then

$$
\left(\oint_{B(Q, r)} k_{X}^{2} d \sigma\right)^{\frac{1}{2}} \leqslant C \oint_{B(Q, r)} k_{X} d \sigma .
$$

Proof. - Let $N_{0}>1$ be as in Lemma 2.2. Let $Q \in \partial \Omega, r \in(0, \operatorname{diam} \Omega)$ and $X \in \Omega \backslash B(Q, 2 N r)$, then using (2.31) and (2.32) we have

$$
\begin{aligned}
& \left(\int_{B(Q, r)} k_{X}^{2}(P) d \sigma(P)\right)^{\frac{1}{2}} \\
& =\left(\oint_{B(Q, r)} \frac{k_{X}^{2}(P)}{h^{2}(P)} h^{2}(P) d \sigma(P)\right)^{\frac{1}{2}} \leqslant C K(X, Q)\left(f_{B(Q, r)} h^{2}(P) d \sigma(P)\right)^{\frac{1}{2}} \\
& \leqslant C K(X, Q) \underset{B(Q, r)}{f_{(P)} h(P) d \sigma(P)}
\end{aligned}
$$




$$
\begin{aligned}
& \leqslant C \underset{B(Q, r)}{\oint_{B(Q, r)} h(P)[K(X, Q)-K(X, P)] d \sigma(P)+C f_{B(P, r)} h(P) K(X, P) d \sigma(P)} \\
& \leqslant C \oint_{B(Q, r)} h(P) K(X, P) d \sigma(P) \leqslant C f_{B(Q, r)} k_{X}(P) d \sigma(P) .
\end{aligned}
$$

We finish this section with the statement of a theorem that plays a crucial role in our proof. It generalizes some of the results that appear in [1]. In Sections 7 and 8 of [1], Alt and Caffarelli prove that if $\Omega$ is a Reifenberg flat chord arc domain and $\log h \in C^{0, \beta}(\partial \Omega)$ for some $\beta \in(0,1)$ then $\Omega$ is a $C^{1, \alpha}$ domain for some $\alpha \in(0,1)$. In particular they show that if $h \equiv 1$ then $\Omega$ is a half space.

THEOREM 2.2. - There exists $\delta_{n}>0$ so that if $\Omega \subset \mathbb{R}^{n+1}$ is an unbounded $\delta$-Reifenberg flat chord arc domain $\left(\right.$ for $\left.\delta \in\left(0, \delta_{n}\right)\right)$ and $v$ and $k$ satisfy

$$
\begin{cases}\Delta v=0 & \text { in } \Omega \\ v>0 & \text { in } \Omega \\ v=0 & \text { on } \partial \Omega\end{cases}
$$

and

$$
\int_{\Omega} v \Delta \varphi=\int_{\partial \Omega} \varphi k d \mathcal{H}^{n} \quad \text { for all } \varphi \in C_{c}^{\infty} \mathbb{R}^{n+1}
$$

with

$$
\sup _{X \in \Omega}|\nabla v(X)| \leqslant 1 \quad \text { and } \quad k(Q) \geqslant 1 \quad \text { for } \mathcal{H}^{n} \text { a.e. } Q \in \partial \Omega,
$$

then $\Omega$ is a half space, and in suitable coordinates $v\left(x, x_{n+1}\right)=x_{n+1}$.

Note that the uniqueness (modulo multiplication by a positive constant) of the Green's function with pole at infinity for unbounded NTA domains allows us to conclude that $k=1$ on $\partial \Omega$ (see [19]). The proof of Theorem 2.2 follows the same steps as the argument presented in Sections 7 and 8 of [1], for a proof see [20].

\section{Gradient bound for the Green's function}

As mentioned in the introduction the proofs of our results are done in 2 stages. First we give a bound for the gradient of the Green's function in terms of the integral of the Poisson kernel. Second we use this estimate to produce a blow up sequence whose limit satisfies the hypothesis of Alt and Caffarelli's result as stated in Theorem 2.2. In this section we prove the gradient estimate.

From now on we assume that $\Omega \subset \mathbb{R}^{n+1}$ is a $\delta$-Reifenberg flat chord arc domain, where $\delta>0$ is chosen so that, in the unbounded case Corollaries 2.2, and 2.3 hold for $\varepsilon=\frac{1}{4}$ and in the bounded case Corollary 2.3 holds for $\varepsilon=\frac{1}{4}$. Moreover we assume that $\log h \in \operatorname{VMO}(\partial \Omega)$. This hypothesis ensures that $h \in L_{\mathrm{loc}}^{2}(d \sigma)$ and that for $Q \in \partial \Omega, r \in(0, \operatorname{diam} \Omega)$, and $s \in(0, r)$

$$
\left(\oint_{B(Q, r)} h^{2} d \sigma\right)^{\frac{1}{2}} \leqslant C \oint_{B(Q, r)} h d \sigma
$$




$$
C^{-1}\left(\frac{\sigma(B(Q, s))}{\sigma(B(Q, r))}\right)^{1+\frac{1}{8 n}} \leqslant \frac{\omega(B(Q, s))}{\omega(B(Q, r))} \leqslant C\left(\frac{\sigma(B(Q, s))}{\sigma(B(Q, r))}\right)^{1-\frac{1}{8 n}},
$$

where $C$ is a constant that only depends on $n$, and the Ahlfors constant of $\sigma$.

Recall that $u$ denotes either the Green's function with pôle $A \in \Omega$ or with pôle at infinity (if $\Omega$ is unbounded); $h$ denotes the corresponding Poisson kernel and $\omega$ the associated harmonic measure $d \omega=h d \sigma$. We denote by $\ell$ one quarter of the distance from the pôle of $u$ to $\partial \Omega$, i.e., $\ell=\delta(A) / 4$ or $\ell=+\infty$.

THEOREM 3.1. - Let $\Omega \subset \mathbb{R}^{n+1}$ be a $\delta$-Reifenberg flat chord arc domain satisfying Corollaries 2.2 and 2.3 with $\varepsilon=\frac{1}{4}$. Let $u$ denote the Green's function with pôle at infinity, $\omega$ the harmonic measure with pôle at infinity, and $h=\frac{d \omega}{d \sigma}$ the corresponding Poisson kernel. Assume that $\log h \in \operatorname{VMO}(\partial \Omega)$, then for all $X \in \Omega$ we have

$$
|\nabla u(X)| \leqslant \int_{\partial \Omega} h(Q) d \omega^{X}(Q) .
$$

THEOREM 3.2. - Let $\Omega \subset \mathbb{R}^{n+1}$ be a $\delta$-Reifenberg flat chord arc domain satisfying Corollary 2.3 with $\varepsilon=\frac{1}{4}$. Let $G(A,-)$ denote the Green's function with pole at $A$ and $k_{A}=\frac{d \omega^{A}}{d \sigma}$ the corresponding Poisson kernel. Assume that $\log k_{A} \in \operatorname{VMO}(\partial \Omega)$, then for all $X \in \Omega \cap\left\{Y \in \mathbb{R}^{n+1}: \delta(Y)<\delta(A) / 8\right\}$ we have

$$
|\nabla G(A, X)| \leqslant \int_{\partial \Omega} k_{A}(Q) d \omega^{X}+C \frac{1}{\delta(A)^{n}}\left(\frac{\delta(X)}{\delta(A)}\right)^{\frac{3}{4}} \omega^{A}\left(B\left(Q_{X}, \delta(A)\right)\right),
$$

for any $Q_{X} \in \partial \Omega$ such that $X \in B\left(Q_{X}, \delta(A) / 8\right) \cap \Omega$.

LEMMA 3.1. - Let $X_{*} \in \Omega$. Let $u, \omega$ and $h$ as above, and assume that $h \in L_{\text {loc }}^{2}(d \sigma)$. Then for $\omega$ a.e. $Q \in \partial \Omega, \nabla u(X)$ converges non-tangentially to $F(Q)$, and $F \in L_{\mathrm{loc}}^{1}\left(d \omega^{X_{*}}\right)$.

Proof. - Let $l=\min \{1, \ell\}$. Let $K \subset \mathbb{R}^{n+1}$ be a compact set, let

$$
\widehat{K}=\left\{X \in \mathbb{R}^{n+1}: \operatorname{dist}(X, K) \leqslant l\right\} .
$$

Let $Q \in \widehat{K} \cap \partial \Omega$, and $X \in \Gamma(Q)$ with $\delta(X)<\ell$. Here $\Gamma(Q)$ denotes a nontangential access region. By a standard estimate for non-negative harmonic functions we have

$$
|\nabla u(X)| \leqslant C \frac{u(X)}{\delta(X)}
$$

Furthermore by Lemma 4.8 in [14] there is $C>1$ so that for every $Q \in \widehat{K} \cap \partial \Omega, X \in \Gamma(Q)$ with $\delta(X)<\ell$, if $Y \in \Omega \backslash B(Q, 2 \delta(X))$

$$
C^{-1}<\frac{\omega^{Y}(B(Q, \delta(X)))}{\delta(X)^{n-1} G(X, Y)}<C .
$$

Since $A \in \Omega \backslash B(Q, 2 \delta(X))$ for $X \in \Omega$ with $\delta(X)<\ell$, (3.6) yields

$$
C^{-1}<\frac{\omega^{A}(B(Q, \delta(X)))}{\delta(X)^{n-1} G(X, A)}<C .
$$

$4^{\mathrm{e}}$ SÉRIE - TOME $36-2003-\mathrm{N}^{\circ} 3$ 
By the construction described in the proof of Lemma 3.7 in [18], we know that letting $|Y|$ tend to infinity for $Q \in \widehat{K} \cap \partial \Omega$ and $X \in \Gamma(Q)$, (3.6) yields

$$
C^{-1}<\frac{\omega(B(Q, \delta(X)))}{\delta(X)^{n-1} u(X)}<C
$$

Combining (3.5), (3.7) and (3.8) we have that for $X \in \Gamma(Q)$ with $\delta(X) \leqslant l$,

$$
|\nabla u(X)| \leqslant C \frac{u(X)}{\delta(X)} \leqslant C \frac{1}{\delta(X)^{n}} \int_{\partial \Omega \cap B(Q, \delta(X))} h d \sigma,
$$

so that if $\delta(X) \leqslant l$

$$
\sup _{\substack{X \in \Gamma(Q) \\ \delta(X) \leqslant l}}|\nabla u(X)| \leqslant C M_{l}(h)(Q)
$$

where

$$
M_{l}(h)(Q)=\sup _{0<r \leqslant l} \frac{1}{r^{n}} \int_{B(Q, r) \cap \partial \Omega} h d \sigma .
$$

Since

$$
\int_{K}\left[M_{l}(h)\right]^{2} d \sigma \leqslant C \int_{\widehat{K}} h^{2} d \sigma<\infty,
$$

we see that the truncated non-tangential maximal function of $\nabla u$ is in $L_{\text {loc }}^{2}(d \sigma)$ and hence in $L_{\text {loc }}^{1}\left(d \omega^{X_{*}}\right)$. By Fatou's theorem for NTA domains (see [14] Theorem 5.8 and Lemma 8.3 as well as Lemma 3.3 in Appendix A) we know that $\nabla u$ converges non-tangentially to $F$, and $F \in L_{\mathrm{loc}}^{1}\left(d \omega^{X_{*}}\right)$.

Lemma 3.2. - Let $F$ be the non-tangential limit of $\nabla u$. Then since $h \in L_{\text {loc }}^{2}(d \sigma)$, for $\mathcal{H}^{n}$ a.e. $Q \in \partial \Omega$ we have that

$$
F(Q)=h(Q) \vec{n}(Q) .
$$

The proof of this lemma appears in Sections A.1 and A.2 of Appendix A.

LEMMA 3.3. - Let $\Omega \subset \mathbb{R}^{n+1}$ be an unbounded $\delta$-Reifenberg flat chord arc domain satisfying Corollaries 2.2 and 2.3 with $\varepsilon=\frac{1}{4}$. Assume that $\log h \in \operatorname{VMO}(\partial \Omega)$, and that $0 \in \partial \Omega$. Fix $R>1$ large and let $\varphi_{R} \in C_{c}^{\infty}\left(\mathbb{R}^{n+1}\right), \varphi_{R} \equiv 1$ for $|X| \leqslant R$, spt $\varphi_{R} \subset B(0,2 R), 0 \leqslant \varphi_{R} \leqslant 1$ and $\left|\nabla \varphi_{R}\right| \leqslant C / R,\left|\Delta \varphi_{R}\right| \leqslant C / R^{2}$. For $X \in \Omega$ define

$$
\omega_{R}(X)=\int_{\Omega} G(X, Y) \Delta\left[\varphi_{R}(Y) \nabla u(Y)\right] d Y,
$$

where $u$ denotes the Green's function of $\Omega$ with pole at $\infty$. Then $\left.\omega_{R}\right|_{\partial \Omega} \equiv 0, \omega_{R} \in C^{\alpha}(\bar{\Omega})$ for some $\alpha \in(0,1)$, and we have the following estimates for $X \in \Omega$

$$
\left|\omega_{R}(X)\right| \leqslant C \frac{\delta(X)^{3 / 4}}{R^{1 / 2}} \text { for }|X|<\frac{R}{2} .
$$




$$
\begin{aligned}
& \left|\omega_{R}(X)\right| \leqslant C R^{n}\left[\frac{\omega(B(0, R))}{R^{n}}\right]^{2}\left(\frac{\delta(X)}{|X|}\right)^{\frac{3}{4}} \frac{1}{\omega(B(0,|X|))} \text { for }|X| \geqslant 4 R . \\
& \left|\omega_{R}(X)\right| \leqslant C\left(\frac{\delta(X)}{R}\right)^{\frac{3}{4}} \frac{\omega(B(0, R))}{R^{n}} \text { for } \frac{R}{2} \leqslant|X| \leqslant 4 R .
\end{aligned}
$$

Proof. - Let $V(X)=\nabla u(X)$ for $X \in \Omega$. Then $\Delta\left(\varphi_{R} V\right)=\left(\Delta \varphi_{R}\right) V+2 \nabla \varphi_{R} \cdot \nabla V$ so that

$$
\omega_{R}(X)=\omega_{R}^{1}(X)+\omega_{R}^{2}(X)
$$

with

$$
\omega_{R}^{1}(X)=\int_{\Omega} G(X, Y) \Delta \varphi_{R}(Y) V(Y) d Y,
$$

and

$$
\omega_{R}^{2}(X)=2 \int_{\Omega} G(X, Y) \nabla \varphi_{R}(Y) \cdot \nabla V(Y) d Y .
$$

Note that

$$
|V(Y)| \leqslant C \frac{u(Y)}{\delta(Y)} \quad \text { and } \quad|\nabla V(Y)| \leqslant C \frac{u(Y)}{\delta^{2}(Y)},
$$

also spt $\nabla \varphi_{R}$, spt $\Delta \varphi_{R} \subset\{R<|Y|<2 R\}$. Let

$$
I_{R}=\int_{\{R<|Y|<2 R\} \cap \Omega}\left(\frac{u(Y)}{\delta(Y)}\right)^{2} d Y .
$$

CLAIM. - If $\Omega$ is as above then

$$
I_{R} \leqslant C R^{n+1} \frac{u^{2}\left(A_{2 R}\right)}{R^{2}} .
$$

In fact note that by Harnack's principle and our assumption that $\delta$ is chosen so that Corollary 2.2 holds for $\varepsilon=\frac{1}{4}$ we have that for $Y \in \Omega \cap B(0,2 R) \backslash B(0, R)$

$$
u(Y) \leqslant C\left(\frac{\delta(Y)}{R}\right)^{\frac{3}{4}} u\left(A_{2 R}\right) .
$$

Thus

$$
I_{R} \leqslant C u^{2}\left(A_{2 R}\right)\left[\frac{1}{R^{3 / 2}} \int_{\{R<|Y|<2 R\} \cap \Omega} \frac{d Y}{\delta(Y)^{1 / 2}}\right] .
$$

We want to show that the term in brackets is bounded above by $\frac{C}{R^{2}} R^{n+1}$. Scaling shows that it is enough to prove this for $R=1$, i.e., we have to show that for $\Omega$ as in Corollaries 2.2 and 2.3

$$
\int_{\{1<|Y|<2\} \cap \Omega} \frac{d Y}{\delta(Y)^{1 / 2}} \leqslant C .
$$


Let $j \geqslant 0$, and

$$
A_{j}=\Omega \cap\left\{|Y|<2: 2^{-j}<\delta(Y) \leqslant 2^{-j+1}\right\} .
$$

Cover $\partial \Omega \cap B(0,2)$ by balls $\left\{B\left(Q_{i}, 1 / 2^{j-1}\right)\right\}_{i=1}^{N}$ centered in $\partial \Omega$ and so that $\left|Q_{i}-Q_{l}\right| \geqslant 1 / 2^{j}$ for $i \neq l$. Since $\partial \Omega$ is Ahlfors regular, it is straightforward that $N \leqslant C 2^{j n}$, where $C$ depends on $n$ and on the Ahlfors regularity constant of $\partial \Omega$. If $Y \in A_{j}$ there exists $X \in \partial \Omega$ so that $|X-Y| \leqslant 1 / 2^{j-1}$ and $Q_{i} \in \partial \Omega$ so that $\left|Q_{i}-Y\right| \leqslant 1 / 2^{j-2}$. On the other hand since $\delta(Y)>1 / 2^{j},\left|Q_{i}-Y\right|>1 / 2^{j}$. Thus $\left\{B\left(Q_{i}, 1 / 2^{j-2}\right) \backslash B\left(Q_{i}, 1 / 2^{j}\right)\right\}_{i=1}^{N}$ covers $A_{j}$ and

$$
\mathcal{H}^{n+1}\left(A_{j}\right) \leqslant C_{n} 2^{n j}\left\{\left(\frac{1}{2^{j-2}}\right)^{n+1}-\left(\frac{1}{2^{j}}\right)^{n+1}\right\} \leqslant C_{n} 2^{-j},
$$

which implies that

$$
\int_{\{1<|Y|<2\} \cap \Omega} \frac{d Y}{\delta(Y)^{1 / 2}}=\sum_{j=0}^{\infty} \int_{A_{j}} \frac{d Y}{\delta(Y)^{1 / 2}} \leqslant C_{n} \sum_{j=0}^{\infty} 2^{-j / 2} \leqslant C,
$$

which proves the claim.

Case 1. Let $|X| \leqslant \frac{R}{2}$. Then

$$
\left|\omega_{R}^{2}(X)\right| \leqslant \frac{C}{R} \int_{\{R<|Y|<2 R\} \cap \Omega} G(X, Y) \frac{u(Y)}{\delta(Y)^{2}} d Y .
$$

Let $A_{S}=A(0, S / 2)$; i.e., $S / M \leqslant\left|A_{S}\right| \leqslant S$ and $\delta\left(A_{S}\right) \geqslant S / M$ (see Definition 3.1 of NTA domain in [18]). Then for $Y \in \Omega \cap B(0,2 R) \backslash \overline{B(0, R)}$ we have, using Corollary 2.3, that

$$
G(X, Y) \leqslant C\left[\frac{\delta(X)}{R}\right]^{\frac{3}{4}} G\left(A_{R}, Y\right) .
$$

Moreover by the Comparison Principle (Lemma 4.10 in [14]) we have that for $Y \in\{R<|Y|<2 R\}$

$$
\frac{G\left(A_{R}, Y\right)}{G\left(A_{R}, A_{2 R}\right)} \leqslant C \frac{u(Y)}{u\left(A_{2 R}\right)}
$$

hence

$$
G\left(A_{R}, Y\right) \leqslant C G\left(A_{R}, A_{2 R}\right) \frac{u(Y)}{u\left(A_{2 R}\right)} \leqslant \frac{C}{R^{n-1}} \frac{u(Y)}{u\left(A_{2 R}\right)},
$$

and combining (3.30), (3.33), (3.23), (3.8), (3.2), and using the fact that $R>1$ we have that

$$
\begin{aligned}
\left|\omega_{R}^{2}(X)\right| & \leqslant \frac{C}{R^{n}}\left(\frac{\delta(X)}{R}\right)^{\frac{3}{4}} \frac{1}{u\left(A_{2 R}\right)} \int_{\{R<|Y|<2 R\} \cap \Omega} \frac{u^{2}(Y)}{\delta(Y)^{2}} d Y \\
& \leqslant \frac{C}{R^{n}}\left(\frac{\delta(X)}{R}\right)^{\frac{3}{4}} \frac{1}{u\left(A_{2 R}\right)} I_{R} \\
& \leqslant C\left(\frac{\delta(X)}{R}\right)^{\frac{3}{4}} \frac{u\left(A_{2 R}\right)}{R} \leqslant C\left(\frac{\delta(X)}{R}\right)^{\frac{3}{4}} \frac{\omega(B(0,2 R))}{R^{n}}
\end{aligned}
$$




$$
\leqslant C\left(\frac{\delta(X)}{R}\right)^{\frac{3}{4}} \frac{\omega(B(0, R))}{\sigma(B(0, R))} \leqslant C\left(\frac{\delta(X)}{R}\right)^{\frac{3}{4}} R^{\frac{1}{4}} \omega(B(0,1)) .
$$

Hence for $|X| \leqslant \frac{R}{2}$

$$
\left|\omega_{R}^{2}(X)\right| \leqslant C \frac{\delta(X)^{3 / 4}}{R^{1 / 2}}
$$

We now estimate the term $\omega_{R}^{1}(X)$. Using (3.21), (3.31) and (3.33) we obtain

$$
\begin{aligned}
\left|\omega_{R}^{1}(X)\right| & \leqslant \int_{\Omega} G(X, Y)\left|\Delta \varphi_{R}(Y)\right||V(Y)| d Y \\
& \leqslant C\left(\frac{\delta(X)}{R}\right)^{\frac{3}{4}} \frac{1}{R^{2}} \int_{\Omega \cap\{R<|Y|<2 R\}} G\left(A_{R}, Y\right) \frac{u(Y)}{\delta(Y)} d Y \\
& \leqslant C \frac{1}{R^{n+1}}\left(\frac{\delta(X)}{R}\right)^{\frac{3}{4}} \int_{\Omega \cap\{R<|Y|<2 R\}} \frac{u(Y)^{2}}{\delta(Y)} \frac{d Y}{u\left(A_{2 R}\right)} .
\end{aligned}
$$

Since for $Y \in \Omega$ with $R<|Y|<2 R, \delta(Y) \leqslant 2 R$, (3.37) becomes

$$
\begin{aligned}
\left|\omega_{R}^{1}(X)\right| & \leqslant \frac{C}{R^{n}} \frac{1}{u\left(A_{2 R}\right)}\left(\frac{\delta(X)}{R}\right)^{\frac{3}{4}} \int_{\Omega \cap\{R<|Y|<2 R\}}\left(\frac{u(Y)}{\delta(Y)}\right)^{2} d Y \\
& \leqslant C \frac{u\left(A_{2 R}\right)}{R}\left(\frac{\delta(X)}{R}\right)^{\frac{3}{4}} \leqslant C \frac{\delta(X)^{\frac{3}{4}}}{R^{\frac{1}{2}}},
\end{aligned}
$$

because of (3.23) and (3.34). This concludes the proof of (3.15).

Case 2. Let $|X| \geqslant 4 R$. Assume that $2^{j} R \leqslant|X|<2^{j+1} R$ for some $j \geqslant 2$. Let $A_{j}=A\left(0,2^{j} R\right)$ be a non-tangential point for 0 at radius $2^{j} R$. For $Y \in \Omega$ with $R<|Y|<2 R$, by Corollary 2.3 the comparison principle and (3.8) we have

$$
\begin{aligned}
G(X, Y) & \leqslant C\left(\frac{\delta(X)}{2^{j} R}\right)^{\frac{3}{4}} G\left(A_{j}, Y\right) \leqslant C\left(\frac{\delta(X)}{2^{j} R}\right)^{\frac{3}{4}} G\left(A_{j}, A_{R}\right) \frac{u(Y)}{u\left(A_{R}\right)} \\
& \leqslant C\left(\frac{\delta(X)}{2^{j} R}\right)^{\frac{3}{4}} G\left(A_{j}, A_{j-1}\right) \frac{u(Y)}{u\left(A_{j-1}\right)} \leqslant C\left(\frac{\delta(X)}{2^{j} R}\right)^{\frac{3}{4}} \frac{1}{\left(2^{j-1} R\right)^{n-1}} \frac{u(Y)}{u\left(A_{j-1}\right)} \\
& \leqslant C\left(\frac{\delta(X)}{2^{j} R}\right)^{\frac{3}{4}} \frac{u(Y)}{\omega\left(B\left(0,2^{j-1} R\right)\right)} \leqslant C\left(\frac{\delta(X)}{2^{j} R}\right)^{\frac{3}{4}} \frac{u(Y)}{\omega\left(B\left(0,2^{j} R\right)\right)} .
\end{aligned}
$$

Thus using (3.23), the fact that $\omega$ is a doubling measure, and (3.8) we have

$$
\begin{aligned}
\left|\omega_{R}^{2}(X)\right| & \leqslant \frac{C}{R} \int_{\{R<|Y|<2 R\} \cap \Omega} G(X, Y) \frac{u(Y)}{\delta(Y)^{2}} d Y \\
& \leqslant \frac{C}{R}\left(\frac{\delta(X)}{2^{j} R}\right)^{\frac{3}{4}} \frac{1}{\omega\left(B\left(0,2^{j} R\right)\right)} \int_{\{R<|Y|<2 R\} \cap \Omega} \frac{u^{2}(Y)}{\delta(Y)^{2}} d Y \\
& \leqslant \frac{C}{R}\left(\frac{\delta(X)}{2^{j} R}\right)^{\frac{3}{4}} \frac{I_{R}}{\omega\left(B\left(0,2^{j} R\right)\right)}
\end{aligned}
$$




$$
\begin{aligned}
& \leqslant \frac{C R^{n} u^{2}\left(A_{R}\right)}{R^{2}}\left(\frac{\delta(X)}{2^{j} R}\right)^{\frac{3}{4}} \frac{1}{\omega(B(0,|X|))} \\
& \leqslant C R^{n-2}\left(\frac{\omega(B(0, R))}{R^{n-1}}\right)^{2}\left(\frac{\delta(X)}{|X|}\right)^{\frac{3}{4}} \frac{1}{\omega(B(0,|X|))} \\
& \leqslant C R^{n}\left[\frac{\omega(B(0, R))}{R^{n}}\right]^{2}\left(\frac{\delta(X)}{|X|}\right)^{\frac{3}{4}} \frac{1}{\omega(B(0,|X|))} .
\end{aligned}
$$

In order to finish the proof of (3.16) we need to estimate $\omega_{R}^{1}(X)$ for $|X| \geqslant 4 R$. By (3.21), (3.39) and the computation in (3.40) we obtain

$$
\begin{aligned}
\left|\omega_{R}^{1}(X)\right| & \leqslant \frac{C}{R^{2}}\left(\frac{\delta(X)}{2^{j} R}\right)^{\frac{3}{4}} \frac{1}{\omega\left(B\left(0,2^{j} R\right)\right)} \int_{\Omega \cap\{R<|Y|<2 R\}} \frac{u(Y)^{2}}{\delta(Y)} d Y \\
& \leqslant \frac{C}{R}\left(\frac{\delta(X)}{2^{j} R}\right)^{\frac{3}{4}} \frac{1}{\omega\left(B\left(0,2^{j} R\right)\right)} I_{R} \\
& \leqslant C R^{n}\left[\frac{\omega(B(0, R))}{R^{n}}\right]^{2}\left(\frac{\delta(X)}{|X|}\right)^{\frac{3}{4}} \frac{1}{\omega(B(0,|X|))} .
\end{aligned}
$$

Inequality (3.16) is proved by combining (3.40) and (3.41).

Case 3. Let $\frac{1}{2} R<|X|<4 R$. Note that $\delta(X)<4 R$. Let $\widehat{X} \in \partial \Omega$ be such that $\delta(X)=|\widehat{X}-X|$, which implies that $|\widehat{X}|<8 R$. Note that if $Y \in B(0,2 R)$ then $Y \in B(\widehat{X} ; 10 R)$. We now look at

$$
\begin{aligned}
\left|\omega_{R}^{2}(X)\right| \leqslant & \frac{C}{R} \int_{\Omega \cap\{R<|Y|<2 R\} \cap B(\widehat{X}, 10 R)} G(X, Y) \frac{u(Y)}{\delta(Y)^{2}} d Y \\
\leqslant & \frac{C}{R} \int_{\Omega \cap\{R<|Y|<2 R\} \cap B(X, \delta(X) / 2)} G(X, Y) \frac{u(Y)}{\delta^{2}(Y)} d Y \\
& +\frac{C}{R} \int_{\Omega \cap\{R<|Y|<2 R\} \cap(B(\widehat{X}, 2 \delta(X) \backslash B(X, \delta(X) / 2)))} G(X, Y) \frac{u(Y)}{\delta^{2}(Y)} d Y \\
& +\frac{C}{R} \int_{\Omega \cap\{R<|Y|<2 R\} \cap(B(\widehat{X}, 10 R) \backslash B(\widehat{X}, 2 \delta(X)))} G(X, Y) \frac{u(Y)}{\delta^{2}(Y)} d Y .
\end{aligned}
$$

For $Y \in \Omega \cap\{R<|Y|<2 R\} \cap B(X, c \delta(X) / 2)$,

$$
G(X, Y) \leqslant \frac{C}{|X-Y|^{n-1}} \quad \text { and } \quad \frac{u(Y)}{\delta(Y)^{2}} \leqslant C \frac{u(X)}{\delta(X)^{2}},
$$

by Harnack's principle. Thus

$$
\begin{gathered}
\int_{\Omega \cap\{R<|Y|<2 R\} \cap B(X, \delta(X) / 2)} G(X, Y) \frac{u(Y)}{\delta^{2}(Y)} d Y \\
\leqslant C \frac{u(X)}{\delta(X)^{2}} \int_{\Omega \cap\{R<|Y|<2 R\} \cap B(X, \delta(X) / 2)} \frac{d Y}{|X-Y|^{n-1}} \leqslant C u(X) .
\end{gathered}
$$




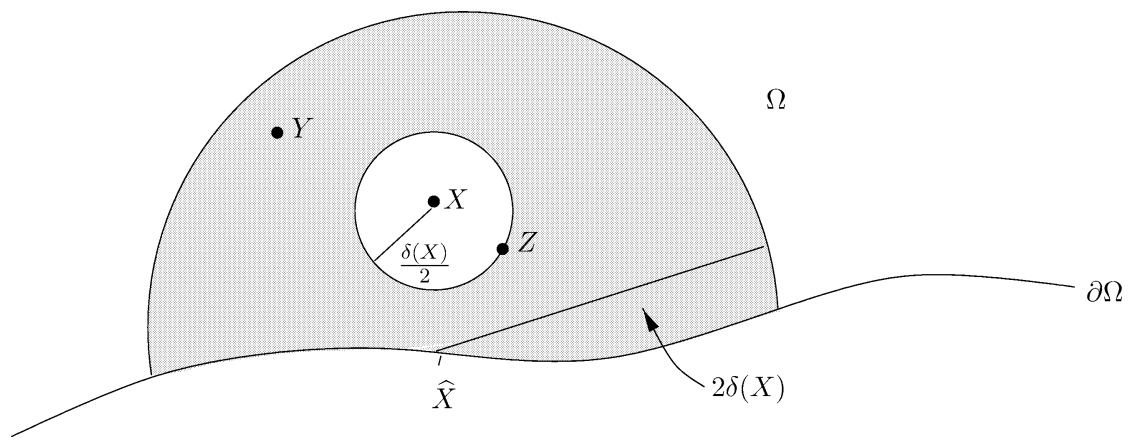

Fig. 1.

If $Y \in \Omega \cap\{R<|Y|<2 R\} \cap[B(\widehat{X}, 2 \delta(X)) \backslash B(X, \delta(X) / 2)]$

$$
G(X, Y) \leqslant C \frac{u(Y)}{u(Z)} G(X, Z) \leqslant C \frac{u(Y)}{u(X)} \frac{1}{\delta(X)^{n-1}}
$$

by the Comparison Principle, for $Z \in \partial B(X, \delta(X) / 2)$ (see Fig. 1). Thus (3.45) yields

$$
\begin{aligned}
& \int_{\Omega \cap\{R<|Y|<2 R\} \cap(B(\widehat{X}, 2 \delta(X)) \backslash B(X, \delta(X) / 2))} G(X, Y) \frac{u(Y)}{\delta(Y)^{2}} d Y \\
& \leqslant C \frac{1}{u(X) \delta(X)^{n-1}} \int_{\Omega \cap B(\widehat{X}, 2 \delta(X))} \frac{u^{2}(Y)}{\delta(Y)^{2}} d Y .
\end{aligned}
$$

A similar argument to the one used to estimate $I_{R}$ (see (3.23)) ensures that

$$
\int_{\Omega \cap B(\widehat{X}, 2 \delta(X))} \frac{u^{2}(Y)}{\delta^{2}(Y)} d Y \leqslant C \frac{u^{2}(X)}{\delta^{2}(X)} \delta^{n+1}(X) .
$$

Thus combining (3.46) and (3.47) we obtain

$$
\int_{\Omega \cap\{R<|Y|<2 R\} \cap(B(\widehat{X}, 2 \delta(X)) \backslash B(X, \delta(X) / 2))} G(X, Y) \frac{u(Y)}{\delta^{2}(Y)} d Y \leqslant C u(X) .
$$

If $Y \in \Omega \cap\{R<|Y|<2 R\} \cap(B(\widehat{X}, 10 R) \backslash B(\widehat{X}, 2 \delta(X)))$ there exists $j \in\left\{1, \ldots, j_{0}\right\}$ so that $2^{j} \delta(X) \leqslant|\widehat{X}-Y|<2^{j+1} \delta(X)$ where $j_{0}$ is such that $2^{j_{0}} \delta(X)>10 R \geqslant 2^{j_{0}-1} \delta(X)$. Let $Y_{j}=A\left(\widehat{X}, 2^{j} \delta(X)\right)$ be a non-tangential point with respect to $\widehat{X}$ at radius $2^{j} \delta(X)$. Then for $Y \in B\left(\widehat{X}, 2^{j+1} \delta(X)\right) \backslash B\left(\widehat{X}, 2^{j} \delta(X)\right)$ by the Comparison Principle, Lemma 2.1 and (3.8) we have

$$
\begin{aligned}
G(X, Y) & \leqslant C G\left(X, Y_{j}\right) \frac{u(Y)}{u\left(Y_{j}\right)} \leqslant C\left(\frac{\delta(X)}{2^{j} \delta(X)}\right)^{\frac{3}{4}} G\left(Y_{j-1}, Y_{j}\right) \frac{u(Y)}{u\left(Y_{j}\right)} \\
& \leqslant C \frac{1}{2^{\frac{3 j}{4}}} \frac{u(Y)}{2^{j(n-1)} \delta(X)^{(n-1)}} \frac{2^{j(n-1)} \delta(X)^{n-1}}{\omega\left(B\left(\widehat{X}, 2^{j} \delta(X)\right)\right)}
\end{aligned}
$$




$$
\leqslant C \frac{u(Y)}{\left(2^{j}\right)^{\frac{3}{4}} \omega\left(B\left(\widehat{X}, 2^{j} \delta(X)\right)\right)} .
$$

Hence using (3.8), Harnack's principle and an argument similar to the one used to prove (3.23) we have that

$$
\begin{aligned}
& \int_{\Omega \cap\{R<|Y|<2 R\} \cap(B(\widehat{X}, 10 R) \backslash B(\widehat{X}, 2 \delta(X)))} G(X, Y) \frac{u(Y)}{\delta^{2}(Y)} d Y \\
& \leqslant C \sum_{j=1}^{j_{0}} \frac{1}{\left(2^{j}\right)^{\frac{3}{4}} \omega\left(B\left(\widehat{X}, 2^{j} \delta(X)\right)\right)} \\
& \times \quad \int \frac{u^{2}(Y)}{\delta^{2}(Y)} d Y \\
& \Omega \cap\{R<|Y|<2 R\} \cap\left\{2^{j} \delta(X) \leqslant|\widehat{X}-Y|<2^{j+1} \delta(X)\right\} \\
& \leqslant C \sum_{j=1}^{j_{0}} \frac{1}{\left(2^{j}\right)^{\frac{3}{4}} \omega\left(B\left(\widehat{X}, 2^{j} \delta(X)\right)\right)} \int_{B\left(\widehat{X}, 2^{j+1} \delta(X)\right) \cap \Omega} \frac{u^{2}(Y)}{\delta^{2}(Y)} d Y \\
& \leqslant C \sum_{j=1}^{j_{0}} \frac{1}{\left(2^{j}\right)^{\frac{3}{4}} \omega\left(B\left(\widehat{X}, 2^{j} \delta(X)\right)\right)}\left(2^{j+1} \delta(X)\right)^{n+1} \frac{u^{2}\left(Y_{j+1}\right)}{\left(2^{j+1} \delta(X)\right)^{2}} \\
& \leqslant C \sum_{j=1}^{j_{0}} \frac{1}{\left(2^{j}\right)^{\frac{3}{4}}} \frac{u\left(Y_{j}\right)^{2}\left(2^{j} \delta(X)\right)^{n-1}}{\omega\left(B\left(\widehat{X}, 2^{j} \delta(X)\right)\right)} \leqslant C \sum_{j=1}^{j_{0}} \frac{1}{\left(2^{j}\right)^{\frac{3}{4}}} \frac{\omega\left(B\left(\widehat{X}, 2^{j} \delta(X)\right)\right)}{\left[2^{j} \delta(X)\right]^{n-1}} .
\end{aligned}
$$

Since $\log h \in \operatorname{VMO}(\partial \Omega)$, by (3.2), and using the fact that $\omega$ is doubling in the case where $j=j_{0}$ we have that

$$
\frac{\omega\left(B\left(\widehat{X}, 2^{j} \delta(X)\right)\right)}{\omega(B(\widehat{X}, 10 R))} \leqslant C\left(\frac{2^{j} \delta(X)}{R}\right)^{n-\frac{1}{8}} .
$$

Thus combining (3.50) and (3.51) we obtain

$$
\begin{gathered}
\int_{\Omega \cap\{R<|Y|<2 R\} \cap(B(\widehat{X}, 10 R) \backslash B(\widehat{X}, 2 \delta(X)))} G(X, Y) \frac{u(Y)}{\delta^{2}(Y)} d Y \\
\leqslant C \omega(B(\widehat{X}, 10 R)) \sum_{j=1}^{j_{0}} \frac{1}{\left(2^{j}\right)^{\frac{3}{4}}} \cdot \frac{1}{R^{n-\frac{1}{8}}} \frac{\left(2^{j} \delta(X)\right)^{n-\frac{1}{8}}}{\left(2^{j} \delta(X)\right)^{n-1}} \\
\leqslant \frac{C}{R^{n-\frac{1}{8}}} \sum_{j=1}^{j_{0}} \delta(X)^{\frac{7}{8}} \frac{\omega(B(\widehat{X}, 10 R))}{\left(2^{j}\right)^{\frac{3}{4}-\frac{7}{8}}} \leqslant C \frac{\delta(X)^{\frac{7}{8}}}{R^{n-\frac{1}{8}}} \omega(B(\widehat{X}, 10 R)) \sum_{j=1}^{j_{0}}\left(2^{j}\right)^{\frac{1}{8}} \\
\leqslant C \frac{\delta(X)^{\frac{7}{8}}}{R^{n-\frac{1}{8}}} \omega(B(\widehat{X}, 10 R))\left(2^{j_{0}}\right)^{\frac{1}{8}} \leqslant C \frac{\delta(X)^{\frac{7}{8}}}{R^{n-\frac{1}{8}}} \omega(B(\widehat{X}, 10 R))\left(\frac{R}{\delta(X)}\right)^{\frac{1}{8}} \\
\leqslant C \frac{\delta(X)^{\frac{3}{4}}}{R^{n}} R^{\frac{1}{4}} \omega(B(\widehat{X}, 10 R)) .
\end{gathered}
$$

Since $\omega$ is a doubling measure and $|\widehat{X}|<8 R$ then

$$
\omega(B(\widehat{X}, 10 R)) \leqslant \omega(B(0,18 R)) \leqslant C \omega(B(0, R)) .
$$


Combining this remark, (3.42), (3.44), (3.48) and (3.52) we obtain that

$$
\left|\omega_{R}^{2}(X)\right| \leqslant \frac{C}{R} u(X)+C\left(\frac{\delta(X)}{R}\right)^{\frac{3}{4}} \frac{\omega(B(0, R))}{R^{n}} .
$$

By the Harnack principle, Corollary 2.2 and (3.8), for $X \in B(0,4 R)$ we have

$$
u(X) \leqslant C\left(\frac{\delta(X)}{R}\right)^{\frac{3}{4}} u\left(A_{R}\right) \leqslant C\left(\frac{\delta(X)}{R}\right)^{\frac{3}{4}} \frac{\omega(B(0, R))}{R^{n-1}} .
$$

Combining (3.53) and (3.54) we obtain

$$
\left|\omega_{R}^{2}(X)\right| \leqslant C\left(\frac{\delta(X)}{R}\right)^{\frac{3}{4}} \frac{\omega(B(0, R))}{R^{n}} .
$$

We now look at $\omega_{R}^{1}(X)$,

$$
\begin{aligned}
\left|\omega_{R}^{1}(X)\right| & \leqslant \frac{C}{R^{2}} \int_{\Omega \cap\{R<|Y|<2 R\} \cap B(\widehat{X}, 10 R)} G(X, Y) \frac{u(Y)}{\delta(Y)} d Y \\
& \leqslant \frac{C}{R} \int_{\Omega \cap\{R<|Y|<2 R\} \cap B(\widehat{X}, 10 R)} G(X, Y) \frac{u(Y)}{\delta(Y)^{2}} d Y .
\end{aligned}
$$

Combining (3.42), (3.44), (3.48), (3.52) and (3.54) we obtain that

$$
\left|\omega_{R}^{1}(X)\right| \leqslant C\left(\frac{\delta(X)}{R}\right)^{\frac{3}{4}} \frac{\omega(B(0, R))}{R^{n}},
$$

which concludes the proof of (3.17), and that of Lemma 3.3. In fact note that (3.15), (3.16) and (3.17) ensure that $\omega_{R}$ vanishes continuously at the boundary, and that $\omega_{R} \in C^{\alpha}(\bar{\Omega})$ for $\alpha \in\left(0, \frac{3}{4}\right)$.

Proof of Theorem 3.1. - Recall that $\Omega$ is an unbounded $\delta$-Reifenberg flat chord arc domain, satisfying Corollaries 2.2 and 2.3 with $\varepsilon=\frac{1}{4}$. Assume that $0 \in \partial \Omega$. Let $R>1$, and using the notation introduced in Lemma 3.3 define for $X \in \Omega$,

$$
h_{R}(X)=\varphi_{R}(X) \nabla u(X)-\omega_{R}(X) .
$$

Note that $h_{R}$ is a harmonic function in $\Omega$ satisfying $h_{R} \equiv 0$ on $\partial \Omega \backslash B(0,2 R)$. In fact (3.14) ensures that

$$
\Delta \omega_{R}=\Delta\left[\varphi_{R} \nabla u\right]
$$

The proof of Lemma 3.1 ensures that $N\left(\varphi_{R}(X) \nabla u(X)\right) \in L^{1}\left(d \omega^{X_{*}}\right)$ for every $X_{*} \in \Omega$. Lemma 3.3 guarantees that $\omega_{R}$ is bounded, thus $N\left(\omega_{R}\right) \in L^{1}\left(d \omega^{X_{*}}\right)$ for every $X_{*} \in \Omega$. Thus $N\left(h_{R}\right) \in L^{1}\left(d \omega^{X_{*}}\right)$ for every $X_{*} \in \Omega$ and Lemma 3.3 in Appendix A ensures that

$$
h_{R}(X)=\int_{\partial \Omega} \varphi_{R}(Q) F(Q) d \omega^{X} \quad \text { for } X \in \Omega .
$$


Therefore for $X \in \Omega \cap B\left(0, \frac{R}{2}\right)$ using (3.15) and Lemma 3.2 we have

$$
|\nabla u(X)| \leqslant\left|h_{R}(X)\right|+\left|\omega_{R}(X)\right| \leqslant \int_{\partial \Omega} h(Q) d \omega^{X}(Q)+C \frac{\delta(X)^{3 / 4}}{R^{1 / 2}} .
$$

Letting $R \rightarrow \infty$ we obtain that for $X \in \Omega$

$$
|\nabla u(X)| \leqslant \int_{\partial \Omega} h(Q) d \omega^{X}(Q)
$$

which proves Theorem 3.1.

Proof of Theorem 3.2. - Let $Q_{0} \in \partial \Omega$. Let $\varphi \in C_{c}^{\infty}\left(B\left(Q_{0}, \delta(A) / 4\right)\right), \varphi \equiv 1$ for

$$
\left|X-Q_{0}\right|<\delta(A) / 8
$$

$0 \leqslant \varphi \leqslant 1,|\nabla \varphi| \leqslant C / \delta(A)$ and $|\Delta \varphi| \leqslant C / \delta(A)^{2}$. In particular $\varphi \equiv 0$ in $B(A, \delta(A) / 4)$. For $X \in \Omega$ define

$$
\omega_{0}(X)=\int_{\Omega} G(X, Y) \Delta[\varphi(Y) \nabla G(A, Y)] d Y .
$$

As in Lemma 3.3 we have that $\left.\omega_{0}\right|_{\partial \Omega} \equiv 0, \omega_{0} \in C^{\alpha}(\bar{\Omega})$, and

$$
\left|\omega_{0}(X)\right| \leqslant \frac{C}{\delta(A)^{n}}\left(\frac{\delta(X)}{\delta(A)}\right)^{\frac{3}{4}} \text { for } X \in \Omega \cap B\left(Q_{0}, \frac{\delta(A)}{4}\right) .
$$

In fact $\omega_{0}(X)=\omega_{0}^{1}(X)+\omega_{0}^{2}(X)$ where

$$
\omega_{0}^{1}(X)=\int_{\Omega} G(X, Y) \Delta \varphi \nabla G(A, Y) d Y
$$

and

$$
\omega_{0}^{2}(X)=\int_{\Omega} G(X, Y) \nabla \varphi \nabla(\nabla G(A, Y)) d Y
$$

Note that

$$
|\nabla G(A, Y)| \leqslant C \frac{G(A, Y)}{\delta(Y)} \text { and }\left|\nabla^{2} G(A, Y)\right| \leqslant C \frac{G(A, Y)}{\delta(Y)^{2}}
$$

also spt $\nabla \varphi$, spt $\Delta \varphi \subset B\left(Q_{0}, 2 R\right) \backslash \overline{B\left(Q_{0}, R\right)}$ where $R=\delta(A) / 8$. For

$$
Y \in \Omega \cap B\left(Q_{0}, 2 R\right) \backslash \overline{B\left(Q_{0}, R\right)}
$$

Corollary 2.3 and the comparison principle ensure that 


$$
\begin{aligned}
G(X, Y) & \leqslant C\left(\frac{\delta(X)}{R}\right)^{\frac{3}{4}} G\left(A_{2 R}, Y\right) \leqslant C G\left(A_{2 R}, A_{R}\right)\left(\frac{\delta(X)}{R}\right)^{\frac{3}{4}} \frac{G(A, Y)}{G\left(A, A_{2 R}\right)} \\
& \leqslant \frac{C}{R^{n-1}}\left(\frac{\delta(X)}{R}\right)^{\frac{3}{4}} \frac{G(A, Y)}{G\left(A, A_{2 R}\right)},
\end{aligned}
$$

where $A_{2 R}=A\left(Q_{0}, R\right)$; i.e. $R / M<\left|A_{2 R}-Q_{0}\right|<2 R$ and $\delta\left(A_{2 R}\right) \geqslant R / M$ and similarly for $A_{R}$. Therefore by Harnack's principle and the fact that $\delta$ is chosen so that $\Omega$ satisfies Corollary 2.3 with $\varepsilon=\frac{1}{4}$ we have

$$
\begin{aligned}
\left|\omega_{0}^{2}(X)\right| & \leqslant \frac{C}{R^{n}}\left(\int_{\Omega \cap\left\{R<\left|Y-Q_{0}\right|<2 R\right\}} \frac{G(A, Y)^{2}}{\delta(Y)^{2}} d Y\right)\left(\frac{\delta(X)}{R}\right)^{\frac{3}{4}} \frac{1}{G\left(A, A_{2 R}\right)} \\
& \leqslant \frac{C}{R^{n}}\left(\frac{\delta(X)}{R}\right)^{\frac{3}{4}} G\left(A, A_{2 R}\right) \frac{1}{R^{\frac{3}{2}}} \int_{\Omega \cap\left\{R<|Y|-Q_{0} \mid<2 R\right\}} \frac{d Y}{\delta(Y)^{\frac{1}{2}}} .
\end{aligned}
$$

The computation done to prove (3.23) shows that

$$
\frac{1}{R^{\frac{3}{2}}} \int_{\Omega \cap\left\{R<\left|Y-Q_{0}\right|<2 R\right\}} \frac{d Y}{\delta(Y)^{\frac{1}{2}}} \leqslant C R^{n-1} .
$$

Combining (3.67), (3.68) and (3.7) we have

$$
\begin{aligned}
\left|\omega_{0}^{2}(X)\right| & \leqslant C\left(\frac{\delta(X)}{R}\right)^{\frac{3}{4}} \frac{G\left(A, A_{2 R}\right)}{R} \leqslant C\left(\frac{\delta(X)}{R}\right)^{\frac{3}{4}} \frac{\omega^{A}\left(B\left(Q_{0}, 2 R\right)\right)}{R^{n}} \\
& \leqslant \frac{C}{R^{n}}\left(\frac{\delta(X)}{R}\right)^{\frac{3}{4}} \omega^{A}\left(B\left(Q_{0}, 4 R\right)\right) .
\end{aligned}
$$

As similar computation shows that the same inequality holds for $\left|\omega_{0}^{1}(X)\right|$, and hence for $X \in \Omega \cap B\left(Q_{0}, \delta(A) / 4\right)$

$$
\left|\omega_{0}(X)\right| \leqslant \frac{C}{R^{n}}\left(\frac{\delta(X)}{R}\right)^{\frac{3}{4}} \omega^{A}\left(B\left(Q_{0}, 4 R\right)\right),
$$

which yields (3.62).

A similar argument as the one presented in the proof of Theorem 3.1 shows that for any $Q_{0} \in \partial \Omega$ and every $X \in \Omega \cap B\left(Q_{0}, \delta(A) / 8\right)$

$$
|\nabla G(A, X)| \leqslant \int_{\partial \Omega} k_{A}(Q) d \omega^{X}(Q)+\frac{C}{\delta(A)^{n}}\left(\frac{\delta(X)}{\delta(A)}\right)^{\frac{3}{4}} \omega^{A}\left(B\left(Q_{0}, \delta(A)\right)\right)
$$

which proves (3.4).

\section{Blow up argument}

In this section, which is the core of the paper, we describe a general construction of blow-up sequences for Reifenberg flat chord arc domains whose Poisson kernels have logarithm in VMO. 
The main result is that any such sequence has a subsequence whose limit satisfies the hypothesis of Theorem 2.2. Let $\Omega \subset \mathbb{R}^{n+1}$ be a $\delta$-Reifenberg flat chord arc domain, with $\delta>0$ small enough so that the conclusion of Corollary 2.3 holds (and that of Corollary 2.2 in the unbounded case) for $\varepsilon=\frac{1}{4}$.

Here again $u$ denotes either the Green function with pole at $A$ or with pole at infinity, $h$ denotes the corresponding Poisson kernel (see (2.27)) and $d \omega=h d \sigma$. We assume that $\log h \in \operatorname{VMO}(\partial \Omega)$. Let $Q_{i} \in \partial \Omega$, and assume $Q_{i} \rightarrow Q_{\infty} \in \partial \Omega$ as $i \rightarrow \infty$. Without loss of generality we may assume that $Q_{\infty}=0$. Let $\left\{r_{i}\right\}_{i \geqslant 1}$ be a sequence of positive numbers so that $\lim _{i \rightarrow \infty} r_{i}=0$. Consider the domains

$$
\Omega_{i}=\frac{1}{r_{i}}\left(\Omega-Q_{i}\right) \quad \text { with } \partial \Omega_{i}=\frac{1}{r_{i}}\left(\partial \Omega-Q_{i}\right)
$$

Consider also the functions $u_{i}$ on $\Omega_{i}$ defined by

$$
u_{i}(Z)=\frac{u\left(r_{i} Z+Q_{i}\right)}{r_{i} \oint_{B\left(Q_{i}, r_{i}\right)} h d \sigma} .
$$

Let $\Omega_{i}^{*}=\Omega_{i}$ if $u$ is the Green's function with pole at infinity and $\Omega^{*}=\Omega_{i} \backslash\left\{\frac{A-Q_{i}}{r_{i}}\right\}$ if $u$ is the Green's function with pole at $A$. Then

$$
\Delta u_{i}=0 \quad \text { on } \Omega_{i}^{*} \subset \Omega_{i},\left.\quad u_{i}\right|_{\partial \Omega_{i}}=0
$$

and

$$
d \omega_{i}(Q)=h_{i}(Q) d \sigma_{i}(Q) \quad \text { for } \mathcal{H}^{n} \text {-a.e. } Q \in \partial \Omega_{i} .
$$

Here $\sigma_{i}=\mathcal{H}^{n} L \partial \Omega_{i}, \omega_{i}$ denotes the harmonic measure of $\Omega_{i}$ either with pole at infinity or at $\frac{A-Q_{i}}{r_{i}}$, depending on whether $u$ is the Green's function with pole at infinity or with pole at $A$. Furthermore the corresponding Poisson kernel $h_{i}$ satisfies

$$
h_{i}(Q)=\frac{h\left(r_{i} Q+Q_{i}\right)}{\oint_{B\left(Q_{i}, r_{i}\right)} h d \sigma} .
$$

Since $\log h \in \mathrm{VMO}$, by including the term $\oint_{B\left(Q_{i}, r_{i}\right)} h d \sigma$ in the denominator of the function $u_{i}$ defined in (4.2) we "remove the singularity" of the Poisson kernel of the limit domain. This is the "correct" type of blow up in the sense that it allows us to connect the geometry of the limit domain to the analytic properties of its Green's function with pole at infinity.

THEOREM 4.1. - There exists a subsequence (which we relabel) satisfying

$$
\Omega_{i} \rightarrow \Omega_{\infty} \text { in the Hausdorff distance sense, uniformly on compact sets, }
$$

where $\Omega_{\infty}$ is an unbounded $4 \delta$-Reifenberg flat chord arc domain. Moreover there exists $u_{\infty} \in C(\bar{\Omega})$ such that

$$
u_{i} \rightarrow u_{\infty} \text { uniformly on compact sets }
$$


and

$$
\begin{cases}\Delta u_{\infty}=0 & \text { in } \Omega_{\infty}, \\ u_{\infty}=0 & \text { in } \partial \Omega_{\infty}, \\ u_{\infty}>0 & \text { in } \Omega_{\infty} .\end{cases}
$$

Furthermore

$$
\omega_{i} \rightarrow \omega_{\infty}
$$

weakly as Radon measures. Moreover $\omega_{\infty}$ is the harmonic measure of $\Omega_{\infty}$ with pole at infinity (corresponding to $u_{\infty}$ ).

Proof. - Since for each $i \geqslant 1, B(0,1) \cap \Omega_{i} \neq \emptyset$ and $0 \in \partial \Omega_{i}$, given a compact set $K \subset \mathbb{R}^{n+1}$, there exists a subsequence $\left\{i^{\prime}\right\}$ such that $\Omega_{i^{\prime}} \cap K$ and $\partial \Omega_{i}^{\prime} \cap K$ converge in the Hausdorff distance sense. Taking an exhaustion of $\mathbb{R}^{n+1}$ by compact sets, we can insure that there exists another subsequence $\left\{i_{k}\right\}$ such that $\Omega_{i_{k}}$ and $\partial \Omega_{i_{k}}$ converge in the Hausdorff distance sense, uniformly on compact sets. Hence modulo relabeling the subsequence we have that

$$
\Omega_{i} \rightarrow \Omega_{\infty} \text { in the Hausdorff distance sense uniformly on compact sets, }
$$

and

$$
\partial \Omega_{i} \rightarrow \Sigma_{\infty} \text { in the Hausdorff distance sense uniformly on compact sets. }
$$

Note that if $E \subset \mathbb{R}^{n+1}$ is a Borel set

$$
\begin{aligned}
\omega_{i}(E) & =\int_{E} h_{i}(Q) d \sigma_{i}(Q)=\frac{\int_{E} h\left(r_{i} Q+Q_{i}\right) d \sigma_{i}(Q)}{\oint_{B\left(Q_{i}, r_{i}\right)} h d \sigma} \\
& =\frac{r_{i}^{-n} \int_{r_{i} E+Q_{i}} h(Q) d \sigma(Q)}{\oint_{B\left(Q_{i}, r_{i}\right)} h(Q) d \sigma(Q)}=r_{i}^{-n} \sigma\left(B\left(Q_{i}, r_{i}\right)\right) \frac{\omega\left(r_{i} E+Q_{i}\right)}{\omega\left(B\left(Q_{i}, r_{i}\right)\right)} .
\end{aligned}
$$

Since $\partial \Omega$ is Ahlfors regular, there exists $C>1$ so that

$$
C^{-1} \frac{\omega\left(r_{i} E+Q_{i}\right)}{\omega\left(B\left(Q_{i}, r_{i}\right)\right)} \leqslant \omega_{i}(E) \leqslant C \frac{\omega\left(r_{i} E+Q_{i}\right)}{\omega\left(B\left(Q_{i}, r_{i}\right)\right)} .
$$

Since $\omega$ is a doubling measure for each compact set $K \subset \mathbb{R}^{n+1}, \sup _{i \geqslant 1} \omega_{i}(K) \leqslant C_{K}$. Hence there exists a subsequence (which we relabel again) so that $\omega_{i} \rightarrow \omega_{\infty}$ and $\mu_{i} \rightarrow \mu_{\infty}$ where $\mu_{i}(E)=\frac{\omega\left(r_{i} E+Q_{i}\right)}{\omega\left(B\left(Q_{i}, r_{i}\right)\right)}$. Note that $C^{-1} \mu_{\infty} \leqslant \omega_{\infty} \leqslant C \mu_{\infty}$ which ensures that $\operatorname{spt} \mu_{\infty}=\operatorname{spt} \omega_{\infty}$, where spt denotes the support of a measure. Our immediate goal is to show that $\Sigma_{\infty}=\partial \Omega_{\infty}$, to do this we first need to prove that $\Sigma_{\infty}=\operatorname{spt} \omega_{\infty}$. It is straightforward to show that spt $\mu_{\infty} \subset \Sigma_{\infty}$ (see proof of Lemma 2.1 in [18]). Now assume that $X \in \Sigma_{\infty}$, there exist $X_{i}=\frac{1}{r_{i}}\left(Z_{i}-Q_{i}\right) \in \partial \Omega_{i}$ with $Z_{i} \in \partial \Omega$ so that $X_{i} \rightarrow X$. For $r \in(0,1)$ there exists $i_{0} \geqslant 1$ so that for $i \geqslant i_{0}\left|X-X_{i}\right|<\frac{r}{2}$ and $\left|Z_{i}-Q_{i}\right| \leqslant M r_{i}$, where $M=|X|+1$. Then for $i \geqslant i_{0}$

$$
\begin{aligned}
\mu_{i}(B(X, r)) & =\frac{\omega\left(B\left(r_{i} X+Q_{i} ; r r_{i}\right)\right)}{\omega\left(B\left(Q_{i}, r_{i}\right)\right)} \geqslant \frac{\omega\left(B\left(Z_{i}, \frac{r}{2} r_{i}\right)\right)}{\omega\left(B\left(Q_{i}, r_{i}\right)\right)} \\
& \geqslant \frac{\omega\left(B\left(Z_{i}, \frac{r}{2} r_{i}\right)\right)}{\omega\left(B\left(Z_{i}, r_{i}(M+1)\right)\right)} \geqslant C(r, M),
\end{aligned}
$$

$4^{\mathrm{e}}$ SÉRIE - TOME $36-2003-\mathrm{N}^{\circ} 3$ 
because $\omega$ is doubling. From (4.15) we deduce that $X \in \operatorname{spt} \mu_{\infty}$, which combined with the remarks above ensures that $\Sigma_{\infty}=\operatorname{spt} \omega_{\infty}$. In order to prove that $\partial \Omega_{\infty}=\Sigma_{\infty}$, let

$$
X \in \partial \Omega_{\infty}=\overline{\Omega_{\infty}} \cap \overline{\Omega_{\infty}^{c}} .
$$

Given $\varepsilon>0$ there exist $Y \in \Omega_{\infty} \cap B(X, \varepsilon)$ and $Y^{\prime} \in \Omega_{\infty}^{c} \cap B(X, \varepsilon)$. By definition

$$
Y^{\prime}=\lim _{i \rightarrow \infty} \frac{1}{r_{i}}\left(Y_{i}^{\prime}-Q_{i}\right)
$$

for some $Y_{i}^{\prime} \in \Omega^{c}$. Moreover there exists a sequence $Y_{i} \in \mathbb{R}^{n+1}$ such that

$$
Y=\lim _{i \rightarrow \infty} \frac{1}{r_{i}}\left(Y_{i}-Q_{i}\right)
$$

Modulo taking a subsequence we may assume that $Y_{i} \in \Omega$. A simple connectivity argument shows that for each $i \geqslant 1$ there exists $P_{i} \in \partial \Omega \cap\left[Y_{i}, Y_{i}^{\prime}\right]$, where $\left[Y_{i}, Y_{i}^{\prime}\right]$ denotes the segment joining $Y_{i}$ to $Y_{i}^{\prime}$. Let $P_{i}=\left(1-t_{i}\right) \frac{1}{r_{i}} Y_{i}+t_{i} \frac{1}{r_{i}} Y_{i}^{\prime}$ for some $t_{i} \in(0,1)$ then the sequence

$$
\frac{1}{r_{i}}\left(P_{i}-Q_{i}\right)=\left(1-t_{i}\right) \frac{1}{r_{i}}\left(Y_{i}-Q_{i}\right)+t_{i} \frac{1}{r_{i}}\left(Y_{i}^{\prime}-Q_{i}\right)
$$

is bounded, thus there exists a subsequence $\left\{i_{\varepsilon}\right\}$ such that $\frac{1}{r_{i_{\varepsilon}}}\left(P_{i_{\varepsilon}}-Q_{i_{\varepsilon}}\right) \rightarrow Z_{\varepsilon} \in \Sigma_{\infty}$. Moreover since

$$
\left|\frac{1}{r_{i_{\varepsilon}}}\left(P_{i_{\varepsilon}}-Q_{i_{\varepsilon}}\right)-\frac{1}{r_{i_{\varepsilon}}}\left(Y_{i_{\varepsilon}}-Q_{i_{\varepsilon}}\right)\right| \leqslant \frac{\left|Y_{i_{\varepsilon}}-Y_{i_{\varepsilon}}^{\prime}\right|}{r_{i_{\varepsilon}}},
$$

letting $i_{\varepsilon} \rightarrow \infty$ we have that

$$
\left|Y-Z_{\varepsilon}\right| \leqslant\left|Y-Y^{\prime}\right| \text { and }\left|X-Z_{\varepsilon}\right| \leqslant|X-Y|+\left|Y-Y^{\prime}\right| \leqslant 3 \varepsilon .
$$

Summarizing we have proved that given $X \in \partial \Omega_{\infty}$ and given $\varepsilon>0$ there exists $Z \in \Sigma_{\infty}$ such that $|X-Z|<\varepsilon$. Hence $X \in \bar{\Sigma}_{\infty}=\overline{\operatorname{spt} \omega_{\infty}}=\operatorname{spt} \omega_{\infty}=\Sigma_{\infty}$, i.e., $\partial \Omega_{\infty} \subset \Sigma_{\infty}$. In order to prove the other inclusion we use the fact that since $\Omega$ is a $\delta$-Reifenberg flat domain then $\Omega$ is an NTA domain.

Let $X \in \Sigma_{\infty}$ there exists a sequence $X_{i} \in \partial \Omega$ such that $\frac{1}{r_{i}}\left(X_{i}-Q_{i}\right) \rightarrow X$. Given $\rho>0$ since both $\Omega$ and $\Omega^{c}$ satisfy the corkscrew condition for $i$ large enough (so that $r_{i} \rho<R$ ) there exist $A_{i} \in \Omega$ and $A_{i}^{\prime} \in \Omega^{c}$ such that

$$
\begin{aligned}
& B\left(A_{i}, \frac{r_{i} \rho}{M}\right) \subset \Omega, \quad \text { and } \quad\left|A_{i}-X_{i}\right| \leqslant \rho r_{i}, \\
& B\left(A_{i}^{\prime}, \frac{r_{i} \rho}{M}\right) \subset \Omega^{c}, \quad \text { and } \quad\left|A_{i}^{\prime}-X_{i}\right| \leqslant \rho r_{i},
\end{aligned}
$$

which implies that

$$
\begin{gathered}
B\left(\frac{A_{i}-Q_{i}}{r_{i}}, \frac{\rho}{M}\right) \subset \Omega_{i}, \quad\left|\frac{A_{i}-Q_{i}}{r_{i}}-\frac{X_{i}-Q_{i}}{r_{i}}\right| \leqslant \rho, \\
\operatorname{dist}\left(\frac{A_{i}-Q_{i}}{r_{i}}, \Omega_{i}\right) \geqslant \frac{\rho}{M}
\end{gathered}
$$




$$
\begin{gathered}
B\left(\frac{A_{i}^{\prime}-Q_{i}}{r_{i}}, \frac{\rho}{M}\right) \subset \Omega_{i}^{c}, \quad\left|\frac{A_{i}^{\prime}-Q_{i}}{r_{i}}-\frac{X_{i}-Q_{i}}{r_{i}}\right| \leqslant \rho, \\
\operatorname{dist}\left(\frac{A_{i}^{\prime}-Q_{i}}{r_{i}}, \Omega_{i}\right) \geqslant \frac{\rho}{M} .
\end{gathered}
$$

Modulo passing to a subsequence we may assume that

$$
\frac{A_{i}-Q_{i}}{r_{i}} \rightarrow A_{\infty}(\rho) \in \Omega_{\infty}, \quad \text { and } \quad \frac{A_{i}^{\prime}-Q_{i}}{r_{i}} \rightarrow A_{\infty}^{\prime}(\rho) .
$$

Let $i \rightarrow \infty$ in (4.16) and (4.17) we obtain

$$
B\left(A_{\infty}(\rho), \frac{\rho}{M}\right) \subset \Omega_{\infty}, \quad\left|A_{\infty}(\rho)-X\right| \leqslant \rho,
$$

and

$$
\left|A_{\infty}^{\prime}(\rho)-X\right| \leqslant \rho, \quad \operatorname{dist}\left(A_{\infty}^{\prime}(\rho), \Omega_{\infty}\right) \geqslant \frac{\rho}{2 M} .
$$

(4.18) and (4.19) prove that there exists $M^{\prime}>1$ such that given $X \in \Sigma_{\infty}$ and $\rho>0$ there exist $A_{\infty}(\rho) \in \Omega_{\infty}$ and $A_{\infty}^{\prime}(\rho) \in \Omega_{\infty}^{c}$ such that

$$
\left|A_{\infty}(\rho)-X\right| \leqslant \rho, \quad\left|A_{\infty}^{\prime}(\rho)-X\right| \leqslant \rho,
$$

and

$$
B\left(A_{\infty}(\rho), \frac{\rho}{M}\right) \subset \Omega_{\infty}, \quad B\left(A_{\infty}^{\prime}(\rho), \frac{\rho}{M}\right) \subset \Omega_{\infty}^{c} .
$$

Letting $\rho$ tend to 0 , and using (4.21) we conclude that $X \in \partial \Omega_{\infty}$ hence $\partial \Omega_{\infty}=\Sigma_{\infty}$.

The fact that $\partial \Omega_{\infty}$ is a $4 \delta$-Reifenberg flat set is a direct consequence of the fact that $\partial \Omega$ is a $\delta$-Reifenberg flat set and that the quantity $\theta(Q, r)$ is scale invariant. Let $K \subset \mathbb{R}^{n+1}$ be a compact set, since $\partial \Omega$ is a $\delta$-Reifenberg flat set there exists $R_{K}$ so that for every

$$
Q \in\left\{X \in \mathbb{R}^{n+1}, \operatorname{dist}(X, K) \leqslant 1\right\} \cap \partial \Omega
$$

and $r \in\left(0, R_{K}\right), \theta(Q, r) \leqslant \delta$, i.e., given $\varepsilon>0$ there exists an $n$-plane $L$ containing $Q$ so that

$$
\frac{1}{r} D[\partial \Omega \cap B(Q, r) ; L(Q, r) \cap B(Q, r)]<\delta+\varepsilon .
$$

Let $P \in K \cap \partial \Omega_{\infty}$, there exists a sequence $\left\{P_{i}\right\} \in \partial \Omega$ so that $\lim _{i \rightarrow \infty} \frac{1}{r_{i}}\left(P_{i}-Q_{i}\right)=P$, note that since by assumption $\lim _{i \rightarrow \infty} Q_{i}=0$ then $\lim _{i \rightarrow \infty} P_{i}=0$.

Let $r \in\left(0, R_{K}\right)$ be fixed. Since $\partial \Omega_{i} \rightarrow \partial \Omega_{\infty}$ in the Hausdorff distance sense there exists $r_{0} \geqslant 1$ so that for $i \geqslant i_{0}$ and $r^{\prime} \in((1-\varepsilon) r, r)$

$$
D\left[\partial \Omega_{i} \cap \overline{B\left(P, r^{\prime}\right)} ; \partial \Omega_{\infty} \cap \overline{B\left(P, r^{\prime}\right)}\right]<\varepsilon r,
$$

and if $X_{i}=\frac{1}{r_{i}}\left(P_{i}-Q_{i}\right),\left|X_{i}-P\right|<\varepsilon r$. For $i \geqslant i_{0}$ let $\Lambda_{i}=L\left(P_{i}, r_{i} r\right)-P_{i}+P$ then

$$
\begin{aligned}
D\left[\partial \Omega_{\infty} \cap B(P, r) ; \Lambda_{i} \cap B(P, r)\right] \leqslant & D\left[\partial \Omega_{\infty} \cap B(P, r) ; \partial \Omega_{i} \cap B(P, r)\right] \\
& +D\left[\partial \Omega_{i} \cap B(P, r), \Lambda_{i} \cap B(P, r)\right] .
\end{aligned}
$$


Note that (4.23) implies that

$$
D\left[\partial \Omega_{\infty} \cap B(P, r) ; \partial \Omega_{i} \cap B(P, r)\right] \leqslant \varepsilon r .
$$

Moreover by our choice of $\Lambda_{i}$ since

$$
\begin{aligned}
\frac{1}{r} D & {\left[\partial \Omega_{i} \cap B\left(X_{i}, r\right) ; \Lambda_{i}-P+X_{i} \cap B\left(X_{i}, r\right)\right] } \\
& =\frac{1}{r_{i} r} D\left[\partial \Omega \cap B\left(P_{i} r_{i} r\right) ; L\left(P_{i}, r_{i} r\right) \cap B\left(P_{i}, r_{i} r\right)\right]
\end{aligned}
$$

we have, as in the proof of Theorem 2.2 in [18], that

$$
\begin{aligned}
\partial \Omega_{i} \cap B(P, r) & \subset \partial \Omega_{i} \cap B\left(X_{i}, r(1+\varepsilon)\right) \\
& \subset\left(\Lambda_{i} \cap B\left(X_{i}, r(1+\varepsilon)\right) ; 2 \delta r(1+\varepsilon)+2 \varepsilon r\right) \\
& \subset\left(\Lambda_{i} \cap B(P, r) ; 2 \delta r(1+\varepsilon)+5 \varepsilon_{r}\right),
\end{aligned}
$$

and

$$
\Lambda_{i} \cap B(P, r) \subset\left(\partial \Omega_{i} \cap B(P, r) ; 2 \delta r+4 \varepsilon r\right) .
$$

Hence combining (4.26) and (4.27) we have

$$
D\left[\partial \Omega_{i} \cap B(P, r) ; \Lambda_{i} \cap B(P, r)\right] \leqslant 4 \delta r+10 \varepsilon r .
$$

Combining (4.24), (4.25) and (4.28) we obtain

$$
\frac{1}{r} D\left[\partial \Omega_{\infty} \cap B(P, r) ; \Lambda_{i} \cap B(P, r)\right] \leqslant 4 \delta+11 \varepsilon .
$$

Thus

$$
\theta_{\partial \Omega_{\infty}}(P, r) \leqslant 4 \delta .
$$

The fact that $\partial \Omega_{\infty}$ is a $4 \delta$-Reifenberg set combined with (4.21) ensures that $\Omega_{\infty}$ satisfies the separation property and therefore $\Omega_{\infty}$ is a $4 \delta$-Reifenberg flat domain. Since $\partial \Omega$ is Ahlfors regular, and the measure theoretic boundary of $\Omega$ coincides with its topological boundary, we have that for each $R>0$

$$
\sup _{i \geqslant 1} \sigma_{i}(B(0, R))=\sup _{i \geqslant 1} \frac{\sigma\left(B\left(0, R r_{i}\right)\right)}{r_{i}^{n}} \leqslant C .
$$

The compactness theorem for BV functions (see [7, §5.2.3]), guarantees that (modulo passing to a subsequence) $\chi_{\Omega_{j}} \rightarrow \chi_{E}$ in $L_{\text {loc }}^{1}\left(\mathbb{R}^{n+1}\right)$ where $E$ is a set of locally finite perimeter. We claim that $E=\Omega_{\infty}$. First note that since $\partial \Omega_{\infty}$ has $\mathcal{H}^{n+1}$ measure zero, we may assume that $E \cap \partial \Omega_{\infty}=\emptyset$. We can also assume that all points of $E$ are density points for $\chi_{E}$. Let $X \in \operatorname{int} \Omega_{\infty}^{c}$, there exists $r>0$ so that $B(X, r) \subset \Omega_{\infty}^{c}$. Since $\bar{\Omega}_{i} \rightarrow \bar{\Omega}_{\infty}$ in the Hausdorff distance sense there is $i_{0} \geqslant 1$ so that for $i \geqslant i_{0}, B\left(X, \frac{r}{2}\right) \cap \bar{\Omega}_{i}=\emptyset$, therefore $\mathcal{H}^{n+1}\left(B\left(X, \frac{r}{2}\right) \cap E\right)=0$ thus $X \notin E$. Hence $E \subset \Omega_{\infty}$. Let $X \in \operatorname{int} \Omega_{\infty}$ there exists $r>0, B(X, r) \subset \Omega_{\infty}$, since $\partial \Omega_{i} \rightarrow \partial \Omega_{\infty}$ in the Hausdorff distance there exists $i_{0} \geqslant 1$ so that for $i \geqslant i_{0} ; B\left(X, \frac{r}{2}\right) \cap \partial \Omega_{i}=\emptyset$. Let $P_{i} \in \partial \Omega_{i}$ so that $\rho_{i}=\left|X-P_{i}\right|=\operatorname{dist}\left(X, \partial \Omega_{i}\right) \geqslant \frac{r}{2}$. Since $\Omega_{i}$ satisfies the separation property then either $B\left(X, \frac{r}{4}\right) \subset \Omega_{i}$ or $B\left(X, \frac{r}{4}\right) \subset \Omega_{i}^{c}$. Since $X \in \Omega_{\infty}$, we conclude that for $i$ large $B\left(X, \frac{r}{4}\right) \subset \Omega_{i}$ and 
therefore for $\rho<\frac{r}{4}$,

$$
\mathcal{H}^{n+1}\left(B(X, \rho) \cap \Omega_{i}\right)=\omega_{n+1} \rho^{n+1} \rightarrow \mathcal{H}^{n+1}(B(X, \rho) \cap E) .
$$

Thus $X$ is a density point for $\chi_{E}$, which implies that $X \in E$. We have shown that $\chi_{\Omega_{i}} \rightarrow \chi_{\Omega_{\infty}}$ in $L_{\text {loc }}^{1}\left(\mathbb{R}^{n+1}\right)$ and that $\Omega_{\infty}$ is a set of locally finite perimeter.

Once again since $\Omega_{\infty}$ is a $4 \delta$-Reifenberg flat domain its measure theoretic boundary coincides with its topological boundary (se Remark 4.2 in [18]). This fact combined with the lower semicontinuity of the variation measure (see [7, §5.2.1]) ensures that for $X \in \partial \Omega_{\infty}$ and $r>0$

$$
\sigma_{\infty}(B(X, r)) \leqslant \liminf _{i \rightarrow \infty} \sigma_{i}(B(X, r)) \leqslant \liminf _{i \rightarrow \infty} \frac{\sigma\left(B\left(r_{i} X+Q_{i} ; r r_{i}\right)\right)}{r_{i}^{n}}
$$

where $\sigma_{\infty}=\mathcal{H}^{n}\left\llcorner\partial \Omega_{\infty}\right.$. Since $X \in \partial \Omega_{\infty}$ there exists $X_{i} \in \partial \Omega_{i}$ so that $\lim _{i \rightarrow \infty} X_{i}=X$ and $r_{i} X_{i}+Q_{i}=P_{i} \in \partial \Omega$. Thus since $\partial \Omega$ is Ahlfors regular

$$
\frac{\sigma\left(B\left(r_{i} X+Q_{i}, r r_{i}\right)\right)}{r_{i}^{n}} \leqslant \frac{\sigma\left(B\left(P_{i}, r r_{i}+r_{i}\left|X-X_{i}\right|\right)\right)}{r_{i}^{n}} \leqslant C\left(r+\left|X-X_{i}\right|\right)^{n} .
$$

Combining (4.32) and (4.33) we have that

$$
\sigma_{\infty}(B(X, r)) \leqslant C r^{n}
$$

Since $\Omega_{\infty}$ is an unbounded $4 \delta$-Reifenberg flat domain Remark 4.1 in [18] ensures that for $X \in \partial \Omega_{\infty}$ and $r>0$

$$
\sigma_{\infty}(B(X, r)) \geqslant(1+4 \delta)^{-1} \omega_{n} r^{n}
$$

Therefore $\Omega_{\infty}$ is an unbounded $4 \delta$-Reifenberg flat chord arc domain.

We now prove (4.8), (4.9) and (4.10). The uniqueness of the harmonic measure with finite pole as well as the fact that the composition of a translation and a dilation with a harmonic function is still a harmonic function allows to prove that for $\varphi \in C_{c}^{\infty}\left(\mathbb{R}^{n+1}\right)$ so that $A_{i}=\frac{A-Q_{i}}{r_{i}} \notin \operatorname{spt} \varphi$.

$$
\int_{\partial \Omega} \varphi\left(\frac{P-Q_{i}}{r_{i}}\right) d \omega^{A}(P)=\int_{\Omega_{i}} \varphi(P) d \omega^{A}\left(r_{i} P+Q_{i}\right)=\int_{\Omega_{i}} \Delta \varphi(Z) G_{i}\left(A_{i}, Z\right) d Z
$$

Here $G_{i}\left(A_{i},-\right)$ denotes the Green's function of $\Omega_{i}$ with pole at $A_{i}$. Combining (4.36) and (4.13) we obtain

$$
\int_{\partial \Omega_{i}} \varphi(P) \frac{d \omega^{A}\left(r_{i} P+Q_{i}\right)}{\omega^{A}\left(B\left(Q_{i}, r_{i}\right)\right)}=\int_{\Omega_{i}} \Delta \varphi(Z) \frac{G_{i}\left(A_{i}, Z\right)}{\omega_{i}^{A_{i}}(B(0,1))} d Z .
$$

From (4.4), (4.13) and (4.37) we deduce

$$
\int_{\partial \Omega_{i}} \varphi(P) d \omega_{i}(P)=\frac{\sigma\left(B\left(Q_{i}, r_{i}\right)\right)}{r_{i}^{n}} \int_{\Omega_{i}} \Delta \varphi(Z) \frac{G_{i}\left(A_{i}, Z\right)}{\omega_{i}^{A_{i}}(B(0,1))} d Z .
$$

In particular

$$
h_{i}=\frac{\sigma\left(B\left(Q_{i}, r_{i}\right)\right)}{r_{i}^{n}} \frac{k_{i}^{A_{i}}}{\omega_{i}^{A_{i}}(B(0,1))}
$$


where $k_{i}^{A_{i}}$ denotes the Poisson kernel of $\Omega_{i}$ with pole at $A_{i}$. Since $\Omega_{i}$ is an NTA domain Lemma 4.8 in [14] guarantees that for $i$ large enough (so $A_{i}$ is far enough from $B(0,1)$ ) we have

$$
G_{i}\left(A_{i}, A_{i}(0,1)\right) \sim \omega_{i}^{A_{i}}(B(0,1)),
$$

where $A_{i}(0,1)$ denotes a non-tangential point for $\Omega_{i}$ at 0 and radius 1 .

Since $\partial \Omega$ is Ahlfors regular, the Harnack principle combined with (4.40) asserts the sequence $\left\{\phi_{i}\right\}_{i \geqslant 1}$ of non-negative harmonic functions

$$
\phi_{i}(Z)=\frac{\sigma\left(B\left(Q_{i}, r_{i}\right)\right)}{r_{i}^{n}} \frac{G_{i}\left(A_{i}, Z\right)}{\omega_{i}^{A_{i}}(B(0,1))}
$$

defined for $Z \in B\left(0 ;\left|A_{i}\right|\right) \cap \Omega_{i}$ is uniformly bounded on compact sets. In fact if we let $\phi_{i} \equiv 0$ in $\Omega_{i}^{c}$, by our choice of $\delta>0$, Corollary 2.3 ensures that $\left\{\phi_{i}\right\}$ is uniformly bounded on compact sets in the $C^{3 / 4}$ norm. Moreover $\phi_{i}\left(\frac{A\left(Q_{i}, r_{i}\right)-Q_{i}}{r_{i}}\right) \geqslant C^{-1}$. By the Arzela-Ascoli theorem there exists a subsequence such that $\phi_{i}$ converges to a limit $u_{\infty}$ uniformly on compact sets. Moreover $\Delta u_{\infty}=0$ in $\Omega_{\infty}$ because $\Omega_{i} \rightarrow \Omega_{\infty}$. Since $\phi_{i} \equiv 0$ on $\Omega_{i}^{c}$ and $\Omega_{i}^{c} \rightarrow \Omega_{\infty}^{c}$ in the Hausdorff distance sense, $u_{\infty}=0$ on $\partial \Omega_{\infty}$. Thus $u_{\infty}$ satisfies (4.9). Letting $i \rightarrow \infty$ in (4.38) we conclude that for $\varphi \in C_{c}^{\infty}\left(\mathbb{R}^{n+1}\right)$

$$
\int_{\partial \Omega_{\infty}} \varphi d \omega_{\infty}=\int_{\Omega_{\infty}} u_{\infty}(X) \Delta \varphi(X) .
$$

Now note that if $u(X)=G(A, X)$ then by the uniqueness of the Green's function with finite pole, for $n \geqslant 2$

$$
G\left(A, r_{i} Z+Q_{i}\right)=\frac{1}{r_{i}^{n-1}} G_{i}\left(A_{i}, Z\right) .
$$

Therefore if $u$ denotes the Green's function with pole at $A$, we have that $u_{i}=\phi_{i}$ which proves (4.8), (4.9) and (4.10) in this case.

If $\Omega$ is an unbounded $\delta$-Reifenberg flat chord arc domain and $u$ denotes the Green's function of $\Omega$ with pole at infinity, Lemma 4.8 in [14] combined with the construction described in the proof of Lemma 3.7 in [18] we have that

$$
C^{-1}<\frac{\omega\left(B\left(Q_{i}, r_{i}\right)\right)}{r_{i}^{n-1} u\left(A\left(Q_{i}, r_{i}\right)\right)}<C,
$$

where $A\left(Q_{i}, r_{i}\right)$ denotes a non-tangential point for $\partial \Omega$ at $Q_{i}$ and radius $r_{i}$. The boundary Harnack principle for NTA domains implies that for $X \in B(0, R) \cap \Omega_{i}$

$$
u\left(r_{i} X+Q_{i}\right) \leqslant C_{R} u\left(A\left(Q_{i}, r_{i}\right)\right) .
$$

Thus combining (4.44), (4.45) and the fact that $\partial \Omega$ is Ahlfors regular we obtain that for $X \in B(0, R) \cap \Omega_{i}$

$$
\begin{aligned}
u_{i}(X) & =\frac{u\left(r_{i} X+Q_{i}\right)}{r_{i} \oint_{B\left(Q_{i}, r_{i}\right)} h d \sigma} \leqslant C_{R} \sigma\left(B\left(Q_{i}, r_{i}\right)\right) \frac{u\left(A\left(Q_{i}, r_{i}\right)\right)}{r_{i} \omega\left(B\left(Q_{i}, r_{i}\right)\right)} \\
& \leqslant C_{R} \frac{r_{i}^{n-1}}{\omega\left(B\left(Q_{i}, r_{i}\right)\right)} u\left(A\left(Q_{i}, r_{i}\right)\right) \leqslant C_{R}
\end{aligned}
$$


Thus $\left\{u_{i}\right\}$ is uniformly bounded on compact sets, $u_{i}\left(\frac{A\left(Q_{i}, r_{i}\right)-Q_{i}}{r_{i}}\right) \geqslant C^{-1}$, by the Arzela-Ascoli theorem $u_{i} \rightarrow u_{\infty}$ uniformly on compact sets and satisfies (4.9). To show that $\omega_{\infty}$ is the harmonic measure with pole at $\infty$ associated to $u_{\infty}$, note that for $\varphi \in C_{c}^{\infty}\left(\mathbb{R}^{n+1}\right)$

$$
\int_{\Omega_{i}} \Delta \varphi(X) u\left(r_{i} X+Q_{i}\right) d X=\frac{1}{r_{i}^{n-1}} \int_{\partial \Omega_{i}} \varphi(X) d \omega\left(r_{i} X+Q_{i}\right),
$$

hence

$$
\begin{aligned}
\int_{\Omega_{i}} \Delta \varphi(X) u_{i}(X) d x & =\frac{\sigma\left(B\left(Q_{i}, r_{i}\right)\right)}{r_{i}^{n}} \int_{\partial \Omega_{i}} \varphi(X) \frac{d \omega\left(r_{i} X+Q_{i}\right)}{\omega\left(B\left(Q_{i}, r_{i}\right)\right)} \\
& =\int_{\partial \Omega_{i}} \varphi(X) d \omega_{i}(X) .
\end{aligned}
$$

Letting $i \rightarrow \infty$, using the fact that $u_{i} \rightarrow u_{\infty}, \omega_{i} \rightarrow \omega_{\infty}, \Omega_{i} \rightarrow \Omega_{\infty}$ and $\partial \Omega_{i} \rightarrow \partial \Omega_{\infty}$ we conclude that

$$
\int_{\Omega_{\infty}} u_{\infty}(X) \Delta \varphi(X) d X=\int_{\partial \Omega_{\infty}} \varphi d \omega_{\infty}
$$

THEOREM 4.2. - If $\Omega_{\infty} \subset \mathbb{R}^{n+1}$ and $u_{\infty}$ are as in Theorem 4.1, then

$$
\sup _{Z \in \Omega_{\infty}}\left|\nabla u_{\infty}(Z)\right| \leqslant 1
$$

The proof of Theorem 4.2 will be done by establishing a series of lemmas. Using the notation above we have:

Lemma 4.1. - Given $\varepsilon>0, A>1$ we have

$$
\sup _{i \geqslant 1} \int_{\partial \Omega_{i} \cap\{|Q|<A\}} h_{i}(Q) d \sigma_{i}(Q) \leqslant C A^{n(1+\varepsilon)},
$$

where $C$ is a constant that depends on $\varepsilon$ and $n$.

Proof. - This is a straightforward consequence of Corollary 2.4. Note that (4.13) combined with (2.23) yields

$$
\int_{\partial \Omega_{i} \cap\{|Q|<A\}} h_{i}(Q) d \sigma_{i}(Q)=\frac{\sigma\left(B\left(Q_{i}, r_{i}\right)\right)}{r_{i}^{n}} \cdot \frac{\omega\left(B\left(Q_{i}, A r_{i}\right)\right)}{\omega\left(B\left(Q_{i}, r_{i}\right)\right)}
$$

because $\partial \Omega$ is Ahlfors regular.

$$
\leqslant C_{\varepsilon}\left(\frac{\sigma\left(B\left(Q_{i}, A r_{i}\right)\right)}{\sigma\left(B\left(Q_{i}, r_{i}\right)\right)}\right)^{1+\varepsilon} \leqslant C_{\varepsilon} A^{n(1+\varepsilon)}
$$

LEMMA 4.2. - Let $Z \in \Omega_{\infty}$. Given $\varepsilon>0$ there exists $i_{0} \geqslant 1$ so that

$$
\eta=\inf _{i \geqslant i_{0}} d\left(Z, \partial \Omega_{i}\right)>0
$$

and there exists $M=M(|Z|, \eta, \varepsilon)>0$ such that 


$$
\sup _{i \geqslant i_{0}} \int_{\partial \Omega_{i} \cap\{|Q|>M\}} h_{i}(Q) d \omega_{i}^{Z}(Q)<\varepsilon .
$$

Here $\omega_{i}^{Z}$ denotes the harmonic measure of $\Omega_{i}$ with pole at $Z$.

Proof. - Let us first remark that if $Z \in \Omega_{\infty}$ then $Z \in \Omega_{i}$ for $i$ large enough. In fact there exists $\eta>0$ so that $B(Z, \eta) \cap \Omega_{\infty}^{c}=\emptyset$. Since $\Omega_{i}^{c} \rightarrow \Omega_{\infty}^{c}$, then for $i$ large enough $B\left(Z, \frac{\eta}{2}\right) \subset \Omega_{i}$. Let $P_{i} \in \partial \Omega_{i}$ so that

$$
\begin{aligned}
\left|Z-P_{i}\right| & =d\left(Z, \partial \Omega_{i}\right)=\eta_{i}, \\
\eta & \leqslant \eta_{i} \leqslant|Z| \text { and }\left|P_{i}\right| \leqslant 2|Z| .
\end{aligned}
$$

Let $N>1$ be a large constant, we first study

$$
\begin{aligned}
\int_{\partial \Omega_{i} \cap\left\{\left|Q-P_{i}\right|>N \eta_{i}\right\}} h_{i}(Q) d \omega_{i}^{Z}(Q)= & \int_{\partial \Omega_{i} \cap\left\{\left|Q-P_{i}\right|>N \eta_{i}\right\}} h_{i}(Q) k_{i}^{Z}(Q) d \sigma_{i}(Q) \\
= & \sum_{j=0}^{\infty} \int_{\partial \Omega_{i} \cap\left\{2^{j} N \eta_{i}<\left|P_{i}-Q\right| \leqslant 2^{j+1} N \eta_{i}\right\}} h_{i}(Q) k_{i}^{Z}(Q) d \sigma_{i}(Q) \\
\leqslant & \sum_{j=0}^{\infty}\left(\int_{\partial \Omega_{i} \cap\left\{2^{j} N \eta_{i}<\left|P_{i}-Q\right| \leqslant 2^{j+1} N \eta_{i}\right\}} h_{i}^{2}(Q) d \sigma_{i}(Q)\right)^{\frac{1}{2}} \\
& \times\left(\int_{\partial \Omega_{i} \cap\left\{2^{j} N \eta_{i}<\left|P_{i}-Q\right| \leqslant 2^{j+1} N \eta_{i}\right\}}\left(k_{i}^{Z}(Q)\right)^{2} d \sigma_{i}(Q)\right)^{\frac{1}{2}} .
\end{aligned}
$$

Here $k_{i}^{Z}(Q)$ denotes the Poisson kernel of $\Omega_{i}$ with pole at $Z$, and $d \omega_{i}^{Z}=k_{i}^{Z} d \sigma_{i}$. We look at each term separately. Note that since $\Omega$ is a $\delta$-Reifenberg flat chord arc domain so is $\Omega_{i}$. Moreover the fact that $\log h \in \operatorname{VMO}(\partial \Omega)$ implies that $\log h_{i} \in \operatorname{VMO}\left(\partial \Omega_{i}\right)$. Also (3.1) ensures

$$
\begin{aligned}
& \left(\int_{\partial \Omega_{i} \cap\left\{2^{j} N \eta_{i}<\left|P_{i}-Q\right| \leqslant 2^{j+1} N \eta_{i}\right\}} h_{i}^{2}(Q) d \sigma_{i}(Q)\right)^{\frac{1}{2}} \\
& \leqslant\left(\int_{\partial \Omega_{i} \cap\left\{\left|P_{i}-Q\right| \leqslant 2^{j+1} N \eta_{i}\right\}} h_{i}^{2}(Q) d \sigma_{i}(Q)\right)^{\frac{1}{2}} \\
& \leqslant \frac{C}{2^{j n / 2} N^{n / 2} \eta_{i}^{n / 2}} \partial \int_{\partial \Omega_{i} \cap\left\{\left|P_{i}-Q\right| \leqslant 2^{j+1} N \eta_{i}\right\}} h_{i}(Q) d \sigma_{i} \\
& \leqslant \frac{C}{2^{j n / 2} N^{n / 2} \eta_{i}^{n / 2}} \partial \Omega_{i} \cap\left\{|Q|<2^{j+1} N \eta_{i}+2|Z|\right\} \\
& \leqslant \frac{C}{2^{j n / 2} N^{n / 2} \eta_{i}^{n / 2}}\left(2^{j+1} N \eta_{i}+2|Z|\right)^{n\left(1+\varepsilon^{\prime}\right)} \\
& \leqslant \frac{C}{2^{j n / 2} N^{n / 2} \eta^{n / 2}}|Z|^{n\left(1+\varepsilon^{\prime}\right)}\left(2^{j} N\right)^{n\left(1+\varepsilon^{\prime}\right)} \\
& \leqslant C \eta^{-n / 2}|Z|^{n\left(1+\varepsilon^{\prime}\right)} 2^{j n\left(\frac{1}{2}+\varepsilon^{\prime}\right)} N^{n\left(\frac{1}{2}+\varepsilon\right)},
\end{aligned}
$$


where we have used (4.51) and (4.54) in several occasions with $\varepsilon^{\prime}$ instead of $\varepsilon\left(\varepsilon^{\prime}=\varepsilon^{\prime}(\varepsilon)\right)$. The constant $C$ above depends on $n, \varepsilon^{\prime}$ and the Ahlfors regularity constant.

We now look at the second term. If $Q \in\left\{2^{j} N \eta_{i}<\left|P_{i}-Q\right| \leqslant 2^{j+1} \eta_{i} N\right\}$,

$$
|Q-Z| \geqslant\left|Q-P_{i}\right|-\left|P_{i}-Z\right|>2^{j} \eta_{i} N-\eta_{i}>2^{j-1} \eta_{i} N
$$

Let $\rho_{j}>0$ be such that $2 N_{0} \rho_{j}=2^{j-1} \eta_{i} N$ where $N_{0}$ is as in Lemma 2.3. Cover

$$
\partial \Omega_{i} \cap\left\{2^{j} \eta_{i} N<\left|P_{i}-Q\right| \leqslant 2^{j+1} \eta_{i} N\right\}
$$

by balls $B\left(X_{l}, \rho_{j}\right)$, with

$$
X_{l} \in \partial \Omega_{i} \cap\left\{2^{j} \eta_{i} N<\left|P_{i}-Q\right| \leqslant 2^{j+1} \eta_{i} N\right\}
$$

so that $\left|X_{l}-X_{k}\right| \geqslant \frac{1}{2} \rho_{j}$ if $l \neq k$. Note that $\left\{B\left(X_{l}, \frac{\rho_{j}}{5}\right)\right\}_{l \geqslant 1}$ is a disjoint collection of balls. Note that $Z \in \Omega_{i} \backslash B\left(2 N_{0} \rho_{j}, X_{l}\right)$ for each $l$, thus since $\log h_{i} \in \operatorname{VMO}(\partial \Omega)$ by (2.33) we have that

$$
\left(\oint_{B\left(X_{l}, \rho_{j}\right)}\left(k_{i}^{Z}\right)^{2} d \sigma_{i}\right)^{\frac{1}{2}} \leqslant C \oint_{B\left(X_{l}, \rho_{j}\right)} k_{i}^{Z} d \sigma_{i}
$$

and

$$
\begin{gathered}
\int_{\partial \Omega_{i} \cap\left\{2^{j} \eta_{i} N<\left|P_{i}-Q\right| \leqslant 2^{j+1} \eta_{i} N\right\}}\left(k_{i}^{Z}\right)^{2} d \sigma_{i} \\
\leqslant \sum_{l} \int_{B\left(X_{l}, \rho_{j}\right)}\left(k_{i}^{Z}\right)^{2} d \sigma_{i} \leqslant C \sum_{l} \frac{1}{\sigma_{i}\left(B\left(X_{l}, \rho_{j}\right)\right)}\left(\int_{B\left(X_{l}, \rho_{j}\right)} k_{i}^{Z} d \sigma_{i}\right)^{2} \\
\leqslant C \sum_{l} \frac{r_{i}^{n}}{\sigma\left(B\left(r_{i} X_{l}+Q_{i}, \rho_{j} r_{i}\right)\right)}\left(\int_{B\left(X_{l}, \rho_{j}\right)} k_{i}^{Z} d \sigma_{i}\right)^{2} \leqslant \frac{C}{\rho_{j}^{n}} \sum_{l}\left(\int_{B\left(X_{l}, \rho_{j}\right)} k_{i}^{Z} d \sigma_{i}\right)^{2},
\end{gathered}
$$

where we have also used the fact that $\partial \Omega$ is Ahlfors regular. Since $\omega_{i}^{Z}$ is a doubling measure (with uniform constants on $i$, that only depend on the NTA character of $\Omega$ ) and $\rho_{j}=\frac{2^{j-1} \eta_{i} N}{2 N_{0}}$, (4.58) yields

$$
\begin{aligned}
& \left(\int_{\partial \Omega_{i} \cap\left\{2^{j} \eta_{i} N<\left|P_{i}-Q\right| \leqslant 2^{j+1} \eta_{i} N\right\}}\left(k_{i}^{Z}\right)^{2} d \sigma_{i}\right)^{\frac{1}{2}} \\
& \leqslant \frac{C}{\rho_{j}^{n / 2}} \sum_{l} \int_{B\left(X_{l}, \rho_{j}\right)} k_{i}^{Z} d \sigma_{i} \leqslant \frac{C}{\rho_{j}^{n / 2}} \sum_{l} \int_{B\left(X_{l}, \frac{\rho_{j}}{5}\right)} k_{i}^{Z} d \sigma_{i} \\
& \leqslant \frac{C}{\eta_{i}^{n / 2} N^{n / 2} 2^{j n / 2}} \partial \Omega_{i} \cap\left\{2^{j} \eta_{i} N-\frac{\rho_{j}}{5}<\left|P_{i}-Q\right| \leqslant 2^{j+1} \eta_{i} N+\frac{\rho_{j}}{5}\right\} \\
& \leqslant \frac{C}{\eta_{i}^{n / 2} N^{n / 2} 2^{j n / 2}} \omega_{i}^{Z}\left(\bar{B}\left(P_{i}, 2^{j+1} \eta_{i} N+\frac{\rho_{j}}{5}\right) \backslash \bar{B}\left(P_{i}, 2^{j} \eta_{i} N-\frac{\rho_{j}}{5}\right)\right)
\end{aligned}
$$

$4^{\mathrm{e}}$ SÉRIE - TOME $36-2003-\mathrm{N}^{\circ} 3$ 
Since $\left|Z-P_{i}\right|=\eta_{i} \geqslant \eta$, and $\omega_{i}^{X}\left(\bar{B}\left(P_{i}, 2^{j+1} \eta_{i} N+\frac{\rho_{j}}{5}\right) \backslash \bar{B}\left(P_{i}, 2^{j} \eta_{i} N-\frac{\rho_{j}}{5}\right)\right)$ is a non-negative harmonic function in $\Omega_{i}$ which vanishes on $B\left(P_{i}, 2^{j} \eta_{i} N-\frac{\rho_{j}}{5}\right) \cap \partial \Omega_{i}$, Corollaries 2.2 and 2.3 in Section 2 and Lemmas 4.9, 4.11 and 4.8 in [14] imply that

$$
\begin{aligned}
& \left(\int_{\partial \Omega_{i} \cap\left\{2^{j} \eta_{i} N<\left|P_{i}-Q\right| \leqslant 2^{j+1} \eta_{i} N\right\}}\left(k_{i}^{Z}\right)^{2} d \sigma_{i}\right)^{\frac{1}{2}} \\
& \leqslant \frac{C}{\eta^{n / 2} N^{n / 2} 2^{j n / 2}}\left(\frac{\left|Z-P_{i}\right|}{2^{j} N \eta_{i}}\right)^{\frac{3}{4}} \omega_{i}^{A\left(P_{i}, 2^{j-1} \eta_{i} N\right)}\left(B\left(P_{i}, 2^{j+1} \eta_{i} N+\frac{\rho_{j}}{5}\right)\right) \\
& \leqslant \frac{C}{\eta^{n / 2} N^{n / 2} 2^{j n / 2}} \frac{1}{2^{3 j / 4} N^{3 / 4}} \omega_{i}^{A\left(P_{i}, 2^{j-1} \eta_{i} N\right)}\left(B\left(P_{i}, 2^{j-2} \eta_{i} N\right)\right) \\
& \leqslant \frac{C}{\eta^{n / 2} N^{n / 2+3 / 4} 2^{j(n / 2+3 / 4)}}\left(2^{j-2} \eta_{i} N\right)^{n-1} G_{i}\left(A\left(P_{i}, 2^{j-1} \eta_{i} N\right), A\left(P_{i}, 2^{j-2} \eta_{i} N\right)\right) \\
& \leqslant \frac{C}{\eta^{n / 2} N^{n / 2+3 / 4} 2^{j(n / 2+3 / 4)}}
\end{aligned}
$$

Combining (4.55), (4.56) and (4.60) we obtain

$$
\begin{aligned}
\int_{\partial \Omega_{i} \cap\left\{\left|Q-P_{i}\right|>N \eta_{i}\right\}} h_{i}(Q) d \omega_{i}^{Z}(Q) & \leqslant C \eta^{-n}|Z|^{n\left(1+\varepsilon^{\prime}\right)} N^{-\frac{3}{4}+n \varepsilon^{\prime}} \sum_{j=0}^{\infty}\left(2^{-\frac{3}{4}+n \varepsilon^{\prime}}\right)^{j} \\
& \leqslant C\left(n, \varepsilon^{\prime}, \eta,|Z|\right) N^{-\frac{3}{4}+n \varepsilon^{\prime}} \sum_{j=0}^{\infty}\left(2^{-\frac{3}{4}+n \varepsilon^{\prime}}\right)^{j} .
\end{aligned}
$$

Choosing $\varepsilon^{\prime}>0$ so that $n \varepsilon^{\prime}<\frac{1}{4}, N>4$ large enough so that

$$
C\left(n, \varepsilon^{\prime}, \eta,|Z|\right) N^{-\frac{1}{2}} \sum_{j=0}^{\infty} 2^{-\frac{1}{2} j}<\varepsilon \quad \text { and } \quad M>2 N|Z|
$$

we conclude using (4.54) that for $i \geqslant i_{0}$, since $|Q|>M$ implies

$$
\left|Q-P_{i}\right|>M-2|Z| \geqslant N \eta_{i}
$$

then

$$
\int_{\partial \Omega_{i} n\{|Q|>M\}} h_{i}(Q) d \omega_{i}^{Z}(Q) \leqslant \int_{\partial \Omega_{i} \cap\left\{\left|Q-P_{i}\right| \geqslant N \eta_{i}\right\}} h_{i} d \omega_{i}^{Z}<\varepsilon .
$$

Lemma 4.3. - Let $Z \in \Omega_{\infty}$. Then

$$
\limsup _{i \rightarrow \infty} \int_{\partial \Omega_{i}} h_{i} d \omega_{i}^{Z} \leqslant 1
$$

Proof. - Let $\varepsilon>0$, choose $i_{0} \geqslant 1$ and $M$ as in Lemma 4.2, in particular $|Z|<\frac{M}{8}$ and (4.53) holds. We concentrate on the quantity $\int_{\partial \Omega_{i} \cap B(0, M)} h_{i} d \omega_{i}^{Z}$. We use the following result which follows from the fact that $0 \in \partial \Omega$ and $\log h \in \operatorname{VMO}(\partial \Omega)$ : given $\varepsilon^{\prime}>0$ there exists $r\left(\varepsilon^{\prime}\right)>0$ such that for $r \in\left(0, r\left(\varepsilon^{\prime}\right)\right)$ and $Q \in B(0,1) \cap \partial \Omega$ there exists $G(Q, r) \subset B(Q, r) \cap \partial \Omega$ such that 
$\sigma(B(Q, r)) \leqslant\left(1+\varepsilon^{\prime}\right) \sigma(G(Q, r))$ and for all $P \in G(Q, r)$

$$
\left(1+\varepsilon^{\prime}\right)^{-1} \oint_{B(Q, r)} h d \sigma \leqslant h(P) \leqslant\left(1+\varepsilon^{\prime}\right) \oint_{B(Q, r)} h d \sigma .
$$

For further details see Lemma 5.6 in [18] and its proof. Although Lemma 5.6 in [18] is stated for chord arc domains with small constant the reader can easily check that the argument presented there only uses the fact that the domain is a Reifenberg flat chord arc domain. It is essentially a consequence of the definition of VMO and the John-Nirenberg inequality.

For $\varepsilon^{\prime}>0$ to be chosen depending on $\varepsilon$ and $M$, let $i_{1} \geqslant i_{0}$ so that for $i \geqslant i_{1}, M r_{i}<r\left(\varepsilon^{\prime}\right)$, and $\left|Q_{i}\right|<1$ (recall that $Q_{i} \rightarrow 0$ ). Let $G_{i}=\frac{1}{r_{i}}\left(G\left(Q_{i}, M r_{i}\right)-Q_{i}\right)$, and $F_{i}=\partial \Omega_{i} \cap B(0, M) \backslash G_{i}$, where $G\left(Q_{i}, M r_{i}\right) \subset B\left(Q_{i}, M r_{i}\right) \cap \partial \Omega$ satisfies

$$
\sigma\left(B\left(Q_{i}, M r_{i}\right)\right) \leqslant\left(1+\varepsilon^{\prime}\right) \sigma\left(G\left(Q_{i}, M r_{i}\right)\right)
$$

and for all $P \in G\left(Q_{i}, M r_{i}\right)$

$$
\int_{B\left(Q_{i}, M r_{i}\right)} h d \sigma \underset{\varepsilon^{\prime}}{\sim} h(P),
$$

where $a \underset{\varepsilon^{\prime}}{\sim} b$ means that $\frac{1}{1+\varepsilon^{\prime}} \leqslant \frac{a}{b} \leqslant 1+\varepsilon^{\prime}$. We split the integral above in 2 parts

$$
\int_{\partial \Omega_{i} \cap B(0, M)} h_{i} d \omega_{i}^{Z}=\int_{G_{i}} h_{i} d \omega_{i}^{Z}+\int_{F_{i}} h_{i} d \omega_{i}^{Z}
$$

For $Q \in G_{i}, r_{i} Q+Q_{i} \in G\left(Q_{i}, M r_{i}\right)$, the definition of $h_{i}$ and (4.66) yield

$$
h_{i}(Q)=\frac{h\left(r_{i} Q+Q_{i}\right)}{f_{B\left(Q_{i}, r_{i}\right)} h d \sigma} \underset{\varepsilon^{\prime}}{\sim} \frac{f_{B\left(Q_{i}, M r_{i}\right)} h d \sigma}{f_{B\left(Q_{i}, r_{i}\right)} h d \sigma},
$$

which implies that

$$
\int_{G_{i}} h_{i} d \omega_{i}^{Z} \leqslant\left(1+\varepsilon^{\prime}\right) \frac{f_{B\left(Q_{i}, M r_{i}\right)} h d \sigma}{\oint_{B\left(Q_{i}, r_{i}\right)} h d \sigma} \omega_{i}^{Z}\left(G_{i}\right) \leqslant\left(1+\varepsilon^{\prime}\right) \frac{\oint_{B\left(Q_{i}, M r_{i}\right)} h d \sigma}{\oint_{B\left(Q_{i}, r_{i}\right)} h d \sigma}
$$

because $\omega_{i}^{Z}$ is a probability measure. Note that

$$
\begin{aligned}
\int_{B\left(Q_{i}, r_{i}\right)} h d \sigma & \geqslant \frac{1}{\sigma\left(B\left(Q_{i}, r_{i}\right)\right)} \int_{B\left(Q_{i}, r_{i}\right) \cap G\left(Q_{i}, M r_{i}\right)} h d \sigma \\
& \geqslant \frac{1}{\left(1+\varepsilon^{\prime}\right)} \frac{\sigma\left(B\left(Q_{i}, r_{i}\right) \cap G\left(Q_{i}, M r_{i}\right)\right)}{\sigma\left(B\left(Q_{i}, r_{i}\right)\right)} \oint_{B\left(Q_{i}, M r_{i}\right)} h d \sigma .
\end{aligned}
$$

Combining (4.69) and (4.70) we obtain

$$
\int_{G_{i}} h_{i} d \omega_{i}^{Z} \leqslant\left(1+\varepsilon^{\prime}\right)^{2} \frac{\sigma\left(B\left(Q_{i}, r_{i}\right)\right)}{\sigma\left(B\left(Q_{i}, r_{i}\right) \cap G\left(Q_{i}, M r_{i}\right)\right)} .
$$


Moreover since $\partial \Omega$ is Ahlfors regular

$$
\begin{aligned}
\sigma\left(B\left(Q_{i}, r_{i}\right) \cap G\left(Q_{i}, M r_{i}\right)\right) & =\sigma\left(B\left(Q_{i}, r_{i}\right)\right)-\sigma\left(B\left(Q_{i}, r_{i}\right) \backslash G\left(Q_{i}, M r_{i}\right)\right) \\
& \geqslant \sigma\left(B\left(Q_{i}, r_{i}\right)\right)-\sigma\left(B\left(Q_{i}, M r_{i}\right) \backslash G\left(Q_{i}, M r_{i}\right)\right) \\
& \geqslant \sigma\left(B\left(Q_{i}, r_{i}\right)\right)-\varepsilon^{\prime} \sigma\left(B\left(Q_{i}, M r_{i}\right)\right) \\
& \geqslant \sigma\left(B\left(Q_{i}, r_{i}\right)\right)\left(1-C M^{n} \varepsilon^{\prime}\right) .
\end{aligned}
$$

Combining (4.71) and (4.72) we have

$$
\int_{G_{i}} h_{i} d \omega_{i}^{Z} \leqslant\left(1+\varepsilon^{\prime}\right)^{2}\left(1-C M^{n} \varepsilon^{\prime}\right)^{-1} .
$$

We estimate now the second term in (4.67). Since $Z \in B\left(0, \frac{M}{8}\right)$, and $\Omega_{i}$ is a $\delta$-Reifenberg flat chord arc domain, there exists $C=C(|Z|, \eta, M)$ so that if $A_{i}=A\left(0,16 N_{0} M\right) \in \Omega_{i}$ is a nontangential point for $\Omega_{i}$, at 0 and radius $16 M N_{0}$, with $N_{0}$ as in Lemma 2.3 then by the boundary Harnack principle

$$
\begin{aligned}
\int_{F_{i}} h_{i} d \omega_{i}^{Z} & \leqslant C \int_{F_{i}} h_{i} d \omega_{i}^{A_{i}}=C \int_{F_{i}} h_{i} k_{i}^{A_{i}} d \sigma_{i} \\
& \leqslant C\left(\int_{F_{i}} h_{i}^{2} d \sigma_{i}\right)^{\frac{1}{2}}\left(\int_{F_{i}}\left(k_{i}^{A_{i}}\right)^{2} d \sigma_{i}\right)^{\frac{1}{2}} .
\end{aligned}
$$

Note that

$$
\int_{F_{i}} h_{i}^{2} d \sigma_{i}=r_{i}^{-n} \frac{\int_{B\left(Q_{i}, M r_{i}\right) \backslash G\left(Q_{i}, M r_{i}\right)} h^{2} d \sigma}{\left(\oint_{B\left(Q_{i}, r_{i}\right)} h d \sigma\right)^{2}} .
$$

Since $\log h^{2} \in \operatorname{VMO}(\partial \Omega)$ for $i$ large enough

$$
\begin{gathered}
\int_{B\left(Q_{i}, M r_{i}\right) \backslash G\left(Q_{i}, M r_{i}\right)} h^{2} d \sigma \\
\leqslant C\left(\frac{\sigma\left(B\left(Q_{i}, M r_{i}\right) \backslash G\left(Q_{i}, M r_{i}\right)\right)}{\sigma\left(B\left(Q_{i}, M r_{i}\right)\right)}\right)^{\frac{1}{2}} \int_{B\left(Q_{i}, M r_{i}\right)} h^{2} d \sigma \\
\leqslant C\left(\varepsilon^{\prime}\right)^{\frac{1}{2}} \int_{B\left(Q_{i}, M r_{i}\right)} h^{2} d \sigma \\
\leqslant C \sqrt{\varepsilon^{\prime}} \sigma\left(B\left(Q_{i}, M r_{i}\right)\right)\left(f_{B\left(Q_{i}, M r_{i}\right)} h d \sigma\right)^{2} .
\end{gathered}
$$

Combining (4.75), (4.76), (4.70) and (4.72) we obtain

$$
\begin{aligned}
\int_{F_{i}} h_{i}^{2} d \sigma_{i} & \leqslant C \sqrt{\varepsilon^{\prime}} \frac{\sigma\left(B\left(Q_{i}, M r_{i}\right)\right)}{r_{i}^{n}} \cdot \frac{\left(\oint_{B\left(Q_{i}, M r_{i}\right)} h d \sigma\right)^{2}}{\left(\oint_{B\left(Q_{i}, r_{i}\right)} h d \sigma\right)^{2}} \\
& \leqslant C \sqrt{\varepsilon^{\prime}} M^{n}\left(1-C M^{n} \varepsilon^{\prime}\right)^{-2}
\end{aligned}
$$


provided that $\varepsilon^{\prime}<1$ and small enough. Since $\partial \Omega_{i}$ is a $\delta$-Reifenberg flat chord arc domain, $\log h_{i} \in \operatorname{VMO}\left(\partial \Omega_{i}\right)$, and $\left\|\log h_{i}\right\|_{*}\left(\partial \Omega_{i}\right) \sim\|\log h\|_{*}(\partial \Omega)$, (2.32) is satisfied, and hence by Lemma 2.3 we have that

$$
\begin{aligned}
\left(\int_{F_{i}}\left(k_{i}^{A_{i}}\right)^{2} d \sigma_{i}\right)^{\frac{1}{2}} & \leqslant\left(\int_{B(0, M) \cap \partial \Omega_{i}}\left(k_{i}^{A_{i}}\right)^{2} d \sigma_{i}\right)^{\frac{1}{2}} \\
& \leqslant C \sigma_{i}(B(0, M))^{\frac{1}{2}}\left(\oint_{B(0, M) \cap \partial \Omega_{i}} k_{i}^{A_{i}} d \sigma_{i}\right) \\
& \leqslant C \sigma_{i}(B(0, M))^{-\frac{1}{2}} \omega^{A_{i}}(B(0, M)) \\
& \leqslant C\left(\frac{\sigma\left(B\left(Q_{i}, M r_{i}\right)\right)}{r_{i}^{n}}\right)^{-\frac{1}{2}} \leqslant C M^{-\frac{n}{2}}
\end{aligned}
$$

Combining (4.74), (4.78) and (4.79) we obtain that if $C M^{n} \varepsilon^{\prime}<\frac{1}{2}$ then

$$
\int_{F_{i}} h_{i} d \omega_{i}^{Z} \leqslant C_{M}\left(\varepsilon^{\prime}\right)^{\frac{1}{4}}
$$

Inequalities (4.73) and (4.80) yield

$$
\int_{\partial \Omega_{i} \cap B(0, M)} h_{i} d \omega_{i}^{Z} \leqslant\left(1+\varepsilon^{\prime}\right)^{2}\left(1-C M^{n} \varepsilon^{\prime}\right)^{-1}+C_{M}\left(\varepsilon^{\prime}\right)^{\frac{1}{4}} .
$$

Choosing $\varepsilon^{\prime}>0$ so that $C_{M}\left(\varepsilon^{\prime}\right)^{\frac{1}{4}}<\frac{\varepsilon}{2}$ and $\left(1+\varepsilon^{\prime}\right)^{2}\left(1-C M^{n} \varepsilon^{\prime}\right)^{-1}<1+\frac{\varepsilon}{2}$, recalling our choice of $M$, and combining (4.53) and (4.81) we obtain that for $\varepsilon>0$ there exists $i_{\varepsilon} \geqslant 1$ so that

$$
\sup _{i \geqslant i_{\varepsilon}} \int_{\partial \Omega_{i}} h_{i} d \omega_{i}^{Z} \leqslant 1+2 \varepsilon
$$

therefore

$$
\limsup _{i \rightarrow \infty} \int_{\partial \Omega_{i}} h_{i} d \omega_{i}^{Z} \leqslant 1
$$

We are now ready to finish the proof of Theorem 4.2.

Proof of Theorem 4.2. - Let $Z \in \Omega_{\infty}$, let $i_{0} \geqslant 1$ so that $\eta=\inf _{i \geqslant i_{0}} d\left(Z, \partial \Omega_{i}\right)>0$, by (4.8) $u_{i} \rightarrow u_{\infty}$ uniformly on $B\left(Z, \frac{\eta}{2}\right)$, thus by harmonicity $\nabla u_{i} \rightarrow \nabla u_{\infty}$ uniformly on $B\left(Z, \frac{\eta}{4}\right)$. Thus $\lim _{i \rightarrow \infty}\left|\nabla u_{i}(Z)\right|=\left|\nabla u_{\infty}(Z)\right|$. Now we consider two different cases: either $u$ denotes the Green's function with finite pole $A$ or $u$ denotes the Green's function with pole at infinity. In the second case $u_{i}$ denotes the Green's function of $\Omega_{i}$ with pole at infinity with corresponding Poisson kernel $h_{i}$. By Theorem 3.1

$$
\left|\nabla u_{i}(Z)\right| \leqslant \int_{\partial \Omega_{i}} h_{i} d \omega_{i}^{Z}
$$


Thus by Lemma 4.3 we have that

$$
\left|\nabla u_{\infty}(Z)\right| \leqslant 1
$$

If $u$ denotes the Green's function of $\Omega$ with pole at $A, u_{i}$ is a multiple of the Green's function of $\Omega_{i}$ with pole at $A_{i}=\frac{A-Q_{i}}{r_{i}}$. In fact by (4.2) and (4.43) we have that

$$
u_{i}(Z)=\frac{\sigma\left(B\left(Q_{i}, r_{i}\right)\right)}{r_{i}^{n}} \frac{G_{i}\left(A_{i}, Z\right)}{\omega_{i}^{A_{i}}(B(0,1))}
$$

Since for $i$ large $\delta_{i}(Z)=\operatorname{dist}\left(Z, \partial \Omega_{i}\right)<\frac{\delta_{i}\left(A_{i}\right)}{4}=\frac{1}{4 r_{i}} \delta(A)$, and $Z \in B\left(0, \frac{\delta_{i}\left(A_{i}\right)}{4}\right)$ by Theorem 3.2 we have that

$$
\left|\nabla G_{i}\left(A_{i}, Z\right)\right| \leqslant \int_{\partial \Omega_{i}} k_{i}^{A_{i}} d \omega_{i}^{z}+C \frac{\omega_{i}^{A_{i}}\left(B\left(0, \delta_{i}\left(A_{i}\right)\right)\right)}{\left(\delta_{i}(A)\right)^{n}}\left(\frac{\delta_{i}(Z)}{\delta_{i}\left(A_{i}\right)}\right)^{\frac{3}{4}} .
$$

Combining (4.86), (4.87), (4.13) and (4.39), and using the fact that $\partial \Omega$ is Ahlfors regular we obtain

$$
\left|\nabla u_{i}(Z)\right| \leqslant \int_{\partial \Omega_{i}} h_{i} d \omega_{i}^{Z}+C \frac{\omega^{A}\left(B\left(Q_{i}, \delta(A)\right)\right)}{\delta(A)^{n}} \frac{r_{i}^{n}}{\omega^{A}\left(B\left(Q_{i}, r_{i}\right)\right.}\left(\frac{\delta_{i}(Z)}{\delta_{i}\left(A_{i}\right)}\right)^{\frac{3}{4}}
$$

Since $h=k_{A}$, and $\log h \in \operatorname{VMO}(\partial \Omega)$, Corollary 2.4 ensures that

$$
\frac{\omega^{A}\left(B\left(Q_{i}, \delta(A)\right)\right)}{\omega^{A}\left(B\left(Q_{i}, r_{i}\right)\right)} \leqslant C\left(\frac{\sigma\left(B\left(Q_{i}, \delta(A)\right)\right)}{\sigma\left(B\left(Q_{i}, r_{i}\right)\right)}\right)^{1+\frac{1}{4 n}} \leqslant C\left(\frac{\delta(A)}{r_{i}}\right)^{n+\frac{1}{8}} .
$$

Combining (4.88) and (4.89) we obtain for $i$ large enough that

$$
\begin{aligned}
\left|\nabla u_{i}(Z)\right| & \leqslant \int_{\partial \Omega_{i}} h_{i} d \omega_{i}^{Z}+C\left(\frac{\delta(A)}{r_{i}}\right)^{\frac{1}{8}}\left(\frac{r_{i}}{\delta(A)}\right)^{\frac{3}{4}}\left(\delta_{\infty}(Z)\right)^{\frac{3}{4}} \\
& \leqslant \int_{\partial \Omega_{i}} h_{i} d \omega_{i}^{Z}+C\left(\frac{r_{i}}{\delta(A)}\right)^{\frac{1}{2}}\left(\delta_{\infty}(Z)\right)^{\frac{3}{4}}
\end{aligned}
$$

where $\delta_{\infty}(Z)=\operatorname{dist}\left(Z, \partial \Omega_{\infty}\right)$. Thus by (4.63) letting $i$ tend to infinity in (4.90) we have that (4.10) also holds in this case. This concludes the proof of Theorem 4.2.

THEOREM 4.3. - If $\Omega_{\infty} \subset \mathbb{R}^{n+1}, u_{\infty}$ and $\omega_{\infty}$ are as in Theorem 4.1 , then $h_{\infty}=\frac{d \omega_{\infty}}{d \sigma_{\infty}}$ satisfies

$$
h_{\infty}(Q) \geqslant 1 \text { for } \mathcal{H}^{n} \text {-a.e. } Q \in \partial \Omega_{\infty} .
$$

Proof. - By Theorem $4.1 \Omega_{\infty}$ is an unbounded $4 \delta$-Reifenberg flat chord arc domain. Hence $\omega_{\infty}$ and $\sigma_{\infty}$ are mutually absolutely continuous (see again [4] and [25]), and the RadonNikodym theorem ensures that $h_{\infty}=\frac{d \omega_{\infty}}{d \sigma_{\infty}} \in L_{\text {loc }}^{1}\left(d \sigma_{\infty}\right)$. Moreover for $\varphi \in C_{c}^{\infty}\left(\mathbb{R}^{n+1}\right)$

$$
\lim _{i \rightarrow \infty} \int_{\partial \Omega_{i}} \varphi h_{i} d \sigma_{i}=\lim _{i \rightarrow \infty} \int_{\partial \Omega_{i}} \varphi d \omega_{i}=\int_{\partial \Omega_{\infty}} \varphi d \omega_{\infty}=\int_{\partial \Omega_{\infty}} \varphi h_{\infty} d \sigma_{\infty} .
$$


Recall that each $\Omega_{i}$ is a $\delta$-Reifenberg flat chord arc domain, and that $\chi_{\Omega_{i}} \rightarrow \chi_{\Omega_{\infty}}$ in $L_{\text {loc }}^{1}\left(\mathbb{R}^{n+1}\right)$. Let $\overrightarrow{n_{i}}$ denote the inner unit normal to $\partial \Omega_{i}, \overrightarrow{n_{\infty}}$ denote the inner unit normal to $\partial \Omega_{\infty}$ and let $e \in \mathbb{S}^{n}$, then for $\varphi \in C_{c}^{\infty}\left(\mathbb{R}^{n+1}\right)$ and $\varphi \geqslant 0$,

$$
\int_{\partial \Omega_{i}} \varphi d \sigma_{i} \geqslant \int_{\partial \Omega_{i}} \varphi\left\langle\overrightarrow{n_{i}}, e\right\rangle d \sigma_{i}=-\int_{\Omega_{i}} \operatorname{div}(\varphi e)
$$

and since $\left\langle e, \overrightarrow{n_{\infty}}\right\rangle=\frac{1}{2}\left(|e|^{2}+\left|\overrightarrow{n_{\infty}}\right|^{2}-\left|\overrightarrow{n_{\infty}}-e\right|^{2}\right)=1-\frac{1}{2}\left|\overrightarrow{n_{\infty}}-e\right|^{2}$,

$$
\begin{aligned}
\liminf _{i \rightarrow \infty} \int_{\partial \Omega_{i}} \varphi d \sigma_{i} & \geqslant-\int_{\Omega_{\infty}} \operatorname{div}(\varphi e)=\int_{\partial \Omega_{\infty}} \varphi\left\langle e, \overrightarrow{n_{\infty}}\right\rangle d \sigma_{\infty} \\
& \geqslant \int_{\partial \Omega_{\infty}} \varphi d \sigma_{\infty}-\frac{1}{2} \int_{\partial \Omega_{\infty}} \varphi\left|\overrightarrow{n_{\infty}}-e\right|^{2} d \sigma_{\infty} .
\end{aligned}
$$

Assume that support $(\varphi) \subset B(0, M)$, and $\varphi \geqslant 0$. Using the same notation as in the proof of Theorem 4.2 we know that since $\log h \in \operatorname{VMO}(\partial \Omega)$, for $\varepsilon \in(0,1)$ there exists $i_{0} \geqslant 1$ so that for $i \geqslant i_{0}$ and $\left|Q_{i}\right|<1$ there exists $G\left(Q_{i}, M r_{i}\right) \subset B\left(Q_{i}, M r_{i}\right) \cap \partial \Omega$ satisfying

$$
\sigma\left(B\left(Q_{i}, M r_{i}\right)\right) \leqslant(1+\varepsilon) \sigma\left(G\left(Q_{i}, M r_{i}\right)\right)
$$

and

$$
\oint_{B\left(Q_{i}, M r_{i}\right)} h d \sigma \underset{\varepsilon}{\widetilde{c}} h(P) \quad \text { for } P \in G\left(Q_{i}, M r_{i}\right) .
$$

If $G_{i}=\frac{1}{r_{i}}\left(G\left(Q_{i}, M r_{i}\right)-Q_{i}\right)$ and $F_{i}=\partial \Omega_{i} \cap B(0, M) \backslash G_{i}$, then for $Q \in G_{i}$

$$
h_{i}(Q)=\frac{h\left(r_{i} Q+Q_{i}\right)}{\oint_{B\left(Q_{i}, r_{i}\right)} h d \sigma} \widetilde{\varepsilon} \frac{f_{B\left(Q_{i}, M r_{i}\right)} h d \sigma}{f_{B\left(Q_{i}, r_{i}\right)} h d \sigma}
$$

which implies that

$$
\int_{G_{i}} h_{i} \varphi d \sigma_{i} \underset{\varepsilon}{\widetilde{\varepsilon}} \frac{f_{B\left(Q_{i}, M r_{i}\right)} h d \sigma}{f_{B\left(Q_{i}, r_{i}\right)} h d \sigma} \int_{G_{i}} \varphi d \sigma_{i} .
$$

Moreover using the fact that $\partial \Omega$ is Ahlfors regular, the definition of $\sigma_{i}=\mathcal{H}^{n}\left\llcorner\partial \Omega_{i}\right.$ and (4.95) we have

$$
\begin{aligned}
\int_{G_{i}} \varphi d \sigma_{i} & =\int_{\partial \Omega_{i}} \varphi d \sigma_{i}-\int_{F_{i}} \varphi d \sigma_{i} \geqslant \int_{\partial \Omega_{i}} \varphi d \sigma_{i}-\|\varphi\|_{\infty} \sigma_{i}\left(F_{i}\right) \\
& \geqslant \int_{\partial \Omega_{i}} \varphi d \sigma_{i}-C\|\varphi\|_{\infty} M^{n} \varepsilon .
\end{aligned}
$$

Combining (4.98) and (4.99) we obtain for $i \geqslant i_{0}$

$$
\int_{\partial \Omega_{i}} \varphi d \sigma_{i} \leqslant \int_{G_{i}} \varphi d \sigma_{i}+C\|\varphi\|_{\infty} M^{n} \varepsilon
$$




$$
\begin{aligned}
& \leqslant(1+\varepsilon) \frac{\oint_{B\left(Q_{i}, r_{i}\right)} h d \sigma}{\oint_{B\left(Q_{i}, M r_{i}\right)} h d \sigma} \int_{G_{i}} h_{i} \varphi d \sigma_{i}+C M^{n}\|\varphi\|_{\infty} \varepsilon \\
& \leqslant(1+\varepsilon)^{2} \frac{\oint_{B\left(Q_{i}, r_{i}\right)} h d \sigma}{\oint_{B\left(Q_{i}, M r_{i}\right)} h d \sigma} \int_{\partial \Omega_{i}} \varphi d \omega_{i}+C M^{n}\|\varphi\|_{\infty} \varepsilon .
\end{aligned}
$$

Furthermore (4.95), (4.96), (2.4) and our choice of $\varepsilon=\frac{1}{4}$, the fact that $\log h \in \operatorname{VMO}(\partial \Omega)$ and $\partial \Omega$ is Ahlfors regular yield

$$
\begin{aligned}
\oint_{B\left(Q_{i}, r_{i}\right)} h d \sigma= & \frac{1}{\sigma\left(B\left(Q_{i}, r_{i}\right)\right)}\left\{\int_{B\left(Q_{i}, r_{i}\right) \cap G\left(Q_{i}, M r_{i}\right)} h d \sigma+\int_{B\left(Q_{i}, r_{i}\right) \cap G\left(Q_{i}, M r_{i}\right)^{c}} h d \sigma\right\} \\
\leqslant & (1+\varepsilon) \frac{\sigma\left(B\left(Q_{i}, r_{i}\right) \cap G\left(Q_{i}, M r_{i}\right)\right)}{\sigma\left(B\left(Q_{i}, r_{i}\right)\right)} \oint_{B\left(Q_{i}, M r_{i}\right)} h d \sigma \\
& +\frac{\omega\left(B\left(Q_{i}, r_{i}\right) \cap G\left(Q_{i}, M r_{i}\right)^{c}\right)}{\sigma\left(B\left(Q_{i}, r_{i}\right)\right)} \\
\leqslant & (1+\varepsilon) \oint_{B\left(Q_{i}, M r_{i}\right)} h d \sigma+C \frac{\omega\left(B\left(Q_{i}, r_{i}\right)\right)}{\sigma\left(B\left(Q_{i}, r_{i}\right)\right)}\left(\frac{\sigma\left(B\left(Q_{i}, r_{i}\right) \cap G\left(Q_{i}, M r_{i}\right)^{c}\right.}{\sigma\left(B\left(Q_{i}, r_{i}\right)\right)}\right)^{\frac{3}{4}} \\
\leqslant & \oint_{B\left(Q_{i}, M r_{i}\right)} h d \sigma+C \varepsilon^{\frac{3}{4}} M^{\frac{3 n}{4}} \oint_{B\left(Q_{i}, r_{i}\right)} h d \sigma,
\end{aligned}
$$

which implies

$$
\oint_{B\left(Q_{i}, r_{i}\right)} h d \sigma \leqslant\left(1+C \varepsilon^{\frac{3}{4}} M^{\frac{3 n}{4}}\right) \oint_{B\left(Q_{i}, M r_{i}\right)} h d \sigma .
$$

Combining (4.100) and (4.102) we have for $i \geqslant i_{0}$

$$
\int_{\partial \Omega_{i}} \varphi d \sigma_{i} \leqslant\left(1+C M^{\frac{5 n}{4}} \varepsilon^{\frac{1}{4}}\right) \int_{\partial \Omega_{i}} \varphi d \omega_{i}+C M^{n}\|\varphi\|_{\infty} \varepsilon .
$$

Thus (4.103) ensures that for every $\varepsilon>0$, and $\varphi \in C_{c}^{\infty}(B(0, M)), \varphi \geqslant 0$

$$
\begin{aligned}
\limsup _{i \rightarrow \infty} \int_{\partial \Omega_{i}} \varphi d \sigma_{i} & \leqslant\left(1+C M^{\frac{5 n}{4}} \varepsilon^{\frac{1}{4}}\right) \lim _{i \rightarrow \infty} \int_{\partial \Omega_{i}} \varphi d \omega_{i}+C M^{n}\|\varphi\|_{\infty} \varepsilon \\
& \leqslant\left(1+C M^{\frac{5 n}{4}} \varepsilon^{\frac{1}{4}}\right) \int_{\partial \Omega_{\infty}} \varphi d \omega_{\infty}+C M^{n}\|\varphi\|_{\infty} \varepsilon .
\end{aligned}
$$

Letting $\varepsilon \rightarrow 0$ we conclude that for $\varphi \in C_{c}^{\infty}\left(\mathbb{R}^{n+1}\right) \varphi \geqslant 0$

$$
\limsup _{i \rightarrow \infty} \int_{\partial \Omega_{i}} \varphi d \sigma_{i} \leqslant \int_{\partial \Omega_{\infty}} \varphi h_{\infty} d \sigma_{\infty}
$$


Combining (4.94) and (4.105) we have that for $\varphi \in C_{c}^{\infty}\left(\mathbb{R}^{n+1}\right), \varphi \geqslant 0$

$$
\int_{\partial \Omega_{\infty}} \varphi h_{\infty} d \sigma_{\infty} \geqslant \int_{\partial \Omega_{\infty}} \varphi d \sigma_{\infty}-\frac{1}{2} \int_{\partial \Omega_{\infty}} \varphi\left|\overrightarrow{n_{\infty}}-e\right|^{2} d \sigma_{\infty} .
$$

Let $Q \in \partial^{*} \Omega_{\infty}$, approximating $\chi_{B(Q, r)}$ by smooth functions with compact support, and letting $e=\overrightarrow{n_{\infty}}(Q),(4.106)$ implies that

$$
\int_{B(Q, r)} h_{\infty} d \sigma_{\infty} \geqslant \int_{B(Q, r)} d \sigma_{\infty}-\frac{1}{2} \int_{B(Q, r)}\left|\overrightarrow{n_{\infty}}-\overrightarrow{n_{\infty}}(Q)\right|^{2} d \sigma_{\infty}
$$

and

$$
\oint_{B(Q, r)} h_{\infty} d \sigma_{\infty} \geqslant 1-\frac{1}{2} \oint_{B(Q, r)}\left|\overrightarrow{n_{\infty}}-\overrightarrow{n_{\infty}}(Q)\right|^{2} d \sigma_{\infty} .
$$

Since $Q \in \partial^{*} \Omega_{\infty}, \lim _{r \rightarrow 0} \oint_{B(Q, r)}\left|\overrightarrow{n_{\infty}}-\overrightarrow{n_{\infty}}(Q)\right|^{2} d \sigma_{\infty}=0$, thus (4.108) implies that for $\mathcal{H}^{n}$ a.e. $Q \in \partial \Omega_{\infty}$

$$
h_{\infty}(Q)=\lim _{r \rightarrow 0} \int_{B(Q, r)} h_{\infty} d \sigma_{\infty} \geqslant 1 .
$$

THEOREM 4.4. - The subsequence introduced in 4.1 also satisfies

$$
\sigma_{i} \rightarrow \sigma_{\infty}
$$

weakly as Radon measures, where $\sigma_{\infty}=\mathcal{H}^{n}\left\llcorner\partial \Omega_{\infty}\right.$.

Proof. - Let $\varphi \in C_{c}^{\infty}\left(\mathbb{R}^{n+1}\right), \varphi \geqslant 0$ and suppose that $\operatorname{support}(\varphi) \subset \mathrm{B}(0, \mathrm{M})$. Using the same notation as in the proof of Theorem 4.3 we have that given $\varepsilon>0$ there exists $i_{0} \geqslant 1$ such that for $i \geqslant i_{0}($ see $(4.103))$

$$
\int_{\partial \Omega_{i}} \varphi d \sigma_{i} \leqslant\left(1+C M^{\frac{5 n}{4}} \varepsilon^{\frac{1}{4}}\right) \int_{\partial \Omega_{i}} \varphi d \omega_{i}+C M^{n}\|\varphi\|_{\infty} \varepsilon .
$$

Since $\varphi \geqslant 0,(4.97)$ yields

$$
\int_{\partial \Omega_{i}} \varphi d \sigma_{i} \geqslant \int_{G_{i}} \varphi d \sigma_{i} \geqslant(1+\varepsilon)^{-1} \frac{\oint_{B\left(Q_{i}, r_{i}\right)} h d \sigma}{f_{B\left(Q_{i}, M r_{i}\right)} h d \sigma} \int_{G_{i}} h_{i} \varphi d \sigma_{i} .
$$

Furthermore (4.95), (4.96), the fact that $\log h \in \operatorname{VMO}(\partial \Omega)$ and $\partial \Omega$ is Ahlfors regular yield as in (4.102) that

$$
\begin{aligned}
\oint_{B\left(Q_{i}, r_{i}\right)} h d \sigma & \geqslant \frac{1}{\sigma\left(B\left(Q_{i}, r_{i}\right)\right)} \int_{B\left(Q_{i}, r_{i}\right) \cap G\left(Q_{i}, M r_{i}\right)} h d \sigma \\
& \geqslant(1+\varepsilon)^{-1} \frac{\sigma\left(B\left(Q_{i}, r_{i}\right) \cap G\left(Q_{i}, M r_{i}\right)\right)}{\sigma\left(B\left(Q_{i}, r_{i}\right)\right)} \oint_{B\left(Q_{i}, M r_{i}\right)} h d \sigma
\end{aligned}
$$




$$
\begin{aligned}
& \geqslant(1+\varepsilon)^{-1} \oint_{B\left(Q_{i}, M r_{i}\right)} h d \sigma\left[1-\frac{\sigma\left(B\left(Q_{i}, r_{i}\right) \cap G\left(Q_{i}, M r_{i}\right)\right)^{c}}{\sigma\left(B\left(Q_{i}, r_{i}\right)\right)}\right] \\
& \geqslant(1+\varepsilon)^{-1} \oint_{B\left(Q_{i}, M r_{i}\right)} h d \sigma\left[1-C M^{n} \varepsilon\right] .
\end{aligned}
$$

To estimate the term

$$
\int_{G_{i}} h_{i} \varphi d \sigma_{i}=\int_{\partial \Omega_{i}} \varphi d \omega_{i}-\int_{F_{i}} \varphi d \omega_{i},
$$

we need to bound the second term on the right hand side. Using (4.13), our choice of $\delta>0$ combined with (2.23), (4.95), and the fact that $\partial \Omega$ is Ahlfors regular, we obtain

$$
\begin{aligned}
\int_{F_{i}} \varphi d \omega_{i} & \leqslant\|\varphi\|_{\infty} \omega_{i}\left(F_{i}\right) \\
& \leqslant\|\varphi\|_{\infty} r_{i}^{-n} \sigma\left(B\left(Q_{i}, r_{i}\right)\right) \frac{\omega\left(G\left(Q_{i}, M r_{i}\right)^{c} \cap B\left(Q_{i}, M r_{i}\right)\right)}{\omega\left(B\left(Q_{i}, r_{i}\right)\right)} \\
& \leqslant C\|\varphi\|_{\infty} r_{i}^{-n} \sigma\left(B\left(Q_{i}, r_{i}\right)\right) \frac{\omega\left(B\left(Q_{i}, M r_{i}\right)\right)}{\omega\left(B\left(Q_{i}, r_{i}\right)\right)}\left(\frac{\sigma\left(G\left(Q_{i}, M r_{i}\right)^{c} \cap B\left(Q_{i}, M r_{i}\right)\right)}{\sigma\left(B\left(Q_{i}, r_{i}\right)\right)}\right)^{\frac{3}{4}} \\
(4.115) & \leqslant C\|\varphi\|_{\infty} M^{\frac{5 n}{4}} \varepsilon^{\frac{3}{4}} \frac{f_{B\left(Q_{i}, M r_{i}\right)} h d \sigma}{f_{B\left(Q_{i}, r_{i}\right)} h d \sigma} .
\end{aligned}
$$

Combining (4.112), (4.113), (4.114), and (4.115) we have for $\varepsilon>0$ small

$$
\int_{\partial \Omega_{i}} \varphi d \sigma_{i} \geqslant\left(1-C \varepsilon M^{n}\right) \int_{\partial \Omega_{i}} \varphi d \omega_{i}-C\|\varphi\|_{\infty} M^{n} \varepsilon^{\frac{1}{4}} .
$$

Thus (4.111) and (4.116) yield that for $\varepsilon>0$ small enough and $i$ large enough (depending on $\varepsilon$ )

$$
\begin{aligned}
\left(1-C \varepsilon M^{n}\right) \int_{\partial \Omega_{i}} \varphi d \omega_{i}-C\|\varphi\|_{\infty} M^{n} \varepsilon^{\frac{1}{4}} & \leqslant \int_{\partial \Omega_{i}} \varphi d \sigma_{i} \\
& \leqslant\left(1+C M^{\frac{5 n}{4}} \varepsilon^{\frac{1}{4}}\right) \int_{\partial \Omega_{i}} \varphi d \omega_{i}+C M^{n}\|\varphi\|_{\infty} \varepsilon .
\end{aligned}
$$

Letting $i \rightarrow \infty$ in (4.117) and recalling (4.10) we have that for every $\varepsilon>0$

$$
\begin{gathered}
\left(1-C \varepsilon M^{n}\right) \int_{\partial \Omega_{\infty}} \varphi d \omega_{\infty}-C\|\varphi\|_{\infty} M^{n} \varepsilon^{\frac{1}{4}} \leqslant \liminf _{i \rightarrow \infty} \int_{\partial \Omega_{i}} \varphi d \sigma_{i}, \\
\limsup _{i \rightarrow \infty} \int_{\partial \Omega_{i}} \varphi d \sigma_{i} \leqslant\left(1+C M^{\frac{5 n}{4}} \varepsilon^{\frac{1}{4}}\right) \int_{\partial \Omega_{\infty}} \varphi d \omega_{\infty}+C M^{n}\|\varphi\|_{\infty} \varepsilon .
\end{gathered}
$$

Thus for every $\varphi \in C_{c}^{\infty}\left(\mathbb{R}^{n+1}\right), \varphi \geqslant 0$ we have

$$
\lim _{i \rightarrow \infty} \int_{\partial \Omega_{i}} \varphi d \sigma_{i}=\int_{\partial \Omega_{\infty}} \varphi d \omega_{\infty}
$$


Since $\Omega_{\infty}, u_{\infty}, \omega_{\infty}$ and $h_{\infty}$ satisfy the hypothesis of Theorem 2.2 we conclude that $d \omega_{\infty}=d \sigma_{\infty}$. Therefore $\sigma_{i} \rightarrow \sigma_{\infty}$ as $i \rightarrow \infty$ weakly as Radon measures.

We now recall the statement of the Main Theorem and present its proof.

MAIN THEOREM. - Assume that

(1) $\Omega \subset \mathbb{R}^{n+1}$ is a $\delta$-Reifenberg flat chord arc domain for some $\delta>0$ small enough.

(2) $\log h \in \operatorname{VMO}(d \sigma)$.

Then $\Omega$ is a chord arc domain with vanishing constant, i.e. $\vec{n} \in \operatorname{VMO}(d \sigma)$.

Proof. - Let $K \subset \mathbb{R}^{n+1}$ be a compact set, and let

$$
l=\lim _{r \rightarrow 0} \sup _{Q \in \partial \Omega \cap K}\|\vec{n}\|_{*}(B(Q, r)) .
$$

Our goal is to show that $l=0$. There exist sequences $\left\{Q_{i}\right\}_{i \geqslant 1} \subset \partial \Omega \cap K$, and $\left\{r_{i}\right\}_{i \geqslant 1} \subset \mathbb{R}$ such that $\lim _{i \rightarrow \infty} Q_{i}=Q_{\infty}, 0<r_{i}, \lim _{i \rightarrow \infty} r_{i}=0$ and

$$
\lim _{i \rightarrow \infty}\left(f_{B\left(Q_{i}, r_{i}\right)}\left|\vec{n}-\vec{n} Q_{i}, r_{i}\right|^{2} d \sigma\right)^{\frac{1}{2}}=l .
$$

We consider the blow up sequences $\Omega_{i}=r_{i}^{-1}\left(\Omega-Q_{i}\right), \partial \Omega_{i}=r_{i}^{-1}\left(\partial \Omega-Q_{i}\right), u_{i}, \omega_{i}$ and $h_{i}$ associated with $Q_{i}$ and $r_{i}$ as in (4.2), (4.4) and (4.5). Theorems 4.1, 4.2, and 4.3 combined with Theorem 2.2 ensure that by passing to a subsequence (which we relabel), and modulo rotation we have that

$$
\begin{aligned}
& \Omega_{i} \rightarrow \mathbb{R}_{+}^{n+1} \begin{array}{l}
\text { in the Hausdorff distance sense }, \\
\text { uniformly on compact sets, }
\end{array} \\
& \partial \Omega_{i} \rightarrow \mathbb{R}^{n} \times\{0\} \quad \begin{array}{l}
\text { in the Hausdorff distance sense }, \\
\text { uniformly on compact sets },
\end{array}
\end{aligned}
$$

and

$$
\sigma_{i}, \omega_{i} \rightarrow \mathcal{H}^{n}\left\llcorner\left(\mathbb{R}^{n} \times\{0\}\right) .\right.
$$

Recall also that $\chi_{\Omega_{i}} \rightarrow \chi_{\mathbb{R}_{+}^{n+1}}$ in $L_{\text {loc }}^{1}\left(\mathbb{R}^{n+1}\right)$, thus for $\varphi \in C_{c}^{\infty}\left(\mathbb{R}^{n+1}\right), \varphi \geqslant 0$ and $e \in \mathbb{S}^{n}$ we have

$$
\lim _{i \rightarrow \infty} \int_{\Omega_{i}} \operatorname{div}(\varphi e)=\int_{\mathbb{R}_{+}^{n+1}} \operatorname{div}(\varphi e) .
$$

If $\overrightarrow{n_{i}}$ denotes the inner unit normal to $\partial \Omega_{i}$ we have that

$$
\int_{\partial \Omega_{i}} \varphi\left\langle\overrightarrow{n_{i}}, e\right\rangle d \sigma_{i}=-\int_{\Omega_{i}} \operatorname{div}(\varphi e) .
$$

Therefore

$$
\lim _{i \rightarrow \infty} \int_{\partial \Omega_{i}} \varphi\left\langle\overrightarrow{n_{i}}, e\right\rangle d \sigma_{i}=\int_{\mathbb{R}^{n} \times\{0\}} \varphi\left\langle e_{n+1}, e\right\rangle d \mathcal{H}^{n},
$$


which can be rewritten as

$$
\begin{aligned}
\lim _{i \rightarrow \infty} \int_{\partial \Omega_{i}} \varphi d \sigma_{i}-\frac{1}{2} \int_{\partial \Omega_{i}} \varphi\left|\overrightarrow{n_{i}}-e\right|^{2} d \sigma_{i} \\
\quad=\int_{\mathbb{R}^{n} \times\{0\}} \varphi d \mathcal{H}^{n}-\frac{1}{2} \int_{\mathbb{R}^{n} \times\{0\}} \varphi\left|e_{n+1}-e\right|^{2} d \mathcal{H}^{n} .
\end{aligned}
$$

Theorem 4.4 yields

$$
\lim _{i \rightarrow \infty} \int_{\partial \Omega_{i}} \varphi\left|\overrightarrow{n_{i}}-e\right|^{2} d \sigma_{i}=\int_{\mathbb{R}^{n} \times\{0\}} \varphi\left|e_{n+1}-e\right|^{2} d \mathcal{H}^{n} .
$$

Letting $e=e_{n+1}$ and $\varphi \in C_{c}^{\infty}\left(\mathbb{R}^{n+1}\right), \varphi \geqslant \chi_{B(0,1)}$, (4.129) shows that

$$
\lim _{i \rightarrow \infty} \int_{B(0,1)}\left|\overrightarrow{n_{i}}-e_{n+1}\right|^{2} d \sigma_{i}=0 .
$$

Note that for $Q \in \partial \Omega_{i}, \overrightarrow{n_{i}}(Q)=\vec{n}\left(r_{i} Q+Q_{i}\right)$ where $\vec{n}$ denotes the inner unit normal to $\partial \Omega$. Furthermore

$$
\oint_{B(0,1)}\left|\overrightarrow{n_{i}}-e_{n+1}\right|^{2} d \sigma_{i}=\oint_{B\left(Q_{i}, r_{i}\right)}\left|\vec{n}-e_{n+1}\right|^{2} d \sigma .
$$

Combining (4.121), (4.130) and (4.131) we conclude that $l=0$. In fact note that

$$
\begin{aligned}
l & =\lim _{i \rightarrow \infty}\left(f_{B\left(Q_{i}, r_{i}\right)}\left|\vec{n}-\vec{n} Q_{i}, r_{i}\right|^{2} d \sigma\right)^{\frac{1}{2}} \\
& \leqslant 2 \lim _{i \rightarrow \infty}\left(f_{B\left(Q_{i}, r_{i}\right)}\left|\vec{n}-e_{n+1}\right|^{2} d \sigma\right)^{\frac{1}{2}}=0 .
\end{aligned}
$$

\section{Acknowledgements}

Part of this work was carried out while the authors were visiting MSRI, and also when the second author was visiting the Université de Paris Sud, Centre d'Orsay. We wish to thank MSRI and Orsay for their hospitality. We also wish to thank L. Caffarelli and G. David for very useful conversations. L. Caffarelli's advice was to blow up everything, G. David's to remove hypotheses and prove the same results. Their encouragement and enthusiasm were crucial to the successful completion of our work. We also would like to thank the referee for his valuable comments and suggestions.

\section{Appendix A}

The main purpose of this appendix is to prove Lemma 3.2 as well as Rellich's identity for chord-arc domains with small constant. We would like to thank G. David who pointed out to us that our proofs could be simplified, and that some of the results held in a more general class of domains.

This appendix is organized as follows: we first show that Reifenberg flat chord arc domains can be locally approximated from the interior by domains of a similar type. We use this 
approximation to show that if $\Omega$ is such a domain, and $F$ denotes the non-tangential limit of the gradient of Green's function with pole at infinity $u$ or of the gradient of the Green's function with pole at $A \in \Omega, G(A,-)$, then either $h(Q)=\langle F(Q), \vec{n}(Q)\rangle$ or $k_{A}(Q)=\langle F(Q), \vec{n}(Q)\rangle$ for $\mathcal{H}^{n}$ a.e. $Q \in \partial \Omega$. Here $h$ (resp. $k_{A}$ ) denote the Poisson kernel with pole at infinity (resp. the Poisson kernel with pole $A$ ). In the second part of the appendix we show that for $F$ as above, $F(Q)=h(Q) \vec{n}(Q)$ or $F(Q)=k_{A}(Q) \vec{n}(Q)$ for $\mathcal{H}^{n}$ a.e. $Q \in \partial \Omega$. The proof presented here is due to G. David. Our original proof made use of the parameterizations for chord-arc surfaces with small constant constructed by Semmes in [24]. In the third part of the appendix we prove Rellich's identity for chord-arc domains with small constant, verifying a point left open in [18].

\section{A.1. Approximation of Reifenberg flat chord-arc domains}

Recall that if $\Omega$ is a set of locally finite perimeter which is Reifenberg flat then the topological boundary of $\Omega$ and its measure theoretic boundary agree (see Remark 4.2 in [18]). Moreover $\mathcal{H}^{n}\left(\partial \Omega \backslash \partial^{*} \Omega\right)=0$; here $\partial^{*} \Omega$ denotes the reduced boundary of $\Omega$. This implies that for $\mathcal{H}^{n}\llcorner\partial \Omega$ a.e. $Q \in \partial \Omega$

$$
\lim _{r \rightarrow 0} \int_{B(Q, r) \cap \partial \Omega} \vec{n}(P) d \mathcal{H}^{n}(P)=\vec{n}(Q),
$$

$$
\begin{gathered}
|\vec{n}(Q)|=1, \\
\lim _{r \rightarrow 0} \frac{\mathcal{H}^{n}(\partial \Omega \cap B(Q, r))}{\omega_{n} r^{n}}=1 .
\end{gathered}
$$

See [7, Chapter 5]. Here $\vec{n}$ denote the inward unit normal vector to $\partial \Omega$.

We now begin the construction of the approximating domains. Let $\Omega \subset \mathbb{R}^{n+1}$ be a $\delta$ Reifenberg flat chord-arc domain. Fix $K_{0} \subset \mathbb{R}^{n+1}$ a compact set, and $R_{0}=R_{K_{0}}$ so that (1.8), (1.9), (1.10) hold. Let $Q_{0} \in K_{0} \cap \partial \Omega$, let $R \in\left(0, \frac{R_{0}}{4}\right)$, and let $\rho \in(0,1)$ be a small but fixed constant (to be determined later). Let $r_{j}=R \rho^{j}$, for $j \geqslant 1$. Let $\left\{P_{j i}\right\}_{i}$ be a finite subset of $\partial^{*} \Omega \cap B(Q, 2 R)$ satisfying

$$
\left|P_{j i}-P_{j l}\right| \geqslant r_{j} \quad \text { for } i \neq l
$$

and

$$
\partial \Omega \cap B\left(Q_{0}, 2 R\right) \subset \bigcup_{i} B\left(P_{j i}, r_{j}\right) \subset \bigcup_{i} B\left(P_{j i}, \frac{13 r_{j}}{4}\right) \subset B\left(Q_{0}, 6 R\right) .
$$

We denote by $\overrightarrow{n_{j i}}=\vec{n}\left(P_{j i}, r_{j}\right)$, where the notation is as in (1.9) and (1.10).

Let $\left\{\lambda_{j i}\right\}_{i}$ be a smooth partition of unity associated to $\left\{B\left(P_{j i}, r_{j}\right)\right\}$ satisfying

$$
\begin{cases}\lambda_{j i}(X)=1 & \text { if }\left|X-P_{j i}\right|<\frac{1}{4} r_{j} \\ \lambda_{j i}(X)=0 & \text { if }\left|X-P_{j i}\right| \geqslant \frac{13}{4} r_{j}\end{cases}
$$

and

$$
\text { (A.1.8) } \sum_{i} \lambda_{j i}(X)=0 \text { and } \sum_{i} \nabla \lambda_{j i}(X)=0 \quad \text { for } X \in\left(B\left(Q_{0}, 2 R\right) \cap \partial \Omega^{\prime} \frac{5 r_{j}}{2}\right) \text {. }
$$


Define for $X \in \mathbb{R}^{n+1}$ smooth functions $N_{j}$ and $\phi_{j}$ by

$$
N_{j}(X)=\sum_{i} \lambda_{j i}(X) \overrightarrow{n_{j i}}
$$

and

$$
\phi_{j}(X)=X+\alpha r_{j} N_{j}(X),
$$

where $\alpha$ denotes a small positive constant much larger than $\sqrt{\delta}$. $\alpha$ will be determined later as a function of $\delta$. Note that if $X \notin \bigcup_{i} B\left(P_{j i}, \frac{13 r_{j}}{4}\right)$ then $N_{j}(X)=0$ and $\phi_{j}(X)=X$. Our goal is to show that $\phi_{j}$ is a bilipschitz map from $\mathbb{R}^{n+1}$ to $\mathbb{R}^{n+1}$, with constants close to 1 (depending on $\alpha>0$ ). To do this we need to estimate $N_{j}(X)-N_{j}(Y)$. Since $N_{j}(Z)=0$ for $Z \notin \bigcup_{i} B\left(P_{j i}, \frac{13 r_{j}}{4}\right)$, we only need to consider 2 cases. Either $X \in \bigcup_{i} B\left(P_{j i}, \frac{13 r_{j}}{4}\right)$ and $Y \notin \bigcup_{i} B\left(P_{j i}, \frac{13 r_{j}}{4}\right)$ or both $X, Y \in \bigcup_{i} B\left(P_{j i}, \frac{13 r_{j}}{4}\right)$. Since $\left\{B\left(P_{j i}, \frac{13 r_{j}}{4}\right)\right\}_{i}$ is a disjoint collection, then $X$ only belongs to at most $K_{n}$ balls in the collection $\left\{B\left(P_{j i}, \frac{r_{j}}{4}\right)\right\}$, where $K_{n}$ is a constant independent of $r_{j}$ (only depending on $n$ ). If $Y \notin \bigcup_{i} B\left(P_{j i}, \frac{13 r_{j}}{4}\right)$ there is $Z \in \partial B\left(P_{j i_{o}}, \frac{B r_{j}}{4}\right)$ for some $i_{0}$ such that

$$
|X-Z| \leqslant|Y-X|
$$

since $N_{j}(Y)=N_{j}(Z)=0$ then

$$
\begin{aligned}
N_{j}(X)-N_{j}(Y) & =N_{j}(X)-N_{j}(Z)=\sum_{i}\left(\lambda_{j i}(X)-\lambda_{j i}(Z)\right) \overrightarrow{n_{j i}} \\
& =\sum_{\left|X-P_{j i}\right| \leqslant \frac{13 r_{j}}{4}}\left(\lambda_{j i}(X)-\lambda_{j i}(Z)\right) \overrightarrow{n_{j i}} .
\end{aligned}
$$

Since $\left|\overrightarrow{n_{j i}}\right|=1$ then

$$
\left|N_{j}(X)-N_{j}(Y)\right| \leqslant \frac{C_{n}}{r_{j}}|X-Y|
$$

and

$$
\left(1-C_{n} \alpha\right)|X-Y| \leqslant\left|\phi_{j}(X)-\phi_{j}(Y)\right| \leqslant\left(1+C_{n} \alpha\right)|X-Y| .
$$

Now we need to analyze the case when $Y \in \bigcup_{i} B\left(P_{j i}, \frac{13 r_{j}}{4}\right)$. If $X \in B\left(P_{j i}, \frac{13 r_{j}}{4}\right)$ and $Y \notin B\left(P_{j i}, \frac{13 r_{j}}{4}\right)$, choose $X_{j i} \in \partial B\left(P_{j i}, \frac{13 r_{j}}{4}\right)$ so that $\left|X-X_{j i}\right| \leqslant|X-Y|$. Similarly if $Y \in B\left(P_{j l}, \frac{13 r_{j}}{4}\right)$ and $X \notin B\left(P_{j l}, \frac{13 r_{j}}{4}\right)$ choose $Y_{j l} \in \partial B\left(P_{j l}, \frac{13 r_{j}}{4}\right)$ so that

$$
\left|Y-Y_{j l}\right| \leqslant|Y-X|
$$

Using this notation we have that

$$
\begin{aligned}
N_{j}(X)-N_{j}(Y)= & \sum_{i}\left(\lambda_{j i}(X)-\lambda_{j i}(Y)\right) \overrightarrow{n_{j i}} \\
= & \sum_{\left|X-P_{j i}\right| \leqslant \frac{13 r_{j}}{4},\left|Y-P_{j i}\right| \leqslant \frac{13 r_{j}}{4}}\left(\lambda_{j i}(X)-\lambda_{j i}(Y)\right) \overrightarrow{n_{j i}} \\
& +\sum_{\left|X-P_{j i}\right| \leqslant \frac{13 r_{j}}{4},\left|Y-P_{j i}\right|>\frac{13 r_{j}}{4}}\left(\lambda_{j i}(X)-\lambda_{j i}\left(X_{j i}\right)\right) \overrightarrow{n_{j i}}
\end{aligned}
$$




$$
+\sum_{\left|X-P_{j i}\right|>\frac{13 r_{j}}{4},\left|Y-P_{j i}\right| \leqslant \frac{13 r_{j}}{4}}\left(\lambda_{j i}\left(Y_{j l}\right)-\lambda_{j i}(Y)\right) \overrightarrow{n_{j i}} .
$$

Thus using the finite intersection property of the coverings involved, and the choice of $X_{j i}$ and $Y_{j l}$ we have

$$
\begin{aligned}
\left|N_{j}(X)-N_{j}(Y)\right| \leqslant & \sum_{\left|X-P_{j i}\right| \leqslant \frac{13 r_{j}}{4},\left|Y-P_{j i}\right| \leqslant \frac{13 r_{j}}{4}} \frac{C_{n}}{r_{j}}|X-Y| \\
& +\sum_{\left|x-P_{j i}\right| \leqslant \frac{13 r_{j}}{4},\left|Y-P_{j i}\right|>\frac{13 r_{j}}{4}} \frac{C_{n}}{r_{j}}\left|X-X_{j i}\right| \\
& +\sum_{\left|X-P_{j i}\right|>\frac{13 r_{j}}{4},\left|Y-P_{j i}\right| \leqslant \frac{13 r_{j}}{4}} \frac{C_{n}}{r_{j}}\left|Y_{j l}-Y\right| \\
\leqslant & \frac{C_{n}}{r_{j}}|X-Y|,
\end{aligned}
$$

which once again implies that

$$
\left(1-C_{n} \alpha\right)|X-Y| \leqslant\left|\phi_{j}(X)-\phi_{j}(Y)\right| \leqslant\left(1+C_{n} \alpha\right)|X-Y| .
$$

Thus $\phi_{j}$ is a bilipschitz map from $\mathbb{R}^{n+1}$ into $\mathbb{R}^{n+1}$ with constants $\left(1+C_{n} \alpha\right)$ for $\alpha$ small enough. In particular $\phi_{j}$ is a homeomorphism from $\mathbb{R}^{n+1}$ onto its image which coincides with the identity outside the ball $B\left(Q_{0}, 6 R\right)$. A simple argument shows that $\phi_{j}\left(\mathbb{R}^{n+1}\right)=\mathbb{R}^{n+1}$.

Define

$$
\Omega_{j}=\phi_{j}(\Omega) .
$$

Since $\phi$ is a homeomorphism from $\mathbb{R}^{n+1}$ onto $\mathbb{R}^{n+1}$

$$
\partial \Omega_{j}=\phi_{j}(\partial \Omega)
$$

LEMMA A.1.1. - There exist $\alpha_{n}, \delta_{n}>0$ so that if $\Omega$ is a $\delta$-Reifenberg flat chord arc domain (for $\delta<\delta_{n}$ ) and $\Omega_{j}=\phi_{j}(\Omega)$, with $\phi_{j}$ defined as above with $\alpha<\alpha_{n}$ then for each $j \geqslant 1, \Omega_{j}$ is a chord arc domain. Moreover

$$
\Omega_{j} \rightarrow \Omega \text { in the Hausdorff distance sense }
$$

and

$$
\partial \Omega_{j} \rightarrow \partial \Omega \text { in the Hausdorff distance sense. }
$$

Proof. - Our initial goal is to show that bilipschitz maps transform sets of locally finite perimeter into sets of locally finite perimeter. Due to the lack of a reference we present the proof here. Note that given $\varphi \in C_{c}^{1}\left(\mathbb{R}^{n+1}, \mathbb{R}^{n+1}\right)$ with $|\varphi| \leqslant 1$

$$
\begin{aligned}
\int_{\Omega_{j}} \operatorname{div} \varphi(Y) d Y & =\int_{\Omega} \operatorname{div} \varphi\left(\phi_{j}(Y)\right) J \phi_{j}(Y) d Y \\
& =\int_{\Omega} \operatorname{div} \varphi\left(\phi_{j}(Y)\right) d Y+\int_{\Omega} \operatorname{div} \varphi\left(\phi_{j}(Y)\right)\left(J \phi_{j}(Y)-1\right) d Y .
\end{aligned}
$$


Since $\Omega$ is a $\delta$-Reifenberg flat chord arc domain for $\varphi \in C_{c}^{1}\left(\mathbb{R}^{n+1}, \mathbb{R}^{n+1}\right),|\varphi| \leqslant 1$ then

$$
\int_{\Omega} \operatorname{div} \varphi\left(\phi_{j}(Y)\right) d Y=\int_{\partial \Omega} \varphi\left(\phi_{j}(Y)\right) \cdot \vec{n}(Y) d \mathcal{H}^{n}(Y),
$$

and if $\operatorname{spt} \varphi \subset B\left(Q, R_{1}\right)$ with $R_{1} \geqslant 6 R$

$$
\left|\int_{\Omega} \operatorname{div} \varphi\left(\phi_{j}(Y)\right) d Y\right| \leqslant \mathcal{H}^{n}\left(\partial \Omega \cap B\left(Q, R_{1}\right)\right)<\infty
$$

On the other hand since $\phi_{j}=i d$ outside $B\left(Q_{0}, 6 R\right)$ and smooth in $\mathbb{R}^{n+1}$

$$
\begin{aligned}
\left|\int_{\Omega} \operatorname{div}\left(\varphi\left(\phi_{j}(Y)\right)\right)\left(J \phi_{j}(Y)-1\right) d Y\right| & \left|\int_{\Omega \cap B\left(Q, R_{1}\right)} \operatorname{div}\left(\varphi\left(\phi_{j}(Y)\right)\right)\left(J \phi_{j}(Y)-1\right) d Y\right| \\
= & \left|\int_{\Omega \cap B\left(Q, R_{1}\right)}\left[\operatorname{div}\left(\varphi\left(\phi_{j}(Y)\right)\left(J \phi_{j}(Y)-1\right)\right)-\varphi\left(\phi_{j}(Y)\right) \nabla J \phi_{j}(Y)\right] d Y\right| \\
\leqslant & \left|\int_{\Omega \cap B\left(Q, R_{1}\right)} \operatorname{div}\left(\varphi\left(\phi_{j}(Y)\right)\left(J \phi_{j}(Y)-1\right)\right) d Y\right| \\
& +\left|\int_{\Omega \cap B\left(Q, R_{1}\right)} \varphi\left(\phi_{j}(Y)\right) \nabla J \phi_{j}(Y) d Y\right| \\
\leqslant & \left|\int_{\partial \Omega \cap B\left(Q, R_{1}\right)} \varphi\left(\phi_{j}(Y)\right)\left(J\left(\phi_{j}(Y)\right)-1\right) \cdot \vec{n}(Y) d \mathcal{H}^{n}(Y)\right| \\
& +\int_{\Omega \cap B\left(Q, R_{1}\right)}\left|D^{2} \phi_{j}(Y)\right| d Y
\end{aligned}
$$

(A.1.25) $\leqslant C_{n} \mathcal{H}^{n}\left(\partial \Omega \cap B\left(Q, R_{1}\right)\right)+\int_{\Omega \cap B\left(Q, R_{1}\right)}\left|D^{2} \phi_{j}(Y)\right| d Y$.

Note that by definition (A.1.10)

$$
D^{2} \phi_{j}(Y)=\alpha r_{j} D^{2} N_{j}(Y)=\alpha r_{j} \sum_{i} \nabla^{2} \lambda_{j i}(X) \overrightarrow{n_{j i}}
$$

Thus

$$
\text { (A.1.27) } \quad\left|D^{2} \phi_{j}(Y)\right| \leqslant \alpha r_{j} \sum_{\left|X-P_{j i}\right| \leqslant \frac{13 r_{j}}{4}}\left|\nabla^{2} \lambda_{j i}(X)\right| \leqslant \alpha r_{j} \frac{C_{n}}{r_{j}^{2}}=\frac{C_{n}}{r_{j}} \alpha,
$$

and (A.1.25) becomes 


$$
\begin{aligned}
& \left|\int_{\Omega} \operatorname{div}\left(\varphi\left(\phi_{j}(Y)\right)\right)(J \phi(Y)-1) d Y\right| \\
& \quad \leqslant C_{n} \mathcal{H}^{n}\left(\partial \Omega \cap B\left(Q, R_{1}\right)\right)+\frac{\alpha C_{n}}{r_{j}} R_{1}^{n+1} .
\end{aligned}
$$

Combining (A.1.22), (A.1.23), (A.1.24) and (A.1.28) we conclude that for each $j \geqslant 1, \Omega_{j}$ is a set of locally finite perimeter. Since $\partial \Omega$ is Ahlfors regular there exists $C>1$ so that for $Q \in \partial \Omega$, and $r>0$

$$
C^{-1} r^{n} \leqslant \mathcal{H}^{n}(\partial \Omega \cap B(Q, r)) \leqslant C r^{n} .
$$

Since $\partial \Omega_{j}=\phi_{j}(\Omega)$ and $\operatorname{Lip} \phi_{j}, \operatorname{Lip} \phi_{j}^{-1} \leqslant 1+C_{n} \alpha$ then for $P_{j} \in \partial \Omega_{j} \cap K$ and $r \in\left(0, R_{1}\right)$ if $P_{j}=\phi_{j}(P)$ with $P \in \partial \Omega \cap \phi_{j}^{-1}(K)$ then provided that $C_{n} \alpha<1$ we have

$$
\begin{aligned}
\phi_{j}\left(\partial \Omega \cap B\left(P, \frac{r}{1+C_{n} \alpha}\right)\right) & \subset \partial \Omega_{j} \cap B\left(P_{j}, r\right) \\
& \subset \phi_{j}\left(\partial \Omega \cap B\left(P,\left(1+C_{n} \alpha\right) r\right)\right),
\end{aligned}
$$

which implies that for $\alpha$ small enough

$$
\begin{aligned}
\mathcal{H}^{n}\left(\partial \Omega_{j} \cap B\left(P_{j}, r\right)\right) & \leqslant \mathcal{H}^{n}\left(\phi_{j}\left(\partial \Omega \cap B\left(P,\left(1+C_{n} \alpha\right) r\right)\right)\right) \\
& \leqslant\left(\operatorname{Lip} \phi_{j}\right)^{n} \mathcal{H}^{n}\left(\partial \Omega \cap B\left(P,\left(1+C_{n} \alpha\right) r\right)\right) \\
& \leqslant C_{0} r^{n},
\end{aligned}
$$

(see [7, Section 2.4] for a justification of the second inequality). Similarly

$$
\begin{aligned}
\mathcal{H}^{n}\left(\partial \Omega \cap B\left(P, \frac{r}{1+C_{n} \alpha}\right)\right) & \leqslant \mathcal{H}^{n}\left(\phi_{j}^{-1}\left(\partial \Omega_{j} \cap B\left(P_{j}, r\right)\right)\right) \\
& \leqslant\left(\operatorname{Lip} \phi_{j}^{-1}\right)^{n} \mathcal{H}^{n}\left(\partial \Omega_{j} \cap B\left(P_{j}, r\right)\right),
\end{aligned}
$$

and

$$
C_{0}^{-1} r^{n} \leqslant \mathcal{H}^{n}\left(\partial \Omega_{j} \cap B\left(P_{j}, r\right)\right) .
$$

We have that for each $j \geqslant 1, \Omega_{j}$ is a set of locally finite perimeter whose boundary $\partial \Omega_{j}$ is Ahlfors regular. To show that $\Omega_{j}$ is a chord arc domain we need to prove that $\Omega_{j}$ is an NTA domain. To do this we note that the image of an NTA domain via a bilipschitz map is an NTA domain. Since $\Omega$ is NTA there exist $M>1$ and $R>0$ so that

(A.1.34) Corkscrew condition. For any $P \in \partial \Omega, r<R$ there exists $A=A(r, P) \in \Omega$ such that $M^{-1} r<|A-P|<r$ and $d(A, \partial \Omega)>M^{-1} r$.

(A.1.35) $\Omega^{c}$ satisfies the corkscrew condition.

(A.1.36) Harnack Chain Condition. If $\varepsilon>0$, and $X_{1}, X_{2} \in \Omega \cap B\left(P, \frac{r}{4}\right)$ for some $P \in \partial \Omega$, $r<R, d\left(X_{j}, \partial \Omega\right)>\varepsilon$ and $\left|X_{1}-X_{2}\right|<2^{k} \varepsilon$, then there exists a Harnack chain from $X_{1}$ to $X_{2}$ of length $M k$ and such that the diameter of each ball is bounded below by $M^{-1} \min \left\{\operatorname{dist}\left(X_{1}, \partial \Omega\right)\right.$, $\left.\operatorname{dist}\left(X_{2}, \partial \Omega\right)\right\}$. 
Let $\bar{R}=\left(1+C_{n} \alpha\right)^{-1} R$, let $P_{j} \in \partial \Omega_{j}$, and $r<\bar{R}$. Since $P_{j}=\phi_{j}(P)$ for some $P \in \partial \Omega$, then there exists $A=A(P, r) \in \Omega$ such that

$$
\text { (A.1.37) } M^{-1} \frac{r}{1+C_{n} \alpha}<|A-P|<\frac{r}{1+C_{n} \alpha} \quad \text { and } \quad \operatorname{dist}(A, \partial \Omega)>M^{-1} \frac{r}{1+C_{n} \alpha}
$$

therefore $A_{j}=\phi_{j}(A) \in \Omega_{j}$ and

$$
\begin{gathered}
M^{-1}\left(1+C_{n} \alpha\right)^{-2} r<\left|\phi_{j}(A)-P_{j}\right|<r \quad \text { and } \\
\operatorname{dist}\left(A_{j}, \partial \Omega_{j}\right)>M^{-1} \frac{r}{\left(1+C_{n} \alpha\right)^{-2}} .
\end{gathered}
$$

Thus $\Omega_{j}$ satisfies the corkscrew condition with constant $\bar{M}=M\left(1+C_{n} \alpha\right)^{2}$ and for $r<\bar{R}$. Similarly $\Omega^{c}$ satisfies the corkscrew condition with the same constants. In order to verify that the Harnack chain holds let $\varepsilon>0$ and $X_{1}^{j}, X_{2}^{j} \in \Omega_{j} \cap B\left(P_{j}, \frac{r}{4}\right)$ for some $P_{j} \in \partial \Omega_{j}$, $\operatorname{dist}\left(X_{i}^{j}, \partial \Omega_{j}\right)>\varepsilon$ for $i=1,2$ and $\left|X_{1}^{j}-X_{2}^{j}\right|<2^{k} \varepsilon$. If $P_{j}=\phi_{j}(P)$ and $X_{i}^{j}=\phi\left(X_{i}\right)$ then

$$
P \in \partial \Omega \quad X_{1}, X_{2} \in \Omega \cap B\left(P, \frac{r\left(1+C_{n} \alpha\right)}{4}\right), \quad \operatorname{dist}\left(X_{i}, \partial \Omega\right)>\varepsilon\left(1+C_{n} \alpha\right)
$$

and $\left|X_{1}-X_{2}\right|<2^{k} \varepsilon\left(1+C_{n} \alpha\right)$. Since $\Omega$ in NTA, there exists a Harnack chain $\left\{B\left(Y_{l}, r_{l}\right)\right\}_{l=1}^{M k}$ joining $X_{1}$ to $X_{2}$ satisfying the condition above. Using the fact that $\phi_{j}$ is bilipschitz and $\operatorname{Lip} \phi_{j} \leqslant 1+C_{n} \alpha$, it is not difficult to check that the collection $\left\{B\left(\phi_{j}\left(Y_{l}\right),\left(1+C_{n} \alpha\right) r_{l}\right)\right\}_{l=1}^{M k}$ forms a Harnack chain joining $\phi_{j}\left(X_{1}\right)$ to $\phi_{j}\left(X_{2}\right)$ and satisfying the diameter condition above. Therefore $\Omega_{j}$ is an NTA domain and hence a chord arc domain. To conclude the proof of Lemma A.1.1 we need to show that the $\Omega_{j}$ 's (resp. $\partial \Omega_{j}$ 's) converge to $\Omega$ (resp. $\Omega$ ) in the Hausdorff distance sense. Since $\phi_{j}=i d$ on $\mathbb{R}^{n+1} \backslash B(Q, 6 R)$ by (A.1.4), (A.1.9) and (A.1.10) then $\Omega=\Omega_{j}, \Omega^{c}=\Omega_{j}^{c}$ and $\partial \Omega=\partial \Omega_{j}$ on $\mathbb{R}^{n+1} \backslash B(Q, 6 R)$. For $X \in B(Q, 6 R),(\mathrm{A} .1 .7)$ and the finite intersection property of the collection $\left\{B\left(P_{j i}, \frac{13 r_{j}}{4}\right)\right\}$ ensure that

$$
\left|N_{j}(X)\right| \leqslant \sum_{i} \lambda_{j i}(X)\left|\overrightarrow{n_{j i}}\right| \leqslant \sum_{\left|X-P_{j i}\right| \leqslant \frac{13 r_{j}}{4}} \lambda_{j i}(X) \leqslant K_{n}
$$

Therefore

$$
\left|\phi_{j}(X)-X\right| \leqslant \alpha K_{n} r_{j}
$$

Since $\phi_{j}(\Omega)=\Omega_{j}, \phi_{j}\left(\Omega^{c}\right)=\Omega_{j}^{c}$ and $\phi_{j}(\partial \Omega)=\partial \Omega_{j}$ we have that

$$
\Omega^{c} \subset\left(\Omega_{j}^{c}, \alpha K_{n} r_{j}\right) \text { and } \partial \Omega \subset\left(\partial \Omega_{j}, \alpha K_{n} r_{j}\right) .
$$

Since $\phi_{j}$ is a homeomorphism from $\mathbb{R}^{n+1}$ onto $\mathbb{R}^{n+1}$, for each $Y_{j} \in \Omega_{j}^{c}$ (resp. $P_{j} \in \partial \Omega_{j}$ ) there exists $Y \in \Omega^{c}$ (resp. $P \in \partial \Omega$ ) so that $\phi_{j}(Y)=Y_{j}$ (resp. $\phi_{j}(P)=P_{j}$ ). Hence (A.1.40) implies that

$$
\Omega_{j}^{c} \subset\left(\Omega^{c}, 2 \alpha K_{n} r_{j}\right) \text { and } \partial \Omega_{j} \subset\left(\partial \Omega, \alpha K_{n} r_{j}\right) .
$$

Combining (A.1.41) and (A.1.42) we have that

$$
D\left[\Omega_{j}^{c}, \Omega^{c}\right] \leqslant \alpha K_{n} r_{j} \quad \text { and } \quad D\left[\partial \Omega_{j}, \partial \Omega\right] \leqslant \alpha K_{n} r_{j} .
$$

Since $r_{j} \rightarrow 0$ as $j \rightarrow 0$ this concludes the proof of Lemma A.1.1. 
We now study the local properties of $\overline{\Omega_{j}}$ near $Q_{0}$, where $Q_{0} \in \partial \Omega \cap K_{0}$ is as in (A.1.4).

LEMMA A.1.2. - There exist $\alpha_{n}, \delta_{n}>0$ so that if $\Omega$ is a $\delta$-Reifenberg flat chord arc domain (for $\left.\delta<\delta_{n}\right)$ and $\Omega_{j}=\phi_{j}(\Omega)$ for $j \geqslant 1$ with $\phi_{j}$ defined as above with $\alpha<\alpha_{n}$ then

$$
\bar{\Omega}_{j} \cap B\left(Q_{0}, \frac{3 R}{2}\right) \subset \Omega \cap B\left(Q_{0}, \frac{3 R}{2}\right)
$$

and

$$
\mathcal{H}^{n}\left\llcorner\partial \Omega_{j} \underset{j \rightarrow \infty}{\rightarrow} \mathcal{H}^{n}\llcorner\partial \Omega\right.
$$

weakly as Radon measures. Moreover if $\overrightarrow{n_{j}}$ denotes the inward unit normal to $\partial \Omega_{j}$ then for $P \in \partial^{*} \Omega$

$$
\overrightarrow{n_{j}}\left(\phi_{j}(P)\right) \underset{j \rightarrow \infty}{\longrightarrow} \vec{n}(P) .
$$

Proof. - Let $X_{j} \in \bar{\Omega}_{j} \cap B\left(Q_{0}, \frac{3 R}{2}\right)$, there exists $X \in \bar{\Omega}$ so that

$$
\phi_{j}(X)=X_{j}
$$

If $X \notin \bigcup_{i} B\left(P_{j i}, \frac{13 r_{j}}{4}\right)$ then

$$
\phi_{j}(X)=X=X_{j} \in \Omega \cap B\left(Q_{0}, \frac{3 R}{2}\right)
$$

Thus we are only concerned with the case when $X \in \bigcup_{i} B\left(P_{j i}, \frac{13 r_{j}}{4}\right)$. Let $X \in B\left(P_{j i}, \frac{13 r_{j}}{4}\right)$, and let $\overrightarrow{\nu_{j i}}=\vec{n}\left(P_{j i}, \frac{13}{4} r_{j}\right)$. Then either $\left\langle X-P_{j i}, \overrightarrow{\nu_{j i}}\right\rangle \geqslant \sqrt{\alpha} r_{j}$ or $\left\langle X-P_{j i}, \overrightarrow{\nu_{j i}}\right\rangle<\sqrt{\alpha} r_{j}$. Before looking at each case separately we need to estimate the angle $\theta$ between $\overrightarrow{\nu_{j i}}$ and $\overrightarrow{n_{j i}}$.

Using (1.8) we know that

$$
\begin{aligned}
D & {\left[L\left(P_{j i}, \frac{13}{4} r_{j}\right) \cap B\left(P_{j i}, r_{j}\right), L\left(P_{j i}, r_{j}\right) \cap B\left(P_{j i}, r_{j}\right)\right] } \\
\leqslant & D\left[L\left(P_{j i}, \frac{13}{4} r_{j}\right) \cap B\left(P_{j i}, r_{j}\right), \partial \Omega \cap B\left(P_{j i}, r_{j}\right)\right] \\
& \quad+D\left[\partial \Omega \cap B\left(P_{j i}, r_{j}\right), L\left(P_{j i}, r_{j}\right) \cap B\left(P_{j i}, r_{j}\right)\right] \\
\leqslant & D\left[L\left(P_{j i}, \frac{13}{4} r_{j}\right) \cap B\left(P_{j i}, \frac{13}{4} r_{j}\right), \partial \Omega \cap B\left(P_{j i}, \frac{13 r_{j}}{4}\right)\right]+2 \delta r_{j}
\end{aligned}
$$

(A.1.47) $\leqslant \frac{13}{2} \delta r_{j}+2 \delta r_{j} \leqslant 9 \delta r_{j}$.

Therefore

$$
\cos \theta \geqslant 1-C \delta^{2} \text {. }
$$

In order to show that if $X \in B\left(P_{j i}, \frac{13 r_{j}}{4}\right) \cap \bar{\Omega}$ then $\phi_{j}(X) \in \Omega$ first consider the case when $\left\langle X-P_{j i}, \overrightarrow{\nu_{j i}}\right\rangle \geqslant \sqrt{\alpha} r_{j}$. Since $\left|N_{j}(X)\right| \leqslant \sum_{i} \lambda_{j i}(X)\left|\overrightarrow{n_{j i}}\right| \leqslant K_{n}$, the fact that

$$
\left\langle X-P_{j i}, \overrightarrow{\nu_{j i}}\right\rangle \geqslant \sqrt{\alpha} r_{j}
$$

guarantees that

$4^{\mathrm{e}}$ SÉRIE - TOME $36-2003-\mathrm{N}^{\circ} 3$ 
(A.1.49)

$$
\begin{aligned}
\left\langle\phi_{j}(X)-P_{j i}, \overrightarrow{\nu_{j i}}\right\rangle & =\left\langle X-P_{j i}, \overrightarrow{\nu_{j i}}\right\rangle+\left\langle\alpha r_{j} N_{j}(X), \overrightarrow{\nu_{j i}}\right\rangle \\
& \geqslant \sqrt{\alpha} r_{j}+\alpha r_{j}\left\langle N_{j}(X), \overrightarrow{\nu_{j i}}\right\rangle \\
& \geqslant \sqrt{\alpha} r_{j}-K_{n} \alpha r_{j} \\
& \geqslant \sqrt{\alpha} r_{j}\left(1-K_{n} \sqrt{\alpha}\right) .
\end{aligned}
$$

Provided that $\alpha$ is small enough so that $K_{n} \sqrt{\alpha}<\frac{1}{2}$, and that $\delta$ is small enough depending on $\alpha$ so that $\frac{1}{2} \sqrt{\alpha}>\frac{13}{2} \delta$ we conclude that $\left\langle X-P_{j i}, \overrightarrow{\nu_{j i}}\right\rangle \geqslant 2 \delta \frac{13 r_{j}}{4}$, which by (1.9) implies that $\phi_{j}(X) \in \Omega$ (by our choice of $R$ and $\rho>0$ ).

Now we consider the case when $\left\langle X-P_{j i}, \overrightarrow{\nu_{j i}}\right\rangle<\sqrt{\alpha} r_{j}$ since $X \in \bar{\Omega}$, (1.9) implies that

$$
-\frac{13 r_{j}}{2} \delta \leqslant\left\langle X-P_{j i}, \overrightarrow{\nu_{j i}}\right\rangle<\sqrt{\alpha} r_{j}
$$

If $L\left(P_{j i}, \frac{13 r_{j}}{4}\right)$ denotes the plane through $P_{j i}$ orthogonal to $\overrightarrow{\nu_{j i}}$, (A.1.50) implies that

$$
\operatorname{dist}\left(X, L\left(P_{j i}, \frac{13 r_{j}}{4}\right) \cap B\left(P_{j i}, \frac{13 r_{j}}{4}\right)\right)<\sqrt{\alpha} r_{j},
$$

and (1.9) guarantees that

$$
\operatorname{dist}\left(X, \partial \Omega \cap B\left(P_{j i}, \frac{13 r_{j}}{4}\right)\right)<\sqrt{\alpha} r_{j}+\frac{13}{2} \delta r_{j} .
$$

Hence there exists $Q \in \partial \Omega \cap B\left(P_{j i}, \frac{13 r_{j}}{4}\right)$ so that

$$
|X-Q|<\sqrt{\alpha} r_{j}+\frac{13}{2} \delta r_{j}<2 \sqrt{\alpha} r_{j}
$$

whenever $\frac{13}{2} \delta<\sqrt{\alpha}$. Using (A.1.14)

(A.1.54)

$$
\begin{aligned}
\left|Q-Q_{0}\right| & \leqslant|Q-X|+\left|X-Q_{0}\right| \leqslant\left|X-Q_{0}\right|+2 \sqrt{\alpha} r_{j} \\
& <\left(1+C_{n} \alpha\right)\left|\phi_{j}^{-1}(X)-\phi_{j}^{-1}\left(Q_{0}\right)\right|+2 \sqrt{\alpha} r_{j} \\
& <\left(1+C_{n} \alpha\right)\left(\left|X_{j}-Q_{0}\right|+\left|Q_{0}-\phi_{j}^{-1}\left(Q_{0}\right)\right|\right)+2 \sqrt{\alpha} r_{j} \\
& <\left(1+C_{n} \alpha\right) \frac{3 R}{2}+\left(1+C_{n} \alpha\right)^{2}\left|\phi_{j}\left(Q_{0}\right)-Q_{0}\right|+2 \sqrt{\alpha} R \\
& \leqslant\left(1+C_{n} \alpha\right) \frac{3}{2} R+\left(1+C_{n} \alpha\right)^{2} \alpha r_{j}\left|N_{j}\left(Q_{0}\right)\right|+2 \sqrt{\alpha} R \\
& \leqslant\left(\frac{3}{2}+2 \sqrt{\alpha}+C_{n} \alpha\right) R .
\end{aligned}
$$

Choosing $\alpha$ and $\delta>0$ so that $2 \sqrt{\alpha}+C_{n} \alpha<\frac{1}{8}$ and $\frac{13}{2} \delta<\sqrt{\alpha}$ we have that

$$
\left|Q-Q_{0}\right|<2 R
$$

Thus by (A.1.53) and (A.1.55)

$$
\operatorname{dist}\left(X, \partial \Omega \cap B\left(Q_{0}, 2 R\right)\right)<2 \sqrt{\alpha} r_{j} .
$$


Moreover there exists $P_{j l}$ so that $\left|Q-P_{j l}\right|<r_{j}$ and $\left|X-P_{j l}\right|<r_{j}(1+2 \sqrt{\alpha})$. Our goal now is to show that if $\overrightarrow{\nu_{j l}}=\vec{n}\left(P_{j l},(1+2 \sqrt{\alpha}) r_{j}\right)$ then $\left\langle\phi_{j}(X)-P_{j l}, \overrightarrow{\nu_{j l}}\right\rangle>2 \delta\left(1+2 \sqrt{\alpha} r_{j}\right)$, which by (1.9) implies that $\phi_{j}(X) \in \Omega$. Since

$$
\left\langle\phi_{j}(X)-P_{j l} \overrightarrow{\nu_{j l}}\right\rangle=\left\langle X-P_{j l}, \overrightarrow{\nu_{j l}}\right\rangle+\alpha r_{j}\left\langle N_{j}(X), \overrightarrow{\nu_{j l}}\right\rangle
$$

and since $X \in \bar{\Omega}$, by (1.9) $\left\langle X-P_{j l}, \overrightarrow{\nu_{j l}}\right\rangle \geqslant-2 \delta(1+2 \sqrt{\alpha}) r_{j}$. Then (A.1.57) becomes

$$
\begin{aligned}
\left\langle\phi_{j}(X)-P_{j l}, \overrightarrow{\nu_{j l}}\right\rangle \geqslant & -6 \delta r_{j}+\alpha r_{j}\left\langle N_{j}(Q), \overrightarrow{\nu_{j l}}\right\rangle \\
& +\alpha r_{j}\left\langle N_{j}(X)-N_{j}(Q), \overrightarrow{\nu_{j l}}\right\rangle .
\end{aligned}
$$

Using (A.1.13) and (A.1.53) we have that

$$
\left|\left\langle N_{j}(X)-N_{j}(Q), \overrightarrow{\nu_{j l}}\right\rangle\right| \leqslant C_{n} \sqrt{\alpha} .
$$

Recall that $N_{j}(Q)=\sum_{k} \lambda_{j k}(Q) \overrightarrow{n_{j k}}$ and

$$
\left\langle N_{j}(Q), \overrightarrow{\nu_{j l}}\right\rangle=\sum_{k} \lambda_{j k}(Q)\left\langle\overrightarrow{n_{j k}}, \overrightarrow{\nu_{j l}}\right\rangle
$$

A similar argument to the one used to show (A.1.48) with $\frac{13}{4}$ replaced by $1+2 \sqrt{\alpha}$ shows that

$$
\left\langle\overrightarrow{n_{j k}}, \overrightarrow{\nu_{j l}}\right\rangle \geqslant 1-C \delta^{2}
$$

Combining (A.1.60) and (A.1.61) we have that since $Q \in \partial \Omega \cap B\left(Q_{0}, 2 R\right)$ by (A.1.8)

$$
\left\langle N_{j}(Q), \overrightarrow{\nu_{j l}}\right\rangle \geqslant \sum_{k} \lambda_{j k}(Q)(1-\delta)\left(1-C_{n} \delta^{2}\right) \geqslant 1-2 \delta
$$

From (A.1.58), (A.1.59) and (A.1.62) we deduce that

$$
\begin{aligned}
\left\langle\phi_{j}(X)-P_{j l}, \overrightarrow{\nu_{j l}}\right\rangle & \geqslant-6 r_{j} \delta+\alpha r_{j}(1-2 \sqrt{\delta})-C_{n} \alpha r_{j} \sqrt{\alpha} \\
& \geqslant r_{j}\left(\alpha(1-3 \sqrt{\alpha})-C_{n} \alpha \sqrt{\alpha}-6 \delta\right) .
\end{aligned}
$$

Choosing $\alpha$ so that $1-3 \sqrt{\alpha}>\frac{1}{2}$ and $C_{n} \alpha<\frac{1}{4}$, and $\delta>0$ so that $\delta<\frac{\alpha}{48}$ we conclude that

$$
\left\langle\phi_{j}(X)-P_{j l}, \overrightarrow{\nu_{j l}}\right\rangle \geqslant \frac{\alpha}{4} r_{j}-6 \delta r_{j} \geqslant 6 \delta r_{j}>2 \delta(1+2 \sqrt{\alpha}) r_{j},
$$

which implies that $X_{j}=\phi_{j}(X) \in \Omega$ by (1.9). Hence we have shown that

$$
\bar{\Omega}_{j} \cap B\left(Q_{0}, \frac{3 R}{2}\right) \subset \Omega \cap B\left(Q_{0}, \frac{3 R}{2}\right) .
$$

In order to prove (A.1.45) and (A.1.46) we need to look at the Jacobian of $\phi_{j}$ on $\partial \Omega, J_{\partial \Omega} \phi_{j}$. If $P \in \partial^{*} \Omega$, let $\tau_{1}(P), \ldots, \tau_{n}(P)$ be an orthonormal basis for $T_{P} \partial \Omega$. Note that for $k=1, \ldots, n$

$$
D \phi_{j}(P)\left(\tau_{k}(P)\right)=\tau_{k}(P)+\alpha r_{j} \sum_{i}\left\langle\nabla \lambda_{j i}(P), \tau_{k}(P)\right\rangle \overrightarrow{n_{j i}}
$$


where $\nabla \lambda_{j i}(P)$ denotes the gradient of $\lambda_{j i}$ in $\mathbb{R}^{n+1}$, and $D \phi_{j}(P): T_{P} \partial \Omega \rightarrow \mathbb{R}^{n+1}$ is the linear map induced by $\phi_{j}$ on $T_{P} \partial \Omega$. By definition (see $[26, \S 12]$ )

$$
J \phi_{j}(P)=J_{\partial \Omega} \phi_{j}(P)=\sqrt{\operatorname{det} D \phi_{j}(P)^{*} \circ D \phi_{j}(P)}
$$

where $D \phi_{j}(P)^{*}: \mathbb{R}^{n+1} \rightarrow T_{P} \partial \Omega$ denotes the adjoint transformation to $D \phi_{j}(P)$.

Since

$$
\begin{aligned}
D \phi_{j}(P)^{*} \circ D \phi_{j}(P)\left(\tau_{l}(P)\right) & =\sum_{k=1}^{n}\left\langle D \phi_{j}(P)^{*} \circ D \phi_{j}(P)\left(\tau_{k}(P)\right), \tau_{l}(P)\right\rangle \tau_{k}(P) \\
& =\sum_{k=1}^{n}\left\langle D \phi_{j}(P)\left(\tau_{k}(P)\right), D \phi_{j}(P)\left(\tau_{l}(P)\right)\right\rangle \tau_{k}(P)
\end{aligned}
$$

then by 1.6 .4 and 1.7.5 in [8]

$$
\left(J \phi_{j}(P)\right)^{2}=\left\langle D \phi_{j}(P)^{*} \circ D \phi_{j}(P)\left(\tau_{1}(P)\right) \wedge \cdots \wedge D \phi_{j}(P)^{*} \circ D \phi_{j}(P)\left(\tau_{n}(P)\right),\right.
$$

$$
\left.\tau_{1}(P) \wedge \cdots \wedge \tau_{n}(P)\right\rangle
$$

where $\langle$,$\rangle denotes the inner product in \Lambda_{n} T_{p} \partial \Omega$ induced by that of $T_{p} \partial \Omega$. By 1.7.5 in [8] and [3, Chapter 1], if $\varphi_{l}, \omega_{l}: T_{P} \partial \Omega \rightarrow \mathbb{R}$ denote the 1-forms defined by $\omega_{l}(v)=\left\langle D \phi_{j}(P)\left(\tau_{l}(P)\right), v\right\rangle$ and $\varphi_{l}(v)=\left\langle D \phi_{j}(P)^{*} \circ D \phi_{j}(P)\left(\tau_{l}(P)\right), v\right\rangle$. Then

$$
\text { (A.1.69) } \begin{aligned}
\langle D & \phi_{j}(P)^{*} \circ D \phi_{j}(P)\left(\tau_{1}(P)\right) \wedge \cdots \wedge D \phi_{j}(P)^{*} \\
& \left.\circ D \phi_{j}(P)\left(\tau_{n}(P)\right), \tau_{1}(P) \wedge \cdots \wedge \tau_{n}(P)\right\rangle \\
= & \left\langle\varphi_{1} \wedge \cdots \wedge \varphi_{n}\right\rangle\left(\tau_{1}(P), \ldots, \tau_{n}(P)\right) \\
= & \operatorname{det}\left\langle\varphi_{l}\left(\tau_{k}(P)\right\rangle=\operatorname{det}\left\langle D \phi_{j}(P)^{*} \circ D \phi_{j}(P)\left(\tau_{l}(P)\right), \tau_{n}(p)\right\rangle\right. \\
= & \operatorname{det}\left\langle D \phi_{j}(P)\left(\tau_{l}(P)\right), D \phi_{j}(P)\left(\tau_{n}(P)\right)\right\rangle=\operatorname{det}\left(\omega_{l}\left(D \phi_{j}(P)\left(\tau_{k}(P)\right)\right)\right) \\
= & \left\langle\omega_{1} \wedge \cdots \wedge \omega_{n}\right\rangle\left(D \phi_{j}\left(\tau_{1}(P)\right), \ldots, D \phi_{j}\left(\tau_{n}(P)\right)\right) \\
= & \left\langle D \phi_{j}(P)\left(\tau_{1}(P)\right) \wedge \cdots \wedge D \phi_{j}(P)\left(\tau_{n}(P)\right), D \phi_{j}\left(\tau_{1}(P)\right) \wedge \cdots \wedge D \phi_{j}\left(\tau_{k}(P)\right)\right\rangle \\
= & \left|D \phi_{j}(P)\left(\tau_{1}(P)\right) \wedge \cdots \wedge D \phi_{j}\left(\tau_{n}(P)\right)\right|^{2} .
\end{aligned}
$$

Combining (A.1.68) and (A.1.69) we conclude that

$$
J \phi_{j}(P)=\left|D \phi_{j}(P)\left(\tau_{1}(P)\right) \wedge \cdots \wedge D \phi_{j}(P)\left(\tau_{n}(P)\right)\right| .
$$

Since $\Omega$ is a set of locally finite perimeter whose measure theoretic boundary corresponds to its topological boundary then for every $X \in C_{c}^{1}\left(\mathbb{R}^{n+1}, \mathbb{R}^{n+1}\right)$

$$
\int_{\Omega} \operatorname{div} X d x=-\int_{\partial \Omega}\langle X, \vec{n}\rangle d \mathcal{H}^{n},
$$

where $\vec{n}$ is the inner unit normal.

Thus for any $\omega \in \mathcal{D}^{n}\left(\mathbb{R}^{n+1}\right)$ (i.e., $\omega$ is a smooth $n$-form with compact support)

$$
T(\omega)=\int_{\partial \Omega}\langle\omega(Q), \vec{n}(Q)\rangle d \mathcal{H}^{n}
$$


defines an integer multiplicity rectifiable $n$-current $T$. Here $\langle$,$\rangle denotes the usual pairing for$ $\Lambda^{n}\left(\mathbb{R}^{n+1}\right)$ and $\Lambda_{n}\left(\mathbb{R}^{n+1}\right)$. See [26, §27] for notation and details. In this case for $P \in \partial^{*} \Omega$, $\vec{n}(P)= \pm \tau_{1}(P) \wedge \cdots \wedge \tau_{n}(P)$. In particular $\left|\tau_{1}(P) \wedge \cdots \wedge \tau_{n}(P)\right|=1$. Since $\phi_{j}: \mathbb{R}^{n+1} \rightarrow \mathbb{R}^{n+1}$ is a bilipschitz map and $\phi_{j}(\partial \Omega)=\partial \Omega_{j}, T_{j}=\left(\phi_{j}\right)_{\#} T$ defines an integer multiplicity rectifiable $n$-current, namely

$$
T_{j}(\omega)=\int_{\partial \Omega_{j}}\left\langle\omega\left(\phi\left(Q_{j}\right)\right), \vec{n}_{j}\left(Q_{j}\right)\right\rangle d \mathcal{H}^{n}\left(Q_{j}\right)
$$

where $\overrightarrow{n_{j}}\left(P_{j}\right)=\frac{D \phi_{j}(P)_{\#} \vec{n}(P)}{\left|J \phi_{j}(P)\right|}$ if $\phi_{j}(P)=P_{j}$ and $P \in \partial^{*} \Omega$ (i.e., for $\mathcal{H}^{n}$ a.e. $\left.P_{j} \in \partial^{*} \Omega_{j}\right)$. By $\S 26$ in [26] and the remark above we have that

$$
\begin{aligned}
\overrightarrow{n_{j}}\left(P_{j}\right) & =\frac{D \phi_{j}(P)_{\#} \vec{n}(P)}{\left|J \phi_{j}(P)\right|} \\
& = \pm \frac{D \phi_{j}(P)\left(\tau_{1}(P)\right) \wedge \cdots \wedge D \phi_{j}(P)\left(\tau_{n}(P)\right)}{\left|J \phi_{j}(P)\right|} .
\end{aligned}
$$

Hence in order to understand the behavior of $J \phi_{j}$ and $\overrightarrow{n_{j}}$ as $j$ tends to infinity we need to analyze the behavior of $D \phi_{j}(P)\left(\tau_{l}(P)\right)$ for $P \in \partial^{*} \Omega$ and $l=1, \ldots, n$.

First note that since $\phi_{j}: \mathbb{R}^{n+1} \rightarrow \mathbb{R}^{n+1}$ is bilipschitz, $\mathcal{H}^{n}\left(\partial \Omega \backslash \partial^{*} \Omega\right)=\mathcal{H}^{n}\left(\partial \Omega_{j} \backslash \partial^{*} \Omega_{j}\right)=0$ by Lemma A.1.1 and Remark 4.2 in [18], and $\phi_{j}(\partial \Omega)=\partial \Omega_{j}$ then

$$
\mathcal{H}^{n}\left(\partial^{*} \Omega_{j} \backslash \phi_{j}\left(\partial^{*} \Omega\right)\right)=\mathcal{H}^{n}\left(\partial^{*} \Omega \backslash \phi_{j}^{-1}\left(\partial^{*} \Omega_{j}\right)\right)=0 .
$$

For $P \in \partial^{*} \Omega \cap B\left(Q_{0}, 2 R\right)$ and $l=1, \ldots, n$, since $\sum_{i} \nabla \lambda_{j i}(P)=0$ then

$$
\begin{aligned}
\sum_{i}\left\langle\nabla \lambda_{j i}(P), \tau_{l}(P)\right\rangle \overrightarrow{n_{j i}} & =\sum_{i}\left\langle\nabla \lambda_{j i}(P), \tau_{l}(P)\right\rangle\left(\overrightarrow{n_{j i}}-\vec{n}(P)\right) \\
& =\sum_{\left|P-P_{j i}\right| \leqslant \frac{13}{4} r_{j}}\left\langle\nabla \lambda_{j i}(P), \tau_{l}(P)\right\rangle\left(\overrightarrow{n_{j i}}-\vec{n}(P)\right) \\
& \leqslant C_{n} \sup _{\left|P-P_{j i}\right| \leqslant \frac{13}{4} r_{j}}\left|\overrightarrow{n_{j i}}-\vec{n}(P)\right|,
\end{aligned}
$$

where $\vec{n}(P)$ denotes the inner unit normal to $\partial \Omega$ at $P$. Thus for $P \in \partial^{*} \Omega$ and $l=1,2, \ldots, n$,

$$
\left|D \phi_{j}\left(\tau_{l}(P)\right)-\tau_{l}(P)\right| \leqslant C_{n} \alpha \sup _{\left|P-P_{j i}\right| \leqslant \frac{13}{4} r_{j}}\left|\overrightarrow{n_{j i}}-\vec{n}(P)\right| .
$$

\section{LEMmA A.1.3. - Using the notation above we claim that for $P \in \partial^{*} \Omega$}

$$
\lim _{j \rightarrow \infty} \sup _{\left|P-P_{j i}\right| \leqslant \frac{13}{4} r_{j}}\left|\overrightarrow{n_{j i}}-\vec{n}(P)\right|=0 .
$$

We postpone the proof of this lemma until later, and continue with the proof of Lemma A.1.2.

Combining (A.1.77) and (A.1.78) we conclude that for $P \in \partial^{*} \Omega$

$$
\lim _{j \rightarrow \infty}\left|D \phi_{j}\left(\tau_{l}(P)\right)-\tau_{l}(P)\right|=0
$$

Since $P \in \partial^{*} \Omega$ by $\S 1.7 .5$ in [8], (A.1.77) and (A.1.78) we have that

$4^{\mathrm{e}}$ SÉRIE - TOME $36-2003-\mathrm{N}^{\circ} 3$ 
(A.1.80)

$$
\begin{aligned}
\mid D \phi_{j} & \left(\tau_{1}(P)\right) \wedge \cdots \wedge D \phi_{j}\left(\tau_{n}(P)\right)-\tau_{1}(P) \wedge \cdots \wedge \tau_{n}(P) \mid \\
\leqslant & \mid D \phi_{j}\left(\tau_{1}(P)\right) \wedge \cdots \wedge D \phi_{j}\left(\tau_{n}(P)\right) \\
& -D \phi_{j}\left(\tau_{1}(P)\right) \wedge \cdots \wedge D \phi_{j}\left(\tau_{n-1}(P)\right) \wedge \tau_{n}(P) \mid \\
& +\mid D \phi_{j}\left(\tau_{1}(P)\right) \wedge \cdots \wedge D \phi_{j}\left(\tau_{n-1}(P)\right) \wedge \tau_{n}(P) \\
& \quad-D \phi_{j}\left(\tau_{1}(P)\right) \wedge \cdots \wedge D \phi_{j}\left(\tau_{n-1}(P)\right) \wedge \tau_{n-1}(P) \wedge \tau_{n}(P) \mid+\cdots \\
& +\left|D \phi_{j}\left(\tau_{1}(P)\right) \wedge \tau_{2}(P) \wedge \cdots \wedge \tau_{n}(P)-\tau_{1}(P) \wedge \cdots \wedge \tau_{n}(P)\right| \\
\leqslant & \sum_{i=1}^{n} \mid D \phi_{j}\left(\tau_{1}(P)\right) \wedge \cdots \wedge D \phi_{j}\left(\tau_{i-1}(P)\right) \wedge\left(D \phi_{j}\left(\tau_{i}(P)\right)\right. \\
& \left.-\tau_{i}(P)\right) \wedge \tau_{i+1}(P) \wedge \cdots \wedge \tau_{n}(P) \mid \\
\leqslant & \sum_{i=1}^{n}\left|D \phi_{j}\left(\tau_{1}(P)\right)\right| \cdots\left|D \phi_{j}\left(\tau_{i-1}(P)\right)\right|\left|D \phi_{j}\left(\tau_{i}(P)\right)-\tau_{i}(P)\right| \\
& \times\left|\tau_{i+1}(P)\right| \cdots\left|\tau_{n}(P)\right| \\
\leqslant & C_{n} \alpha \sup _{\left|P-P_{j i}\right| \leqslant \frac{13}{4} r_{j}}\left|\overrightarrow{n_{j i}}-\vec{n}(P)\right| \leqslant C_{n} \alpha .
\end{aligned}
$$

Therefore for $P \in \partial^{*} \Omega$

$$
\lim _{j \rightarrow \infty} D \phi_{j}\left(\tau_{1}(P)\right) \wedge \cdots \wedge D \phi_{j}\left(\tau_{n}(P)\right)=\tau_{1}(P) \wedge \cdots \wedge \tau_{n}(P)
$$

which implies using (A.1.70) and (A.1.74) that for $P \in \partial^{*} \Omega$ and $P_{j}=\phi_{j}(P)$

$$
\lim _{j \rightarrow \infty} J \phi_{j}(P)=1
$$

and

$$
\lim _{j \rightarrow \infty} \overrightarrow{n_{j}}\left(P_{j}\right)=\vec{n}(P) .
$$

This proves (A.1.46). Since $\phi_{j}: \partial \Omega \rightarrow \mathbb{R}^{n+1}$ is a bilipschitz map and $\partial \Omega_{j}=\phi_{j}(\partial \Omega)$ by (A.1.19), the area formula implies (see [26, §8 and §12]) that for any measurable set $A \subset \partial \Omega$

$$
\mathcal{H}^{n}\left(\phi_{j}(A)\right)=\int_{A} J \phi_{j}(Q) d \mathcal{H}^{n}(Q),
$$

and any measurable function on $\partial \Omega, g$,

$$
\int_{\partial \Omega} g J \phi_{j} d \mathcal{H}^{n}=\int_{\partial \Omega_{j}} g\left(\phi_{j}^{-1}(X)\right) d \mathcal{H}^{n}(X) .
$$

(A.1.45) follows from (A.1.85). This concludes the proof of Lemma A.1.2.

Proof of Lemma A.1.3. - Let $P \in \partial^{*} \Omega$, and let $\vec{n}(P)$ denote the inward pointing unit normal vector to $\partial \Omega$. Define
(A.1.86)
$H^{+}(P)=\left\{Y \in \mathbb{R}^{n+1}:\langle\vec{n}(P), Y-P\rangle \geqslant 0\right\}$,
(A.1.87)
$H^{-}(P)=\left\{Y \in \mathbb{R}^{n+1}:\langle\vec{n}(P), Y-P\rangle \leqslant 0\right\}$. 
By Corollary 1 in Section 5.7 in [7] we have that

$$
\lim _{r \rightarrow 0} \frac{\mathcal{H}^{n+1}\left(B(P, r) \cap \Omega \cap H^{-}(P)\right)}{r^{n+1}}=0
$$

and

$$
\lim _{r \rightarrow 0} \frac{\mathcal{H}^{n+1}\left(B(P, r) \cap \Omega^{c} \cap H^{+}(P)\right)}{r^{n+1}}=0 .
$$

We shall prove that given $\varepsilon>0$ there is $r>0$ so that if $Q \in \partial \Omega \cap B(P, r)$ then

$$
\left|\left\langle\vec{n}(P), \frac{Q-P}{|Q-P|}\right\rangle\right| \leqslant \varepsilon
$$

Our proof proceeds by contradiction. First assume that there is $\varepsilon \in(0,1)$ so that for each $m \in \mathbb{N}$, there is $Q_{m} \in \partial \Omega \cap B\left(P, \frac{1}{m}\right)$ so that $\left\langle\vec{n}(P), Q_{m}-P\right\rangle \geqslant \varepsilon\left|Q_{m}-P\right|$ then

$$
B\left(Q_{m}, \varepsilon\left|P-Q_{m}\right|\right) \subset H^{+}(P) \cap B\left(P, 2\left|P-Q_{m}\right|\right)
$$

and

$$
B\left(Q_{m}, \varepsilon\left|P-Q_{m}\right|\right) \cap \Omega^{c} \subset H^{+}(P) \cap \Omega^{C} \cap B\left(P, 2\left|P-Q_{m}\right|\right) .
$$

Since $\Omega^{c}$ satisfies the corkscrew condition for every $m \in \mathbb{N}$

$$
\frac{\mathcal{H}^{n+1}\left(B\left(Q_{m}, \varepsilon\left|P-Q_{m}\right|\right) \cap \Omega^{c}\right)}{\left|P-Q_{m}\right|^{n+1}} \geqslant C_{n} \varepsilon^{n+1} .
$$

On the other hand (A.1.89) implies that

$$
\lim _{m \rightarrow \infty} \frac{\mathcal{H}^{n+1}\left(H^{+}(P) \cap \Omega^{c} \cap B\left(P, 2\left|P-Q_{m}\right|\right)\right.}{\left|P-Q_{m}\right|^{n+1}}=0 .
$$

Thus combining (A.1.92), (A.1.93) and (A.1.94) we obtain a contradiction. Thus given $\varepsilon>0$ there is $r_{1}>0$ so that if $Q \in \partial \Omega \cap B\left(P, r_{1}\right)$ then $\langle\vec{n}(P), P-Q\rangle<\varepsilon|P-Q|$. In a similar way we prove that there exist $r_{2}>0$ so that if $Q \in \partial \Omega \cap B\left(P, r_{2}\right)$ then $\langle\vec{n}(P), P-Q\rangle>-\varepsilon|P-Q|$. Therefore given $\varepsilon>0$ there exists $r_{0}>0$ so that for $r<r_{0}$

$$
\frac{1}{r} \sup _{Q \in \partial \Omega \cap B(P, r)} \operatorname{dist}\left(Q, T_{P} \partial \Omega \cap B(P, r)\right)<\varepsilon .
$$

Since $\partial \Omega$ is $\delta$-Reifenberg flat, combining (1.8) and (A.1.95) we have that for $r<r_{0}$

$$
\frac{1}{r} D\left[\partial \Omega \cap B(P, r), T_{P} \partial \Omega \cap B(P, r)\right] \leqslant 4 \delta+\varepsilon .
$$

Since $\Omega$ satisfies the separation property from (A.1.96) we deduce that for $X \in T_{P} \partial \Omega \cap B(P, r)$ and $r<\frac{r_{0}}{2}$ there exists $Q \in \partial \Omega \cap B(P, r)$ so that if $\Pi$ denotes the orthogonal projection from $\mathbb{R}^{n+1}$ onto $T_{P} \partial \Omega \Pi(Q)=X$, which implies

$$
|Q-X|=|\langle Q-X, \vec{n}(P)\rangle|=|\langle Q-P, \vec{n}(P)\rangle|<\varepsilon|P-Q| \leqslant \varepsilon r .
$$


Combining (A.1.95) and (A.1.97) we conclude that given $\varepsilon>0$ there exists $s>0$ so that for $r<s$

$$
\frac{1}{r} D\left[\partial \Omega \cap B(P, r), T_{P} \partial \Omega \cap B(P, r)\right]<\varepsilon .
$$

Let $j \geqslant 1$ be large enough so that $5 r_{j}<s$, and let

$$
\left|P_{j i}-P\right|<\frac{13}{4} r_{j}
$$

there exists $X_{j i} \in T_{P} \partial \Omega \cap B\left(P, \frac{13}{4} r_{j}\right)$ so that

$$
\left|P_{j i}-X_{j i}\right|<\varepsilon r_{j} .
$$

Let $Q \in \partial \Omega \cap B\left(P_{j i}, r_{j}\right) \subset \partial \Omega \cap B\left(P, \frac{17}{4} r_{j}\right)$, there is $X \in T_{P} \partial \Omega \cap B\left(P, \frac{17}{4} r_{j}\right)$ so that $|Q-X|<\varepsilon \frac{17}{4} r_{j}$. Note that

$$
Y=X-X_{j i}+P_{j i} \in T_{P} \partial \Omega-X_{j i}+P_{j l}, \quad|Q-Y|<\frac{21}{4} \varepsilon r_{j},
$$

and either

$$
\left|Y-P_{j i}\right|<r_{j} \quad \text { or } \quad r_{j} \leqslant\left|Y-P_{j i}\right|=\left|X-X_{j i}\right| \leqslant|X-Q|+\left|Q-P_{j i}\right| \leqslant\left(1+\frac{17}{4} \varepsilon\right) r_{j} .
$$

If $\left|Y-P_{j i}\right|<r_{j}$ let

$$
\begin{gathered}
Z=\left(1-5 \varepsilon \frac{r_{j}}{\left|Y-P_{j i}\right|}\right)\left(Y-P_{j i}\right)+P_{j i}, \quad Z \in T_{P} \partial \Omega-X_{j i}+P_{j i}, \\
|Z-Q| \leqslant|Z-Y|+|Y-Q| \leqslant 5 \varepsilon r_{j}+\frac{21}{4} \varepsilon r_{j}=\frac{41}{4} \varepsilon r_{j},
\end{gathered}
$$

and

$$
\left|Z-P_{j i}\right|=\left|1-5 \varepsilon \frac{r_{j}}{\left|Y-P_{j i}\right|}\right| r_{j}\left(1+\frac{17}{4} \varepsilon\right) \leqslant(1-5 \varepsilon)\left(1+\frac{17}{4} \varepsilon\right) r_{j}<r_{j}
$$

for $\varepsilon>0$ small enough. Hence we have shown that for $Q \in \partial \Omega \cap B\left(P_{j i}, r_{j}\right)$ there exists $Z \in\left(T_{P} \partial \Omega-X_{j i}+P_{j i}\right) \cap B\left(P_{j i}, r_{j}\right)$ and such that $|Q-Z|<11 \varepsilon r_{j}$. The same argument used to prove (A.1.98) ensures that for $\left|P_{j i}-P\right|<\frac{13}{4} r_{j}$ and $5 r_{j}<s$

$$
\frac{1}{r_{j}} D\left[\partial \Omega \cap B\left(P_{j i}, r_{j}\right),\left(T_{P} \partial \Omega-X_{j i}+P_{j i}\right) \cap B\left(P_{j i}, r_{j}\right)\right] \leqslant 11 \varepsilon .
$$

Since $L\left(P_{j i}, r_{j}\right)$ is defined to be the best approximating plane to $\partial \Omega$ at $P_{j i}$ at radius $r_{j}$ we deduce from (A.1.99) that for $\varepsilon>0$ small enough and $j$ large enough depending on $\varepsilon>0$, if $\left|P-P_{j i}\right| \leqslant \frac{13}{4} r_{j}$ then $\left|\overrightarrow{n_{j i}}-\vec{n}(P)\right| \leqslant C_{n} \varepsilon$. Hence

$$
\lim _{j \rightarrow \infty} \sup _{\left|P-P_{j i}\right| \leqslant \frac{13}{4} r_{j}}\left|\overrightarrow{n_{j i}}-\vec{n}(P)\right|=0,
$$

which concludes the proof of Lemma A.1.3. 
This also concludes the construction of the sequence of good approximating domains for Reifenberg flat chord arc domains.

Proposition A.1.1. - Let $\Omega \subset \mathbb{R}^{n+1}$ be a $\delta$-Reifenberg flat chord arc domain. Let $u$ denote the Green's function of $\Omega$, and let $h$ denote the corresponding Poisson kernel. Assume that $h \in L_{\mathrm{loc}}^{2}(d \sigma)$. Let $F$ be the non-tangential limit of $\nabla u, F \in L_{\mathrm{loc}}^{1}\left(d \omega^{X}\right)$ for $X \in \Omega$. Then $\mathcal{H}^{n}$ a.e. $Q \in \partial \Omega$

(A.1.100)

$$
h(Q)=\langle F(Q), \vec{n}(Q)\rangle
$$

where $\vec{n}$ denote the inward pointing unit normal.

Proof of Proposition A.1.1. - We prove that (A.1.100) holds for $\mathcal{H}^{n}$ a.e. $Q \in \partial \Omega$ by showing that it holds for $\mathcal{H}^{n}$ a.e. $Q \in \partial \Omega \cap K$, for any compact set $K \subset \mathbb{R}^{n+1}$. We do this by showing that for such $K \subset \mathbb{R}^{n+1}$ there exists $R>0$ so that (A.1.100) holds for $\mathcal{H}^{n}$ a.e. $Q \in \partial \Omega \cap B\left(Q_{0}, R\right)$ when $Q_{0} \in K$. For $K \subset \mathbb{R}^{n+1}$, let $R>0$ be as chosen at the beginning of the appendix (and so that $A \notin B(Q, 4 R)$ if necessary), let $Q_{0} \in \partial \Omega \cap K$, and let $\varphi \in C_{c}^{\infty}\left(B\left(Q_{0}, R\right)\right)$. (A.1.20) ensures that

$$
\int_{\partial \Omega} \varphi(Q) h(Q) d \mathcal{H}^{n}=\int_{\Omega} u \Delta \varphi=\lim _{j \rightarrow \infty} \int_{\Omega_{j}} u \Delta \varphi .
$$

Since $\Omega_{j} \cap B\left(Q_{0}, \frac{3 R}{2}\right) \subset \Omega \cap B\left(Q_{0}, \frac{3 R}{2}\right) u$ is harmonic on $\Omega_{j} \cap B\left(Q_{0}, \frac{3 R}{2}\right)$, Green's theorem ensures that

$$
\int_{\Omega_{j}} u \Delta \varphi=\int_{\partial \Omega_{j}}\left(\varphi\left\langle\nabla u, \overrightarrow{n_{j}}\right\rangle-u\left\langle\nabla \varphi, \overrightarrow{n_{j}}\right\rangle\right) d \mathcal{H}^{n} .
$$

By (A.1.42) we know that if $Q_{j} \in \partial \Omega_{j} \operatorname{dist}\left(Q_{j}, \partial \Omega\right) \leqslant \alpha K_{n} r_{j}$, which implies by Lemma 4.1 in [14] that

$$
u\left(Q_{j}\right) \leqslant c\left(\frac{r_{j}}{R}\right)^{\alpha} \sup _{B\left(Q, \frac{3 R_{0}}{2}\right)} u .
$$

Thus

$$
\lim _{j \rightarrow \infty} \int_{\partial \Omega_{j}} u\left\langle\nabla \varphi, \overrightarrow{n_{j}}\right\rangle d \mathcal{H}^{n}=0 .
$$

Since $\phi_{j}$ is a smooth bilipschitz map on $\mathbb{R}^{n+1}$ and $\overrightarrow{n_{j}}$ is a measurable function on $\phi_{j}(\partial \Omega)=\partial \Omega_{j}$ then $\overrightarrow{n_{j}} \circ \phi_{j}$ is a measurable function on $\partial \Omega$ and (A.1.85) implies that

$$
\begin{aligned}
& \int_{\partial \Omega_{j}} \varphi\left\langle\nabla u, \overrightarrow{n_{j}}\right\rangle d \mathcal{H}^{n} \\
& \quad=\int_{\partial \Omega} \varphi\left(\phi_{j}(Q)\right)\left\langle\nabla u\left(\phi_{j}(Q)\right), \overrightarrow{n_{j}}\left(\phi_{j}(Q)\right)\right\rangle J \phi_{j}(Q) d \mathcal{H}^{n}(Q) .
\end{aligned}
$$

Note that by (A.1.40) if $Q \in B\left(Q_{0}, R\right)$

$$
\operatorname{dist}\left(\phi_{j}(Q), \partial \Omega\right) \leqslant\left|\phi_{j}(Q)-Q\right| \leqslant K_{n} r_{j} .
$$

By (A.1.63) there exists $P_{j l} \in \partial \Omega$ so that $\left|Q-P_{j l}\right| \leqslant r_{j}(1+2 \sqrt{\alpha})$ and $\left\langle\phi_{j}(Q)-P_{j l}, \overrightarrow{\nu_{j l}}\right\rangle \geqslant \frac{\alpha}{4} r_{j}$ where $\overrightarrow{\nu_{j l}}=\vec{n}\left(P_{j l},(1+2 \sqrt{\alpha}) r_{j}\right)$. Let $\alpha$ be so that $2 \sqrt{\alpha}<\frac{1}{2}$. If $\overrightarrow{\eta_{j l}}=\vec{n}\left(P_{j l}, 2 r_{j}\right)$ then 
$\left|\overrightarrow{\nu_{j l}}-\overrightarrow{\eta_{j l}}\right| \leqslant C_{n} \delta$, and for $\delta>0$ small with respect to $\alpha>0$

(A.1.107)

$$
\left\langle\phi_{j}(Q)-P_{j l}, \overrightarrow{\eta_{j l}}\right\rangle \geqslant \frac{\alpha}{8} r_{j}
$$

Combining (1.9) and (1.10) we have that

$$
\partial \Omega \cap B\left(P_{j l}, 2 r_{j}\right) \subset\left\{x \in B\left(P_{j l}, 2 r_{j}\right),\left|\left\langle x-P_{j l}, \overrightarrow{\eta_{j l}}\right\rangle\right| \leqslant 4 \delta\right\} .
$$

From (A.1.107) and (A.1.108) we deduce that

$$
d\left(\phi_{j}(Q), \partial \Omega \cap B\left(P_{j l}, 2 r_{j}\right)\right) \geqslant\left(\frac{\alpha}{8}-4 \delta\right) r_{j} .
$$

Since $Q \in B\left(P_{j l}, \frac{3}{2} r_{j}\right)$, and $\alpha<\frac{1}{2}$ we conclude from (A.1.106) and (A.1.109) that for $\delta$ small enough $4 \delta<\frac{\alpha}{16}$

$$
\text { (A.1.110) } \frac{\alpha}{16} r_{j} \leqslant\left|\phi_{j}(Q)-Q\right| \leqslant K_{n} r_{j} \quad \text { and } \quad \frac{\alpha r_{j}}{16} \leqslant \operatorname{dist}\left(\phi_{j}(Q), \partial \Omega\right) \leqslant K_{n} r_{j} \text {. }
$$

Thus $\phi_{j}(Q)$ approaches $Q$ non-tangentially as $j \rightarrow \infty$, in particular $\phi_{j}(Q) \in \Gamma_{\beta}(Q)$ for $\beta=16 K_{n} / \alpha$, where $\Gamma_{\beta}(Q)=\{X \in \Omega:|X-Q| \leqslant \beta \operatorname{dist}(X, \partial \Omega)\}$. Hence using the result in Lemma 3.1, (A.1.46), (A.1.82) and (A.1.110) we have that for $\mathcal{H}^{n}$ a.e. $Q \in \partial \Omega$

$$
\text { (A.1.111) } \varphi\left(\phi_{j}(Q)\right)\left\langle\nabla u\left(\phi_{j}(Q)\right), \vec{n}_{j}\left(\phi_{j}(Q)\right)\right\rangle J \phi_{j}(Q) \underset{j \rightarrow \infty}{\longrightarrow} \varphi(Q)\langle F(Q), \vec{n}(Q)\rangle .
$$

Since

$$
\sup _{X \in \Gamma_{\beta}(Q), \delta(X) \leqslant \ell}|\nabla u(X)| \leqslant C M_{\ell}(h)(Q)
$$

where $M_{\ell}(h) \in L_{\text {loc }}^{2}(d \sigma)$ (see proof of Lemma 3.1), and $J \phi_{j}(Q) \leqslant 1+C_{n} \alpha$ by (A.1.80), the Lebesgue dominated convergence theorem ensures that

$$
\int_{\partial \Omega} \varphi\left(\phi_{j}(Q)\right)\left\langle\nabla u\left(\phi_{j}(Q)\right), \overrightarrow{n_{j}}\left(\phi_{j}(Q)\right)\right\rangle J \phi_{j}(Q) d \mathcal{H}^{n}(Q)
$$

$$
\underset{j \rightarrow \infty}{\longrightarrow} \int_{\partial \Omega} \varphi(Q)\langle F(Q), \vec{n}(Q)\rangle .
$$

Combining (A.1.101), (A.1.102), (A.1.104), (A.1.105) and (A.1.112) we have that for all $\varphi \in C_{c}^{\infty}\left(B\left(Q_{0}, R\right)\right)$

$$
\int_{\partial \Omega} \varphi(Q) h(Q) d \mathcal{H}^{n}(Q)=\int_{\partial \Omega} \varphi(Q)\langle F(Q), \vec{n}(Q)\rangle d \mathcal{H}^{n}(Q),
$$

which implies that for $\mathcal{H}^{n}$ a.e. $Q \in \partial \Omega \cap B\left(Q_{0}, R\right)$

$$
h(Q)=\langle F(Q), \vec{n}(Q)\rangle .
$$

This concludes the proof of Proposition A.1.1. 


\section{A.2. Behavior of the tangential components of non-tangential limits}

The goal of this section is to show that almost everywhere on $\partial \Omega$ the tangential components of $F$ (the non-tangential limit of $\nabla u$, where $u$ denotes either the Green's function with pole at infinity or with pole at $A$ ) are zero. The original proof of this fact made use of the parameterizations for chord-arch surfaces with small constant constructed by Semmes in [24]. In conversations with G. David he pointed out that there was a much simpler proof. The proof presented here is due to him. The authors are very grateful to him for this contribution. Before starting the proof we need to specify the properties satisfied by the points $Q \in \partial^{*} \Omega$ for which we can prove that $F(Q)-\langle F(Q), \vec{n}(Q)\rangle \vec{n}(Q)=0$. Recall that for $\mathcal{H}^{n}$ a.e. $Q \in \partial^{*} \Omega, \nabla u$ converges non-tangentially to $F(Q)$; i.e.

$$
F(Q)=\lim _{\substack{X \rightarrow Q \\ X \in \Gamma(Q)}} \nabla u(X)
$$

here $\Gamma(Q)$ denotes a non-tangential cone with vertex $Q$. (We do not specify the "angle" since it does not play a role.) Let $l$ be 1 if $u$ is the Green's function with pole at infinity, and let $l$ be $\frac{\delta(A)}{4}$ if $u$ is the Green's function with pole at $A$. Consider the non-tangential maximal function of $\nabla u$ at $Q$

$$
N_{l} \nabla u(Q)=\sup _{X \in \Gamma(Q), \delta(X) \leqslant l}|\nabla u(X)| \leqslant C M_{l}(h)(Q)
$$

by (3.10). Moreover since $h \in L_{\text {loc }}^{2}(d \sigma)$ then $M_{l}(h) \in L_{\text {loc }}^{2}(d \sigma)$ and so $N_{l} \nabla u \in L_{\text {loc }}^{2}(d \sigma)$ (see (3.12)). Thus for $\mathcal{H}^{n}$ a.e. $Q \in \partial \Omega N_{l} \nabla u(Q)<\infty$ and

$$
\lim _{r \rightarrow 0} \oint_{B(Q, r) \cap \partial \Omega}\left|N_{l} \nabla u(P)-N_{l} \nabla u(Q)\right| d \mathcal{H}^{n}(P)=0
$$

which implies, since $\partial \Omega$ is Ahlfors regular, that for $\mathcal{H}^{n}$ a.e. $Q \in \partial \Omega$

$$
\lim _{r \rightarrow \infty} \frac{1}{r^{n}} \mathcal{H}^{n}\left(\left\{P \in B(Q, r) \cap \partial \Omega: N_{l} \nabla u(P)>2 N_{l} \nabla u(Q)\right\}\right)=0 .
$$

In particular

$$
\lim _{r \rightarrow 0} \delta_{\varepsilon}^{\prime}(r)=0
$$

where we set

$$
\delta_{\varepsilon}^{\prime}(r)=\frac{1}{r^{n}} \mathcal{H}^{n}\left(\left\{P \in B(Q, 2 r) \cap \partial \Omega: N_{l} \nabla u(P)>2 N_{l} \nabla u(Q)\right\}\right) .
$$

Note also that for $\varepsilon>0$ and $\mathcal{H}^{n}$ a.e. $Q \in \partial \Omega$

$$
\lim _{r \rightarrow 0} \delta_{\varepsilon}(r)=0
$$

where we set

$$
\delta_{\varepsilon}(r)=\frac{1}{r^{n}} \mathcal{H}^{n}(\{P \in B(Q, 2 r) \cap \partial \Omega ;|F(P)-F(Q)|>\varepsilon\}) .
$$


In order to do the proof we need to recall the proof of Egoroff's theorem, which asserts that $\nabla u$ converges uniformly to $F$ on large sets. For all $\varepsilon>0$ and $l>0$ define

(A.2.9) $H(l, \varepsilon)=\{P \in \partial \Omega:|F(P)-\nabla u(X)| \leqslant \varepsilon$ for all $X \in \Gamma(Q)$ with $\delta(X) \leqslant l\}$

where the existence of the limit $F(P)$ is part of the definition.

Because of (A.2.1) we know that for each $\varepsilon>0 \mathcal{H}^{n}$ a.e. $Q \in \partial \Omega$ lies in some $H(l, \varepsilon)$. Therefore given any $\eta>0$ we can find $l=l(\varepsilon, \eta)$ so that $\mathcal{H}^{n}(\partial \Omega \backslash H(l(\varepsilon, \eta), \varepsilon))<\eta$. We apply this to $\varepsilon_{n}=2^{-n}, \eta_{n}=2^{-n-1} \eta$, for $\eta>0$. We get small numbers $l_{n}=l\left(2^{-n}, 2^{-n-1} \eta\right)$. Define

$$
E(\eta)=\bigcap_{n \geqslant 1} H\left(l_{n}, 2^{-n}\right)
$$

Then

$$
\mathcal{H}^{n}(\partial \Omega \backslash E(\eta)) \leqslant \sum_{n} \mathcal{H}^{n}\left(\partial \Omega \backslash H\left(l_{n}, 2^{-n}\right)\right) \leqslant \sum_{n} 2^{-n-1} \eta \leqslant \eta,
$$

while (A.2.10) and the definition (A.2.9) ensure uniform convergence of $\nabla u(X)$ in $\Gamma(Q)$ for $Q \in E(\eta)$. Note also that

$$
\mathcal{H}^{n}\left(\partial \Omega \backslash \bigcup_{\eta>0} E(\eta)\right)=0 .
$$

Thus for $\mathcal{H}^{n}$ a.e. $Q \in \partial^{*} \Omega, Q$ is a density point for some $E(\eta)$. This means that if we set

$$
\delta^{\prime \prime}(r)=\frac{1}{r^{n}} \mathcal{H}^{n}(\partial \Omega \cap B(Q, 2 r) \backslash E(\eta))
$$

then

$$
\lim _{r \rightarrow 0} \delta^{\prime \prime}(r)=0 .
$$

We are now ready to prove the following statement.

PROPOSITION A.2.2. - Let $\Omega \subset \mathbb{R}^{n+1}$ be a $\delta$-Reifenberg flat chord-arc domain. Let $u$ denote the Green's function of $\Omega$ (either with pole at $A$ or at infinity) and let $h$ denote the corresponding Poisson kernel. Assume that $h \in L_{\mathrm{loc}}^{2}(d \sigma)$.

Let $F$ be the non-tangential limit of $\nabla u, F \in L_{\mathrm{loc}}^{1}\left(d \omega^{X}\right)$ for $X \in \Omega$. Then $\mathcal{H}^{n}$ a.e. $Q \in \partial \Omega$

$$
F(Q)=h(Q) \vec{n}(Q)
$$

where $\vec{n}$ denotes the inward pointing unit normal.

Proof. - Given $\varepsilon>0$, let $Q_{0} \in \partial^{*} \Omega$ be such that (A.2.4), (A.1.95), (A.2.7) and (A.2.14) are satisfied. Since $Q_{0} \in \partial^{*} \Omega$, (A.1.95) ensures

$$
\lim _{r \rightarrow \infty} \frac{1}{r} \sup _{Q \in \partial \Omega \cap B\left(Q_{0}, 2 r\right)} \operatorname{dist}\left(Q, T_{Q_{0}} \partial \Omega \cap B\left(Q_{0}, 2 r\right)\right)=0 .
$$

Let $l(r)$ be a non-negative function satisfying

$$
\lim _{r \rightarrow 0} \frac{l(r)}{r}=0
$$




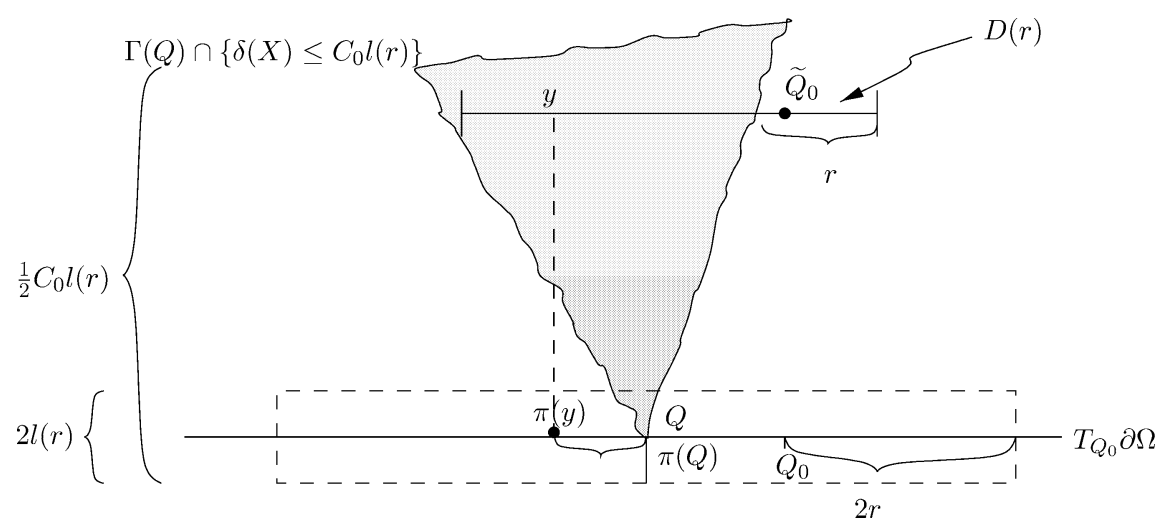

Fig. 2.

$$
\sup _{Q \in \partial \Omega \cap B\left(Q_{0}, 2 r\right)} \operatorname{dist}\left(Q, T_{Q_{0}} \partial \Omega \cap B\left(Q_{0}, 2 r\right)\right) \leqslant l(r),
$$

and

$$
\begin{aligned}
& \mathcal{H}^{n}(\partial \Omega \cap B(Q, l(r))) \geqslant 2\left[\delta_{\varepsilon}(r)+\delta_{\varepsilon}^{\prime}(r)+\delta^{\prime \prime}(r)\right] r^{n} \\
& \quad \text { for all } Q \in \partial \Omega \cap B\left(Q_{0}, 2 r\right) .
\end{aligned}
$$

It is possible to have (A.2.17), (A.2.18) and (A.2.19) simultaneously because (A.2.5), (A.2.7) and (A.2.14) hold and $\partial \Omega$ is Ahlfors regular.

We now define a disc $D(r)$ which is parallel to $T_{Q_{0}} \partial \Omega$, has radius $r$, lies in $\Omega$, "just above $Q_{0}$ " at distance $\frac{1}{2} C_{0} l(r)$ from $Q_{0}$, where $C_{0}$ is a large constant to be specified shortly. By "just above $Q_{0}$ " we mean that if $\pi$ denotes the orthogonal projection onto $T_{Q_{0}} \partial \Omega$ then $\pi\left(\widetilde{Q}_{0}\right)=Q_{0}$ where $\widetilde{Q}_{0}$ is the center of $D(r)$.

The condition on $C_{0}$, (and on the "aperture" of the NTA cones $\Gamma(Q)$ at the same time) is that

$$
\left\{\begin{array}{l}
\text { if } y \in D(r) \text { and } Q \in \partial \Omega \cap B\left(Q_{0}, 2 r\right) \text { are such that } \\
\quad|\pi(y)-\pi(Q)| \leqslant 2 l(r), \text { then } \\
D(r) \cap B(y, l(r)) \subset \Gamma(Q) \cap\left\{X \in \mathbb{R}^{n+1}, \delta(X) \leqslant l\right\} \text { for } l \geqslant C_{0} l(r) .
\end{array}\right.
$$

The general idea of the argument is as follows. We take random points $y_{1}, y_{2}$ of $D(r)$, and estimate $\left\langle F\left(Q_{0}\right), y_{2}-y_{1}\right\rangle$ in terms of the function $u$, and in particular $u\left(y_{2}\right)-u\left(y_{1}\right)$. We will need the following lemma whose proof we postpone until after we finish the proof of Proposition A.2.2.

LEMMA A.2.4. - Under the assumptions above, if $r>0$ is small enough, then

$$
|u(y)| \leqslant C N_{l} \nabla u\left(Q_{0}\right) l(r) \quad \text { for } y \in D(r)
$$

Define for $y_{1}, y_{2} \in D(r)$

$$
R\left(y_{1}, y_{2}\right)=u\left(y_{2}\right)-u\left(y_{1}\right)-\left\langle F\left(Q_{0}\right), y_{2}-y_{1}\right\rangle .
$$


Since

$$
u\left(y_{2}\right)-u\left(y_{1}\right)=\int_{0}^{1}\left\langle\nabla u\left(y_{1}+t\left(y_{2}-y_{1}\right)\right), y_{2}-y_{1}\right\rangle d t
$$

we have that

$$
\left|R\left(y_{1}, y_{2}\right)\right| \leqslant\left|y_{2}-y_{1}\right| \int_{0}^{1}\left|\nabla u\left(y_{1}+t\left(y_{2}-y_{1}\right)\right)-F\left(Q_{0}\right)\right| d t
$$

It is enough to only look at the average

$$
I(r)=\frac{1}{r} \oint_{D(r)} f_{D(r)}\left|R\left(y_{1}, y_{2}\right)\right| d y_{1} d y_{2}
$$

where we integrate against Lebesgue measure. Using Fubini and combining (A.2.24) and (A.2.25) we have that

$$
\begin{aligned}
I(r) \leqslant & \frac{C_{n}}{r^{n+1}} \oint_{D(r)} \int_{\mathbb{S}^{n-1}} \int_{0}^{2 r} \rho^{n-1} \int_{0}^{\rho} \chi_{D(r)}\left(y_{1}+\rho \omega\right) \\
& \times\left|\nabla u(y+s \omega)-F\left(Q_{0}\right)\right| d s d \rho d \omega d y_{1} \\
\leqslant & \frac{C_{n}}{r^{n+1}} \int_{D(r)} \int_{0}^{2 r} \rho^{n-1} \int_{D\left(y_{1}, \rho\right) \cap D(r)} \frac{\left|\nabla u(X)-F\left(Q_{0}\right)\right|}{\left|y_{1}-X\right|^{n-1}} d X d \rho d y_{1} \\
\leqslant & \frac{C_{n}}{r} \int_{D(r)} \int_{D(r)} \frac{\left|\nabla u(X)-F\left(Q_{0}\right)\right|}{\left|y_{1}-X\right|^{n-1}} d X d y_{1} \\
\leqslant & \frac{C_{n}}{r^{n+1}} \int_{D(r)}\left|\nabla u(X)-F\left(Q_{0}\right)\right| \int_{D(r)} \frac{d y_{1}}{\left|X-y_{1}\right|^{n-1}} d X \\
\leqslant & C_{n} \underset{D(r)}{\left|\nabla u(X)-F\left(Q_{0}\right)\right| d X .}
\end{aligned}
$$

In the previous computation $D\left(y_{1}, \rho\right)$ denotes the intersection of the ball $B\left(y_{1}, \rho\right)$ and the plane parallel to $T_{Q_{0}} \partial \Omega$ which lies at distance $\frac{1}{2} C_{0} l(r)$ from $Q_{0}$.

Next we claim that

$$
\left|\nabla u(y)-F\left(Q_{0}\right)\right| \leqslant 2 \varepsilon \quad \text { for } y \in D(r) .
$$

In fact let $y \in D(r)$ be given. Since $\Omega$ is a Reifenberg flat domain using the separation property it is easy to show that there exists $Q(y) \in \partial \Omega \cap B\left(Q_{0}, 2 r\right)$ such that $\pi(Q(y))=\pi(y)$. Let $B(y)=\partial \Omega \cap B(Q(y), l(r))$ with $l(r)$ as before. We want to choose a point $Q \in B(y)$ carefully in order to obtain (A.2.27) by estimating

$$
\left|\nabla u(y)-F\left(Q_{0}\right)\right| \leqslant|\nabla u(y)-F(Q)|+\left|F(Q)-F\left(Q_{0}\right)\right| .
$$


Because of (A.2.19) (i.e. by our choice of $l(r)$ )

$$
\mathcal{H}^{n}(B(y)) \geqslant 2\left(\delta_{\varepsilon}(r)+\delta^{\prime \prime}(r)\right) r^{n} .
$$

If we compare with the definitions (A.2.8) and (A.2.13) we see that we can choose points $Q \in B(y)$ such that

$$
\left|F(Q)-F\left(Q_{0}\right)\right|<\varepsilon \quad \text { and } \quad Q \in E(\eta) .
$$

Recall the definitions established in (A.2.9) and (A.2.10), the fact that $Q \in E(\eta)$ implies that $Q \in H\left(l_{n}, 2^{-n}\right)$ for all $n \in \mathbb{N}$. In particular choose $n$ so large that $2^{-n}<\varepsilon$, and by (A.2.9) we have that

(A.2.31) $\quad|\nabla u(X)-F(Q)| \leqslant 2^{-n}<\varepsilon \quad$ for $X \in \Gamma(Q)$ with $\delta(X) \leqslant l_{n}$.

Note that (A.2.17) ensures that for $r$ small enough $l(r)<l_{n}$ and therefore for $y \in D(r)$, $y \in \Gamma(Q)$ by (A.2.20) and

$$
|\nabla u(y)-F(Q)| \leqslant 2^{-n}<\varepsilon,
$$

which combined with (A.2.30) proves (A.2.27). From (A.2.26) and (A.2.27) we deduce that $I(r) \leqslant C \varepsilon$ for $r$ small. Because of the definition of $I(r)$ (see (A.2.25)) this means that for $r$ small

$$
\oint_{D(r)} \oint_{D(r)}\left|R\left(y_{1}, y_{2}\right)\right| d y_{1} d y_{2} \leqslant C \varepsilon r .
$$

Using (A.2.22), the fact that for $r$ small, $|u(y)| \leqslant C N_{l}\left(\nabla u\left(Q_{0}\right)\right) l(r) \leqslant \varepsilon r$ (by Lemma A.2.4 and because $\frac{l(r)}{r}$ tends to 0 as $r$ tends to 0 (see (A.2.17))) and (A.2.33) we obtain that

$$
\oint_{D(r)} \underset{D(r)}{f}\left|\left\langle F\left(Q_{0}\right), y_{2}-y_{1}\right\rangle\right| d y_{1} d y_{2} \leqslant C \varepsilon r .
$$

From this it is easy to deduce that

$$
\left|\left\langle F\left(Q_{0}\right), v\right\rangle\right| \leqslant C \varepsilon|v| \quad \text { for all } v \in T_{Q_{0}} \partial \Omega \text {. }
$$

Since (A.2.35) holds for an arbitrary $\varepsilon>0$, we conclude that $\left\langle F\left(Q_{0}\right), v\right\rangle=0$ for all $v \in T_{Q_{0}} \partial \Omega$ which proves (A.2.15).

Proof of Lemma A.2.4. - Let $y \in D(r)$, let $Q(y) \in \partial \Omega \cap B\left(Q_{0}, 2 r\right)$ be such that $\pi(Q(y))=\pi(y)$, and let $B(y)=\partial \Omega \cap B(Q(y), l(r))$. We know from (A.2.20) that

$$
\text { if } Q \in B(y) \text { then } y \in \Gamma(Q) \text { and } \delta(y) \leqslant C_{0} l(r) \text {. }
$$

Also because of (2.10) we know that $\mathcal{H}^{n}(B(y)) \geqslant 2 \delta_{\varepsilon}^{\prime}(r) r^{n}$. Using the definition (A.2.6) we see that we can find points $P \in B(y)$ so that $N_{l} \nabla u(P) \leqslant 2 N_{l} \nabla u\left(Q_{0}\right)$. Let us choose such $P \in B(y)$. Since $\Omega$ is a $\delta$-Reifenberg flat domain, by [14, Lemma 4.4] we have that

$$
u(y) \leqslant C u\left(A\left(P, C_{0} l(r)\right)\right),
$$

where $A=A\left(P, C_{0} l(r)\right) \in \Gamma(P)$, and $C_{0} l(r) / 4 \leqslant \delta(A) \leqslant C_{0} l(r)$. By (A.2.36) and using the fact that $\Omega$ is a $\delta$-Reifenberg flat domain, we know that there exists a path $\gamma \in \Gamma(P)$; with 
$\sup _{X \in \gamma} \delta(X) \leqslant C_{0} l(r)$ joining $A\left(P, C_{0} l(r)\right)$ to $A\left(P, C_{0} \delta l(r)\right)$, with length

$$
\gamma \leqslant C C_{0} l(r) \leqslant C^{\prime} l(r) .
$$

Integrating along this path we have

$$
\begin{aligned}
u\left(A\left(P, C_{0} l(r)\right)\right) & \leqslant \int_{\gamma}|\nabla u|(\zeta) d \mathcal{H}^{1}(\zeta)+u\left(A\left(P, C_{0} \delta l(r)\right)\right) \\
& \leqslant C l(r) N_{C_{0} l(r)} \nabla u(P)+u\left(A\left(P, C_{0} \delta l(r)\right)\right) \\
& \leqslant C l(r) N_{l} \nabla u(P)+u\left(A\left(P, C_{0} \delta l(r)\right)\right),
\end{aligned}
$$

for $r$ small enough because $C_{0} l(r)$ becomes much smaller than $l$. Lemma 4.1 in [14] combined with Harnack's inequality ensures that

$$
u\left(A\left(P, C_{0} \delta l(r)\right)\right) \leqslant C \delta^{\alpha} u\left(A\left(P, C_{0} l(r)\right)\right) .
$$

Therefore combining (A.2.37), (A.2.38) and (A.2.39), and given our choice of $P$ we obtain (for $r$ small)

$$
u(y) \leqslant C l(r) N_{l} \nabla u\left(Q_{0}\right) \quad \text { for } y \in D(r) .
$$

\section{A.3. Rellich's identity for chord-arc domains}

We use the machinery introduced at the beginning of this appendix to show that Rellich's identity holds for chord arc domains with small constant or for Reifenberg flat chord arc domains satisfying $\log h \in \operatorname{VMO}(\partial \Omega)$. We assume that $\Omega \subset \mathbb{R}^{n+1}$ is a bounded $\delta_{0}$ chord arc domain (or $\left(\delta_{0}, \infty\right)$-chord arc domain) with $\delta_{0}>0$ small enough to ensure that Corollary 5.2 (or Corollary 5.1 ) in [18] holds. Here $n \geqslant 2$.

LEMMA A.3.1. - Let $\Omega$ be a bounded $\delta$-chord arc domain or a $(\delta, \infty)$-chord arc domain for $\delta<\delta_{0}$ or a chord arc domain so that $\log h \in \operatorname{VMO}(\partial \Omega)$. Let $A \in \Omega$, and let $\omega^{A}$ denote the harmonic measure of $\partial \Omega$ with pole at $A$. Then if $k_{A}=\frac{d \omega^{A}}{d \sigma}$,

$$
\frac{1}{\sigma_{n}} \int_{\partial \Omega} k_{A}(Q) \frac{d \mathcal{H}^{n}(Q)}{|Q-A|^{n-1}}=-\int_{\partial \Omega} k_{A}^{2}(Q)\langle Q-A, \vec{n}(Q)\rangle d \mathcal{H}^{n}(Q)
$$

where $\sigma_{n}$ denotes the surface area of the unit sphere in $\mathbb{R}^{n+1}$, and $\vec{n}(Q)$ denotes the inward pointing unit normal.

Proof. - Let $R<\delta(A) / 8$ and $Q_{0} \in \partial \Omega$ by Lemmata A.1.2 and A.1.3 $\Omega$ can be approximated by a sequence $\left\{\Omega_{j}\right\}$ of interior chord arc domains satisfying (A.1.20), (A.1.21), (A.1.44), (A.1.45) and (A.1.46). Let $\varphi \in C_{c}^{\infty}\left(B\left(Q_{0}, R\right)\right)$, for $Q \in \partial \Omega \cap B\left(Q_{0}, R\right)$ let

$$
\alpha_{j}\left(Q_{j}\right)=Q_{j}-A-\left\langle Q_{j}-A, \overrightarrow{n_{j}}\left(Q_{j}\right)\right\rangle \overrightarrow{n_{j}}\left(Q_{j}\right)
$$

where $Q_{j}=\phi_{j}(Q)$ with $\phi_{j}$ as defined in (A.1.10) for some $\alpha<\alpha_{n}$ ( $\alpha_{n}$ as in Lemma A.1.2). Here $\overrightarrow{n_{j}}\left(Q_{j}\right)$ denotes the inward pointing unit normal to $\partial \Omega_{j}$.

As in [14] (see proof of Main Identity) we look at the expression 
(A.3.2)

$$
\begin{aligned}
\int_{\partial \Omega_{j}} & \left\langle\nabla G\left(A, Q_{j}\right), \vec{n}_{j}\left(Q_{j}\right)\right\rangle\left\langle\alpha_{j}\left(Q_{j}\right), \nabla G\left(A, Q_{j}\right)\right\rangle \varphi\left(Q_{j}\right) d \mathcal{H}^{n} \\
= & \int_{\partial \Omega_{j}}\left\langle\nabla G\left(A, Q_{j}\right), \overrightarrow{n_{j}}\left(Q_{j}\right)\right\rangle\left\langle Q_{j}-A, \nabla G\left(A, Q_{j}\right)\right\rangle \varphi\left(Q_{j}\right) d \mathcal{H}^{n} \\
& -\int_{\partial \Omega_{j}}\left\langle\nabla G\left(A, Q_{j}\right), \overrightarrow{n_{j}}\left(Q_{j}\right)\right\rangle^{2}\left\langle Q_{j}-A, \overrightarrow{n_{j}}\left(Q_{j}\right)\right\rangle \varphi\left(Q_{j}\right) d \mathcal{H}^{n} .
\end{aligned}
$$

Lemma 3.2 guarantees that for $\mathcal{H}^{n}$ a.e. $Q \in \partial \Omega$

$$
\begin{gathered}
\left\langle\nabla G\left(A, Q_{j}\right), \overrightarrow{n_{j}}\left(Q_{j}\right)\right\rangle=\left\langle\nabla G\left(A, \phi_{j}(Q)\right), \overrightarrow{n_{j}}\left(\phi_{j}(Q)\right)\right\rangle \underset{j \rightarrow \infty}{\longrightarrow} k_{A}(Q) \\
\left\langle\alpha_{j}\left(Q_{j}\right), \nabla G\left(A, Q_{j}\right)\right\rangle=\left\langle\alpha_{j}\left(\phi_{j}(Q)\right), \nabla G\left(A, \phi_{j}(Q)\right)\right\rangle \underset{j \rightarrow \infty}{\longrightarrow} 0 .
\end{gathered}
$$

Combining (A.3.3), (A.3.4) and (A.1.82) we have that for $\mathcal{H}^{n}$ a.e. $Q \in \partial \Omega$

$$
\left\langle\nabla G\left(A, \phi_{j}(Q)\right), \overrightarrow{n_{j}}\left(\phi_{j}(Q)\right)\right\rangle\left\langle\alpha_{j}\left(\phi_{j}(Q)\right), \nabla G\left(A, \phi_{j}(Q)\right)\right\rangle \varphi\left(\phi_{j}(Q)\right) J \phi_{j}(Q) \underset{j \rightarrow \infty}{\longrightarrow} 0 .
$$

Since $\sup _{X \in \Gamma_{\beta}, \delta(X) \leqslant l}|\nabla G(A, X)| \leqslant C M_{l}\left(k_{A}\right)(Q)$, where $M_{l}\left(k_{A}\right) \in L_{\text {loc }}^{2}(d \sigma)$ (by our assumption that $\delta \leqslant \delta_{0}$ or that $\log h \in \operatorname{VMO}(\partial \Omega)$ combined with Theorem 2.1,

$$
\left|\alpha_{j}\left(\phi_{j}(Q)\right)\right| \leqslant\left|A-\phi_{j}(Q)\right| \leqslant R+\delta(A)
$$

and $0 \leqslant J \phi_{j}(Q) \leqslant 1+C_{n} \delta$ by (A.1.80), the Lebesgue dominated convergence theorem ensures that

$$
\text { (A.3.6) } \begin{aligned}
\lim _{j \rightarrow \infty} & \int_{\partial \Omega_{j}}\left\langle\nabla G\left(A, Q_{j}\right), \vec{n}_{j}\left(Q_{j}\right)\right\rangle\left\langle\alpha_{j}\left(Q_{j}\right), \nabla G\left(A, Q_{j}\right)\right\rangle \varphi\left(Q_{j}\right) d \mathcal{H}^{n} \\
= & \lim _{j \rightarrow \infty} \int_{\partial \Omega}\left\langle\nabla G\left(A, \phi_{j}(Q)\right), \overrightarrow{n_{j}}\left(\phi_{j}(Q)\right)\right\rangle\left\langle\alpha_{j}\left(\phi_{j}(Q)\right), \nabla G\left(A, \phi_{j}(Q)\right)\right\rangle \varphi\left(\phi_{j}(Q)\right) \\
& \times J \phi_{j} d \mathcal{H}^{n}=0
\end{aligned}
$$

and

$$
\begin{aligned}
\lim _{j \rightarrow \infty} \int_{\partial \Omega_{j}} \varphi\left(Q_{j}\right)\left\langle\nabla G\left(A, Q_{j}\right), \overrightarrow{n_{j}}\left(Q_{j}\right)\right\rangle^{2}\left\langle Q_{j}-A, \overrightarrow{n_{j}}\left(Q_{j}\right)\right\rangle d \mathcal{H}^{n} \\
=\lim _{j \rightarrow \infty} \int_{\partial \Omega} \varphi\left(\phi_{j}(Q)\right)\left\langle\nabla G\left(A, \phi_{j}(Q)\right), \overrightarrow{n_{j}}\left(\phi_{j}(Q)\right)\right\rangle^{2} \\
\quad \times\left\langle\phi_{j}(Q)-A, \overrightarrow{n_{j}}\left(\phi_{j}(Q)\right)\right\rangle J \phi_{j}(Q) d \mathcal{H}^{n}(Q) \\
=\int_{\partial \Omega} k_{A}^{2}(Q)\langle Q-A, \vec{n}(Q)\rangle \varphi(Q) d \mathcal{H}^{n} .
\end{aligned}
$$


Now recall that for $n \geqslant 2$ (see [12, Theorem 8.29] for the bounded case, and [12, Theorems 5.6, 5.13 and 9.22] for the unbounded case)

$$
G(A, X)=\frac{1}{(n-1) \sigma_{n}}|X-A|^{1-n}-u_{A}(X)
$$

where $u_{A}$ is a harmonic function in $\Omega$, satisfying

$$
u_{\left.A\right|_{\partial \Omega}}=\left.\frac{1}{(n-1) \sigma_{n}}|X-A|^{1-n}\right|_{\partial \Omega}
$$

since by (A.1.47) $\partial \Omega_{j} \cap B\left(Q_{0}, \frac{3 R}{2}\right) \subset \Omega \cap B\left(Q_{0}, \frac{3 R}{2}\right)$

$$
\nabla G\left(A, Q_{j}\right)=-\frac{Q_{j}-A}{\sigma_{n}\left|Q_{j}-A\right|^{n+1}}-\nabla u_{A}\left(Q_{j}\right) .
$$

We now look at the term

$$
\begin{aligned}
\int_{\partial \Omega_{j}} & \left\langle\nabla G\left(A, Q_{j}\right), \overrightarrow{n_{j}}\left(Q_{j}\right)\right\rangle\left\langle Q_{j}-A, \nabla G\left(A, Q_{j}\right)\right\rangle \varphi\left(Q_{j}\right) d \mathcal{H}^{n} \\
= & -\frac{1}{\sigma_{n}} \int_{\partial \Omega_{j}}\left\langle\nabla G\left(A, Q_{j}\right), \overrightarrow{n_{j}}\left(Q_{j}\right)\right\rangle \frac{1}{\left|Q_{j}-A\right|^{n-1}} \varphi\left(Q_{j}\right) d \mathcal{H}^{n} \\
& -\int_{\partial \Omega_{j}}\left\langle\nabla G\left(A, Q_{j}\right), \vec{n}_{j}\left(Q_{j}\right)\right\rangle\left\langle Q_{j}-A, \nabla u_{A}\left(Q_{j}\right)\right\rangle \varphi\left(Q_{j}\right) d \mathcal{H}^{n} .
\end{aligned}
$$

Since for $Q_{j} \in \partial \Omega_{j} \cap B\left(Q_{0}, \frac{3 R}{2}\right)$,

$$
\left|Q_{j}-A\right| \geqslant\left|A-Q_{0}\right|-\left|Q_{j}-Q_{0}\right| \geqslant \delta(A)-\frac{\delta(A)}{4}=\frac{3 \delta(A)}{4} .
$$

Lemma 3.2, the fact that $\sup _{X \in \Gamma_{\beta}, \delta(X) \leqslant \ell}|\nabla G(A, X)| \leqslant C M_{1}\left(k_{A}\right)(Q)$, and the Lebesgue dominated convergence theorem ensure that

$$
\begin{aligned}
& \lim _{j \rightarrow \infty} \frac{1}{\sigma_{n}} \int_{\partial \Omega_{j}}\left\langle\nabla G\left(A, Q_{j}\right), \overrightarrow{n_{j}}\left(Q_{j}\right)\right\rangle \frac{\varphi\left(Q_{j}\right)}{\left|Q_{j}-A\right|^{n-1}} d \mathcal{H}^{n} \\
& \quad=\frac{1}{\sigma_{n}} \int_{\partial \Omega} \frac{k_{A}(Q)}{|Q-A|^{n-1}} \varphi(Q) d \mathcal{H}^{n} .
\end{aligned}
$$

We now look carefully at the only remaining term, namely

$$
\int_{\partial \Omega}\left\langle\nabla G\left(A, Q_{j}\right), \overrightarrow{n_{j}}\left(Q_{j}\right)\right\rangle\left\langle Q_{j}-A, \nabla u_{A}\left(Q_{j}\right)\right\rangle \varphi\left(Q_{j}\right) d \mathcal{H}^{n} .
$$

By Lemma 3.2 and using (A.3.9) we know that $\mathcal{H}^{n}$ a.e. $Q \in \partial \Omega$,

$$
\begin{aligned}
\lim _{j \rightarrow \infty} \nabla u_{A}\left(\phi_{j}(Q)\right) & =-\frac{Q-A}{\sigma_{n}|Q-A|^{n+1}}-k_{A}(Q) \vec{n}(Q) \\
& =\nabla F_{0}(A, Q)-k_{A}(Q) \vec{n}(Q),
\end{aligned}
$$


where $F_{0}(A, Q)=1 /\left((n-1) \sigma_{n}|Q-A|^{n-1}\right)$ denotes the fundamental solution of the Laplacian in $\mathbb{R}^{n+1}, n \geqslant 2$. The same argument used above ensures that

$$
\begin{gathered}
\lim _{j \rightarrow \infty} \int_{\partial \Omega_{j}}\left\langle\nabla G\left(A, Q_{j}\right), \overrightarrow{n_{j}}\left(Q_{j}\right)\right\rangle\left\langle Q_{j}-A, \nabla u_{A}\left(Q_{j}\right)\right\rangle \varphi\left(Q_{j}\right) d \mathcal{H}^{n} \\
\quad=\int_{\partial \Omega}\left\langle Q-A, \nabla F_{0}(A, Q)-k_{A}(Q) \vec{n}(Q)\right\rangle k_{A}(Q) \varphi(Q) d \mathcal{H}^{n} .
\end{gathered}
$$

Combining (A.3.2), (A.3.6), (A.3.7), (A.3.10), (A.3.11) and (A.3.13) we obtain for $\varphi \in$ $C_{c}^{\infty}\left(B\left(Q_{0}, R\right)\right)$, and $Q_{0} \in \partial \Omega$

$$
\frac{1}{\sigma_{n}} \int_{\partial \Omega} k_{A}(Q) \frac{\varphi(Q)}{|A-Q|^{n-1}} d \mathcal{H}^{n}(Q)+\int_{\partial \Omega} k_{A}^{2}(Q)\langle Q-A, \vec{n}(Q)\rangle \varphi(Q) d \mathcal{H}^{n}(Q)
$$

$(\mathrm{A} .3 .15)=-\int_{\partial \Omega}\left\langle Q-A, \nabla F_{0}(A, Q)-k_{A}(Q) \vec{n}(Q)\right\rangle k_{A}(Q) \varphi(Q) d \mathcal{H}^{n}$.

Taking a partition of unity for a neighborhood of $\partial \Omega$, and adding all the terms (corresponding to (A.3.15)) we obtain

$$
\begin{aligned}
& \frac{1}{\sigma_{n}} \int_{\partial \Omega} k_{A}(Q) \frac{d \mathcal{H}^{n}(Q)}{|Q-A|^{n-1}}+\int_{\partial \Omega} k_{A}^{2}(Q)\langle Q-A, \vec{n}(Q)\rangle d \mathcal{H}^{n} \\
& \quad=-\int_{\partial \Omega}\left\langle Q-A, \nabla F_{0}(A, Q)-k_{A}(Q) \vec{n}(Q)\right\rangle k_{A}(Q) d \mathcal{H}^{n} .
\end{aligned}
$$

We would like to remark that in the unbounded case Lemma 6.1 in [18], which is a purely technical result, ensures that all the terms are finite.

Let $H(X)=\nabla F_{0}(A, X)-\nabla G(A, X), H$ is a harmonic function in $\Omega$ (see definition Chapter 9 of [12] if $\Omega$ is unbounded), and for $\mathcal{H}^{n}$ a.e. $Q \in \partial \Omega, H(X)$ converges non-tangentially to $\nabla F_{0}(A, Q)-k_{A}(Q) \vec{n}(Q)$. Note that $V(X)=\langle X-A, H(X)\rangle$ is a harmonic function in $\Omega$, with $V(A)=0$, and such that for $\mathcal{H}^{n}$ a.e. $Q \in \partial \Omega, V(X)$ converges non-tangentially to $\left\langle Q-A, \nabla F_{0}(A, Q)-k_{A}(Q) \vec{n}(Q) \in L^{1}\left(d \omega^{X}\right)\right.$ for any $X \in \Omega$. Theorems 8.15 and 9.23 in [12] ensure that the function $\bar{V}$ defined in $\Omega$ by

$$
\bar{V}(X)=\int_{\partial \Omega}\left\langle Q-A, \nabla F_{0}(A, Q)-k_{A}(Q) \vec{n}(Q)\right\rangle d \omega^{X}(Q)
$$

is a harmonic function. Moreover for $\mathcal{H}^{n}$ a.e. $Q \in \partial \Omega, \bar{V}(X)$ converges non-tangentially to $\left\langle Q-A, \nabla F_{0}(A, Q)-k_{A}(Q) \vec{n}(Q)\right\rangle$. Therefore abusing notation slightly we have that $\Delta V=\Delta \bar{V}=0$ in $\Omega$ and $V(Q)=\bar{V}(Q)$ for $\mathcal{H}^{n}$ a.e. $Q \in \partial \Omega$.

Our next goal is to show that there exists $f \in L^{1}\left(d \omega^{A}\right)$ so that $V(X)=\int_{\partial \Omega} f(Q) d \omega^{X}(Q)$. Since $\Omega$ is a Reifenberg flat chord arc domain this will imply that $V(Q)=f(Q) \mathcal{H}^{n}$ a.e. $Q \in \partial \Omega$ (here again $V(Q)$ means the non-tangential limit of $V$ at $Q$ ). This would guarantee that $V(X)=\bar{V}(X)$ for every $X \in \Omega$, and in particular $\bar{V}(A)=V(A)=0$. To achieve this our main tool is Lemma 8.3 in [14]. To be able to use this lemma in the bounded case, and a suitable modification in the unbounded case, we need to study the behavior of the non-tangential maximal function of $V$

$$
N_{\alpha}(V)(Q)=\sup _{x \in \Gamma_{\alpha}(Q)}|V(X)|
$$


where $\alpha>0$ and

$$
\Gamma_{\alpha, \Omega}(Q)=\Gamma_{\alpha}(Q)=\{X \in \Omega:|X-Q| \leqslant(1+\alpha) \operatorname{dist}(X, \partial \Omega)\}
$$

As mentioned in Lemma 8.2 of [14], $\alpha$ does not really play a role, in the sense that $N_{\alpha}(V) \in L^{1}\left(d \omega^{A}\right)$ if and only if $N_{\beta}(V) \in L^{1}\left(d \omega^{A}\right)$ for some $\beta>0$.

Recall that $V(X)=\left\langle X-A, \nabla u_{A}(X)\right\rangle$ where $u_{A}$ is a harmonic function in $\Omega$ satisfying $G(A, X)=F(A, X)-u_{A}(X) \geqslant 0$. In particular, $0 \leqslant u_{A}(X) \leqslant F(A, X)$ for every $X \in \Omega$. Let $Y \in \Omega$ then

$$
|V(Y)| \leqslant|Y-A|\left|\nabla u_{A}(Y)\right| \leqslant C_{n}|Y-A| \frac{u_{A}(Y)}{\delta(Y)}
$$

where $\delta(Y)=\operatorname{dist}(Y, \partial \Omega)$.

From now on we assume that $Y \in \Gamma_{\alpha}(Q)$, and we consider several cases. First assume that $|Y-Q| \geqslant 2|A-Q|$ then $|Y-A| \geqslant|Y-Q|-|Q-A| \geqslant|Q-A|$ and (A.3.20) yields

$$
\begin{aligned}
|V(Y)| & \leqslant C_{n}|Y-A| \frac{F(A, Y)}{|A-Q|} \\
& \leqslant C_{n} \frac{1}{|Y-A|^{n-2}|A-Q|} \leqslant C_{n} \frac{1}{|A-Q|^{n-1}} .
\end{aligned}
$$

If $|Y-Q|<2|A-Q|$ using the fact that $V$ and $u_{A}$ are harmonic, and

$$
\mathcal{H}^{n}(\partial B(0,1))=(n+1) \omega_{n+1}
$$

we have for $2 r \leqslant \min \{\delta(Y),|Y-A|\}$

$$
\begin{aligned}
V(Y)= & \oint_{B(Y, r)} V(X) d X \\
= & \oint_{B(Y, r)} \operatorname{div}\left((X-A) u_{A}(X)\right) d X-f_{B(Y, r)}(n+1) u_{A}(X) \\
= & \frac{1}{\omega_{n+1} r^{n+1}} \int_{\partial B(Y, r)}\left\langle X-A, \frac{X-Y}{r}\right\rangle u_{A}(X)-(n+1) u_{A}(Y) \\
= & \frac{1}{\omega_{n+1} r^{n+1}} \int_{\partial B(Y, r)}\left\langle X-Y, \frac{X-Y}{r}\right\rangle u_{A}(X)-(n+1) u_{A}(Y) \\
& +\frac{1}{\omega_{n+1} r^{n+1}} \int_{\partial B(Y, r)}\left\langle Y-A, \frac{X-Y}{r}\right\rangle u_{A}(X) \\
= & \frac{1}{\omega_{n+1} r^{n}} \int_{\partial B(Y, r)} u_{A}(X) d X-(n+1) u_{A}(Y)
\end{aligned}
$$

(A.3.22)

$$
+\frac{1}{\omega_{n+1} r^{n+1}}\left\langle Y-A, \int_{\partial B(Y, r)} \frac{X-Y}{r}\left(u_{A}(X)-u_{A}(Y)\right) d X\right\rangle .
$$

Hence 
(A.3.23)

$$
\begin{aligned}
|V(Y)| \leqslant & C_{n} \frac{|Y-A|}{r} \oint_{\partial B(Y, r)}\left|u_{A}(X)-u_{A}(Y)\right| d X \\
\leqslant & C_{n} \frac{|Y-A|}{r}\left\{\oint_{\partial B(Y, r)}|G(A, X)-G(A, Y)| d X\right. \\
& +\underset{\partial B(Y, r)}{\left.\oint_{1}|F(A, X)-F(A, Y)| d X\right\} .}
\end{aligned}
$$

We look at each term separately. For $X \in \partial B(Y, r)$,

(A.3.24)

$$
\begin{aligned}
|F(A, X)-F(A, Y)| & \leqslant C_{n}|X-Y| \frac{|X-A|^{n-2}+|Y-A|^{n-2}}{|X-A|^{n-1}|Y-A|^{n-1}} \\
& \leqslant C_{n} r\left\{\frac{1}{|X-A||Y-A|^{n-1}}+\frac{1}{|Y-A||X-A|^{n-1}}\right\} \\
& \leqslant C_{n} r \frac{1}{|Y-A|^{n}} \leqslant C_{n} r \frac{1}{|Q-A|^{n-1}|Y-A|}
\end{aligned}
$$

because $|Y-A| \geqslant|A-Q|-|Q-Y| \geqslant|A-Q| / 2$, by our assumption $|Y-Q| \leqslant 2|A-Q|$. Note also that this assumption implies that $Y \in B(A, 3|A-Q|)$. Standard PDE estimates plus Harnack's inequality ensure that

$$
\oint_{\partial B(Y, r)}|G(A, X)-G(A, Y)| d X \leqslant r \sup _{Z \in \bar{B}(Y, r)}|\nabla G(A, Z)|
$$

$$
\leqslant C r \frac{G(A, Y)}{\delta(Y)} \leqslant C r M_{2 \delta(A)}\left(k_{A}\right)(Q)
$$

where $M_{2 \delta(A)}\left(k_{A}\right)(Q)=\sup _{0<s<2 \delta(A)} \delta_{B(Q, s) \cap \partial \Omega} k_{A}(Q) d \sigma$ (see proof of Lemma A.3.1).

Combining (A.3.23), (A.3.24) and (A.3.25) we obtain that for $Y \in \Gamma_{\alpha}(Q)$ if

$$
|Y-Q| \leqslant 2|A-Q|
$$

then

$$
\begin{aligned}
|V(Y)| & \leqslant C_{n}\left\{\frac{|Y-A|}{r}\right\}\left\{r M_{2 \delta(A)}\left(k_{A}\right)(Q)+\frac{r}{|Y-A||Q-A|^{n-1}}\right\} \\
& \leqslant C_{n}|Q-A| M_{2 \delta(A)}\left(k_{A}\right)(Q)+\frac{C_{n}}{|Q-A|^{n-1}} .
\end{aligned}
$$

Combining (A.3.21) and (A.3.26) we conclude that

$$
N_{\alpha}(V)(Q) \leqslant C_{n}|Q-A| M_{2 \delta(A)}\left(k_{A}\right)(Q)+\frac{C_{n}}{|Q-A|^{n-1}} .
$$

If $\Omega$ is a bounded chord arc domain with small enough constant $k_{A} \in L^{2}(d \sigma)$ and therefore $M_{2 \delta(A)}\left(k_{A}\right) \in L^{2}(d \sigma)$. This yields the following estimate

$$
\int_{\partial \Omega} N_{\alpha}(V)(Q) d \omega^{A}(Q) \leqslant C_{n}(\operatorname{diam} \Omega) \int_{\partial \Omega} M_{2 \delta(A)}\left(k_{A}\right)(Q) d \omega^{A}(Q)+C_{n} \frac{\omega^{A}(\partial \Omega)}{\delta(A)^{n-1}}
$$




$$
\begin{aligned}
& \leqslant C_{n}(\operatorname{diam} \Omega) \int_{\partial \Omega} M_{2 \delta(A)}\left(k_{A}\right)(Q) k_{A}(Q) d \sigma+\frac{C_{n}}{\delta(A)^{n-1}} \\
& \leqslant C_{n}(\operatorname{diam} \Omega) \int_{\partial \Omega}\left[M_{2 \delta(A)}\left(k_{A}\right)(Q)\right]^{2} d \sigma+\frac{C_{n}}{\delta(A)^{n-1}} \\
& \leqslant C_{n}(\operatorname{diam} \Omega) \int_{\partial \Omega} k_{A}(Q)^{2} d \sigma+\frac{C_{n}}{\delta(A)^{n-1}} .
\end{aligned}
$$

By (A.3.28), $N_{\alpha}(V) \in L^{1}\left(d \omega^{A}\right)$. Lemma 8.3 in [14] implies that $V(X)=\int_{\partial \Omega} f(Q) d \omega^{X}(Q)$ for every $X \in \Omega$ and some $f \in L^{1}\left(d \omega^{A}\right)$. As explained above this ensures that $V(X)=\bar{V}(X)$, and in particular we have (see (A.3.16), (A.3.17) and recall that $V(A)=0$ )

$$
\frac{1}{\sigma_{n}} \int_{\partial \Omega} k_{A}(Q) \frac{d \sigma(Q)}{|Q-A|^{n-1}}=-\int_{\partial \Omega} k_{A}^{2}(Q)\langle Q-A, \vec{n}(Q)\rangle d \sigma(Q) .
$$

If $\Omega$ is an unbounded domain two things remain to be done. We first show that $N_{\alpha}(V) \in L^{1}\left(d \omega^{A}\right)$, then we show there is a version of Lemma 8.3 in [14] which holds for unbounded NTA domains. From (A.3.27) we have that

$$
\begin{aligned}
\int_{\partial \Omega} N_{\alpha}(V)(Q) d \omega^{A}(Q) \leqslant & C_{n} \int_{\partial \Omega}|Q-A| M_{2 \delta(A)}\left(k_{A}\right)(Q) d \omega^{A}(Q) \\
& +C_{n} \int_{\partial \Omega} \frac{k_{A}(Q)}{|Q-A|^{n-1}} d \sigma
\end{aligned}
$$

Let $\Omega$ be an unbounded $\delta$-Reifenberg flat chord arc domain with $\delta \leqslant \delta_{0}$ and

$$
\sup _{r>0} \sup _{Q \in \partial \Omega} \theta(Q, R)<\delta_{0}
$$

for some $\delta_{0}$ small enough so that Corollary 5.1 and Lemma 6.1 in [18] hold. In this case, if $Q_{0} \in \partial \Omega$ is such that $\left|Q_{0}-A\right|=\delta(A)$ taking $M$ is large enough we obtain

$$
\begin{aligned}
\int_{\partial \Omega} \frac{k_{A}(Q)}{|Q-A|^{n-1}} d \sigma= & \int_{\partial \Omega \cap\left\{\left|Q-Q_{0}\right|<M \delta(A)\right\}} \frac{k_{A}(Q)}{|Q-A|^{n-1}} d \sigma \\
& +\int_{\partial \Omega \cap\left\{\left|Q-Q_{0}\right| \geqslant M \delta(A)\right\}} \frac{k_{A}(Q)}{|Q-A|^{n-1}} d \sigma \\
& \leqslant \frac{1}{\delta(A)^{n-1}} \omega^{A}\left(B\left(Q_{0}, M \delta(A)\right)\right)+\frac{1}{2 \delta(A)^{n-1}} \\
& \leqslant \frac{3}{2} \frac{1}{\delta(A)^{n-1}},
\end{aligned}
$$

(see [18] for details). If $\Omega$ is a Reifenberg flat chord arc domain such that $\log h \in \operatorname{VMO}(\partial \Omega)$, combining Theorem 2.1, Lemma 2.3 and a similar argument to the one presented in the proof of Lemma 6.1 in [18], we show that (A.3.31) also holds. The first term in the right hand side of (A.3.30) requires more careful attention. Let $M>4$ be a large constant to be chosen later. 


$$
\int_{\partial \Omega}|Q-A| M_{2 \delta(A)}\left(k_{A}\right)(Q) d \omega^{A}=\int_{\partial \Omega \cap\left\{\left|Q-Q_{0}\right| \leqslant M \delta(A)\right\}}|Q-A| M_{2 \delta(A)}\left(k_{A}\right)(Q) d \omega^{A}
$$

$$
+\int_{\partial \Omega \cap\left\{\left|Q-Q_{0}\right| \geqslant M \delta(A)\right\}}|Q-A| M_{2 \delta(A)}\left(k_{A}\right)(Q) d \omega^{A} .
$$

By a similar argument to the one shown in the proof of Lemma 3.1 we have

$$
\begin{gathered}
\int_{\partial \Omega \cap\left\{\left|Q-Q_{0}\right| \leqslant M \delta(A)\right\}}|Q-A| M_{2 \delta(A)}\left(k_{A}\right)(Q) k_{A}(Q) d \sigma \\
\leqslant(M+1) \delta(A) \int_{\partial \Omega \cap B\left(Q_{0}, M \delta(A)\right.} M_{2 \delta(A)}\left(k_{A}\right)^{2}(Q) d \sigma \\
\leqslant(M+1) \delta(A) \int_{\partial \Omega \cap B\left(Q_{0},(M+2) \delta(A)\right)} k_{A}^{2}(Q) d \sigma(Q) .
\end{gathered}
$$

Covering $B\left(Q_{0},(M+2) \delta(A)\right)$ by balls $\left\{B\left(Q_{i}, \frac{\delta(A)}{N}\right\}_{i=0}^{K_{n}}\right.$ with $Q_{i} \in \partial \Omega\left|Q_{i}-Q_{j}\right| \geqslant \frac{\delta(A)}{2 N}$, where $N$ is large enough, and using the fact that $\omega^{A}$ is a doubling measure, we deduce that

$$
\begin{aligned}
\int_{\partial \Omega \cap B\left(Q_{0},(M+2) \delta(A)\right)} k_{A(Q)}^{2} d \sigma(Q) & \leqslant \sum_{i=0}^{k} \int_{\partial \Omega \cap B\left(Q_{i}, \frac{\delta(A)}{N}\right)} k_{A}^{2} d \sigma \\
& \leqslant 4 \sum_{i=0}^{k} \mathcal{H}^{n}\left(\partial \Omega \cap B\left(Q_{i}, \frac{\delta(A)}{N}\right)\right)\left[f_{\partial \Omega \cap B\left(Q_{i}, \frac{\delta(A)}{N}\right)} k_{A} d \sigma\right]^{2} \\
& \leqslant C_{n} \frac{N^{n}}{\delta(A)^{n}} \sum_{i=0}^{k}\left[\omega^{A}\left(B\left(Q_{k}, \frac{\delta(A)}{N}\right)\right)\right]^{2} \\
& \leqslant C_{n} \frac{N^{n}}{\delta(A)^{n}} \sum_{i=0}^{k} \omega^{A}\left(B\left(Q_{i}, \frac{\delta(A)}{N}\right)\right) \\
& \leqslant C_{n} \frac{N^{n}}{\delta(A)^{n}} \omega^{A}\left(B\left(Q_{0},(M+3) \delta(A)\right)\right) \\
& \leqslant C_{n} \frac{N^{n}}{\delta(A)^{n}}
\end{aligned}
$$

(see Corollary 5.1 in [18] and its proof for more details).

We now look at the second term in the right hand side of (A.3.32).

$$
\begin{gathered}
\int_{\partial \Omega \cap\left\{\left|Q-Q_{0}\right| \geqslant M \delta(A)\right\}}|Q-A| M_{2 \delta(A)}\left(k_{A}\right)(Q) k_{A}(Q) \\
=\sum_{i=0_{2^{i}} M \delta(A) \leqslant\left|Q-Q_{0}\right| \leqslant 2^{i+1} M \delta(A)}^{\infty}|Q-A| M_{2 \delta(A)}\left(k_{A}\right)(Q) k_{A}(Q) d \sigma
\end{gathered}
$$

$$
\text { (A.3.35) } \leqslant 2 \sum_{i=0}^{\infty} 2^{i+1} M \delta(A) \int_{2^{i} M \delta(A) \leqslant\left|Q-Q_{0}\right| \leqslant 2^{i+1} M \delta(A)} M_{2 \delta(A)}\left(k_{A}\right)(Q) k_{A}(Q) d \sigma .
$$


As in the proof of Lemma 6.1 in [18] we look at each term

$$
\int_{2^{i} M \delta(A) \leqslant\left|Q-Q_{0}\right| \leqslant 2^{i+1} M \delta(A)} M_{2 \delta(A)}\left(k_{A}\right)(Q) k_{A}(Q) d \sigma
$$

separately.

Let $s=\delta(A)$, and $\partial \Omega \cap B(Q, r)=\Delta(r, Q)$. For $Q \in \Delta\left(2^{i+1} M s, Q_{0}\right) \backslash \Delta\left(2^{i} M s, Q_{0}\right)$, we have $|Q-A| \geqslant\left|Q-Q_{0}\right|-\left|Q_{0}-A\right|>2^{i-1} M s$. Cover $\Delta\left(2^{i+1} M s, Q_{0}\right) \backslash \Delta\left(2^{i} M s, Q_{0}\right)$ by balls $\Delta\left(\rho_{i}, Q_{j}\right), Q_{j} \in \Delta\left(2^{i+1} M s, Q_{0}\right) \backslash \Delta\left(2^{i} M s, Q_{0}\right)$ and such that the balls $\Delta\left(\frac{\rho_{i}}{5}, Q_{j}\right)$ are disjoint. Assume that $\rho_{i}>0$ is such that $N \rho_{i}=2^{i-1} M s$, where $N=2 N_{0}>2$, and $N_{0}$ is as in Corollary 5.1 or 5.2 in [18] or as in Lemma 2.3 as needed. Note that $A \in \Omega \backslash B\left(N \rho_{i}, Q_{j}\right)$, and

$$
\left(\frac{1}{\sigma\left(\Delta\left(\rho_{i}, Q_{j}\right)\right)} \int_{\Delta\left(\rho_{i}, Q_{j}\right)} k_{A}^{2} d \sigma\right)^{\frac{1}{2}} \leqslant 2 \frac{1}{\sigma\left(\Delta\left(\rho_{i}, Q_{j}\right)\right)} \int_{\Delta\left(\rho_{i}, Q_{j}\right)} k_{A} d \sigma
$$

Recall that, since $\partial \Omega$ is Ahlfors regular, there exists $C>1$ depending only on $n$ and the Ahlfors regularity constants such that $\sigma\left(\Delta\left(\rho_{i}, Q_{j}\right)\right) \geqslant C(n)^{-1} \rho_{i}^{n}$. Moreover the fact that $\Omega$ is an unbounded NTA domain, with uniform constants, guarantees that $\omega^{A}$ is uniformly doubling on $\partial \Omega \cap\left\{\left|Q-Q_{0}\right| \geqslant M s\right\}$.

Therefore the previous inequality implies that

$$
\begin{aligned}
& \int_{2^{i} M s \leqslant\left|Q-Q_{0}\right| \leqslant 2^{i+1} M s} M_{2 s}\left(k_{A}\right)(Q) k_{A}(Q) d \sigma \\
& \leqslant \sum_{j} \int_{\Delta\left(\rho_{i}, Q_{j}\right)} M_{2 s}\left(k_{A}\right)(Q) k_{A}(Q) d \sigma \leqslant \sum_{j} \int_{\Delta\left(\rho_{i}+2 s, Q_{j}\right)} k_{A}^{2}(Q) d \sigma \\
& \leqslant \sum_{j} \int_{\Delta\left(2 \rho_{i}, Q_{j}\right)} k_{A}^{2}(Q) d \sigma \leqslant 4 \sum_{j} \frac{\omega^{A}\left(\Delta\left(2 \rho_{i}, Q_{j}\right)\right)}{\sigma\left(\Delta\left(2 \rho_{i}, Q_{j}\right)\right)} \omega^{A}\left(\Delta\left(2 \rho_{i}, Q_{j}\right)\right) \\
& \leqslant C \rho_{i}^{-n} \sum_{j} \omega^{A}\left(\Delta\left(\rho_{i}, Q_{j}\right)\right) \leqslant C \rho_{i}^{-n} \sum_{j} \omega^{A}\left(\Delta\left(\frac{\rho_{i}}{5}, Q_{j}\right)\right) \\
& \leqslant C \rho_{i}^{-n} \omega^{A}\left(\Delta\left(2^{i+1} M s+\frac{\rho_{i}}{5}, Q_{0}\right) \backslash \Delta\left(2^{i} M s-\frac{\rho_{i}}{5}, Q_{0}\right)\right) .
\end{aligned}
$$

Note that $\omega^{X}\left(\Delta\left(2^{i+1} M s+\frac{2^{i-1} M s}{N}, Q_{0}\right) \backslash \Delta\left(2^{i} M s-\frac{2^{i-1} M s}{N}, Q_{0}\right)\right)$ is a non-negative harmonic function in $\Omega$, which vanishes on $B\left(2^{i} M s-\frac{2^{i-1} M s}{N}, Q_{0}\right) \cap \partial \Omega$, and whose supremum is 1 . Thus Lemmata 4.9 and 4.11 in [14] imply that

$$
\begin{aligned}
& \omega^{A}\left(\Delta\left(2^{i+1} M s+\frac{2^{i-1} M s}{N}, Q_{0}\right) \backslash \Delta\left(2^{i} M s-\frac{2^{i-1} M s}{N}, Q_{0}\right)\right) \\
& \quad \leqslant C\left(\frac{\left|A-Q_{0}\right|}{2^{i-1} M s-\frac{2^{i-2} M s}{N}}\right)^{\alpha} \leqslant C\left(\frac{1}{2^{i} M}\right)^{\alpha} .
\end{aligned}
$$


Combining (A.3.37) and (A.3.38) we obtain

$$
\int_{2^{i} M s \leqslant\left|Q-Q_{0}\right| \leqslant 2^{i+1} M s} M_{2 s}\left(k_{A}\right)(Q) k_{A}(Q) d \sigma \leqslant C \rho_{i}^{-n}\left(\frac{1}{2^{i} M}\right)^{\alpha} .
$$

Thus (A.3.35) and (A.3.39) yield

$$
\begin{aligned}
& \int_{\partial \Omega \cap\left\{\left|Q-Q_{0}\right|>M \delta s\right\}}|Q-A| M_{2 s}\left(k_{A}\right)(Q) k_{A}(Q) d \sigma \\
\leqslant & \sum_{i=0}^{\infty} C 2^{i+1} M s \rho_{i}^{-n}\left(\frac{1}{2^{i} M}\right)^{\alpha} \\
\leqslant & \sum_{i=0}^{\infty} C 2^{i+1} M s\left(\frac{N}{2^{i-1} M s}\right)^{n}\left(\frac{1}{2^{i} M}\right)^{\alpha} \\
\leqslant & s^{-(n-1)} \sum^{n-1+\alpha} \sum_{i=0}^{\infty} \frac{1}{2^{i(n-1+\alpha)}} .
\end{aligned}
$$

Combining (A.3.32), (A.3.33), (A.3.34) and (A.3.40) we have for $M=2 N=4 N_{0}$

$$
\begin{aligned}
& \int_{\partial \Omega}|Q-A| M_{2 \delta(A)}(k)_{A}(Q) d \omega^{A} \\
& \quad \leqslant C \frac{M}{\delta(A)^{n-1}}+C \frac{1}{M^{n-1+a}} \frac{1}{\delta(A)^{n-1}} \\
& \quad \leqslant C \frac{1}{\delta(A)^{n-1}} .
\end{aligned}
$$

Putting together (A.3.30), (A.3.31) and (A.3.41) we conclude that $N_{\alpha}(V) \in L^{1}\left(d \omega^{A}\right)$. Let $\Omega$ be an unbounded NTA domain and let

$$
H\left(\Omega, d \omega^{A}\right)=\left\{u \text { harmonic in } \Omega ; N_{\alpha}(U) \in L^{1}\left(d \omega^{A}\right)\right\} .
$$

LEMMA A.3.2 ([14], Lemma 8.3). - If $u \in H^{1}\left(\Omega, d \omega^{A}\right)$ then there exists $f \in L^{1}\left(d \omega^{A}\right)$ with $u(X)=\int_{\partial \Omega} f(Q) d \omega^{X}(Q)$ for all $X \in \Omega$.

Proof. - It follows the steps of the proof of Lemma 8.3 in [14]. It relies on the construction of bounded sawtooth domains inside $\Omega$, which exhaust $\Omega$. In what follows we state the results from [14] that are needed to prove Lemma A.3.2. Although the proofs there are only done for $\Omega$ bounded, since the arguments are purely geometric they can be translated to the unbounded case without any problem.

Lemma A.3.3 ([14], Lemma 6.3). - For any $\alpha>0$ there exist $\beta, \gamma>0$ such that for $Q_{0} \in \partial \Omega, s>0$ and any bounded closed set $F \subset \partial \Omega \cap B\left(Q_{0}, s\right)$ there exist an NTA domain $\Omega_{F}$ and constants $C_{1}, C_{2}>0$ so that

$$
\begin{gathered}
\partial \Omega \cap \partial \Omega_{F}=F \\
\bigcup_{Q \in F} \Gamma_{\gamma, \Omega}(Q) \cap B\left(Q_{0}, C_{1} s\right) \subset \Omega_{F} \subset \bigcup_{Q \in F} \Gamma_{\beta, \Omega}(Q) \cap B\left(Q_{0}, C_{2} s\right),
\end{gathered}
$$




$$
\text { there exists } X_{0} \in \Omega_{F} \text { so that } \operatorname{dist}\left(X_{0}, \partial \Omega_{F}\right) \simeq s \text {. }
$$

Moreover, $\omega_{\Omega}$ and $\omega_{\Omega_{F}}$ are mutually absolutely continuous on $F$.

To prove Lemma A.3.2 note that, since $N_{\alpha}(u) \in L^{1}\left(d \omega^{A}\right), u$ has a non-tangential limit $f$ for $\omega^{A}$ a.e. $Q \in \partial \Omega$, and $f \in L^{1}\left(d \omega^{A}\right)$. Choose $\beta$ associated to $\alpha$ as in Lemma A.3.3. For $\lambda>0$, let $\bar{\lambda}>0$ be so that $\omega^{A}\left(\partial \Omega \backslash B\left(Q_{0}, \bar{\lambda}\right)\right) \leqslant 1 / \lambda^{2}$. Let $F_{\lambda}=\bar{B}\left(Q_{0}, \bar{\lambda}\right) \cap\left\{Q \in \partial \Omega ; N_{\beta}(u)(Q) \leqslant \lambda\right\}$ for $Q_{0} \in \partial \Omega$ so that $\delta(A)=\left|Q_{0}-A\right|$. Construct the sawtooth region $\Omega_{\lambda}=\Omega_{F_{\lambda}}$ as in Lemma A.3.3. In particular $A \in \Omega_{\lambda}, \Omega_{\lambda} \subset \bigcup_{Q \in F_{\lambda}} \Gamma_{\beta, \Omega}(Q) \cap B\left(Q_{0}, C_{2} \bar{\lambda}\right)$, and $|u| \leqslant \lambda$ on $\Omega_{\lambda}$. As in Lemma 5.15 in [14], $\omega_{\lambda}^{A}\left(\partial \Omega_{\lambda} \backslash F_{\lambda}\right) \leqslant M \omega^{A}\left(\partial \Omega \backslash F_{\lambda}\right)$ with $M$ independent of $\lambda$ and the $\Omega_{\lambda}$ 's increase to $\Omega$. By Lemma 8.3 and Remark 5.12 in [14] there exists $f_{\lambda} \in L^{\infty}\left(d \omega_{\lambda}^{A}\right)$ so that for $X \in \Omega_{\lambda}$, $u(X)=\int_{\partial \Omega_{\lambda}} f_{\lambda} d \omega_{\lambda}^{X}$. Since $\omega_{\lambda}^{A}$ and $\omega^{A}$ are mutually absolutely continuous on $F_{\lambda}$, it follows that $f=f_{\lambda} \omega_{\lambda}$ a.e. $Q \in F_{\lambda}$. For $X \in \Omega_{\lambda} \cap B\left(Q_{0}, R\right)$ with $R>2 \delta(A)$, we have that $\omega_{\lambda}^{X}$ and $\omega_{\lambda}^{A}$ are mutually absolutely continuous. For such $X$

$$
u(X)=\int_{F_{\lambda}} f(Q) d \omega_{\lambda}^{X}(Q)+\int_{\partial \Omega_{\lambda} \backslash F_{\lambda}} f_{\lambda}(Q) d \omega_{\lambda}^{X}(Q),
$$

and by Theorem 7.1 and its corollary in [14] (see Theorem 3.1 in [18]) we have

$$
\int_{\partial \Omega_{\lambda} \backslash F_{\lambda}} f_{\lambda}(Q) d \omega_{\lambda}^{X}(Q) \leqslant \lambda \omega_{\lambda}^{X}\left(\partial \Omega_{\lambda} \backslash F_{\lambda}\right)
$$

$$
\begin{aligned}
& \leqslant C_{X, R} \lambda \omega_{\lambda}^{A}\left(\partial \Omega_{\lambda} \backslash F_{\lambda}\right) \\
& \leqslant M C_{X, R} \lambda \omega^{A}\left(\partial \Omega \backslash F_{\lambda}\right) \\
& \leqslant C_{X, R} \lambda\left\{\omega^{A}\left(\partial \Omega \backslash \bar{B}\left(Q_{0}, \bar{\lambda}\right)\right)+\omega^{A}\left(\bar{B}\left(Q_{0}, \bar{\lambda}\right) \backslash F_{\lambda}\right)\right\} \\
& \leqslant C_{X, R} \frac{1}{\lambda}+C_{X, R} \lambda \omega^{A}\left(\bar{B}\left(Q_{0}, \lambda\right) \backslash F_{\lambda}\right)
\end{aligned}
$$

$$
\leqslant C_{X, R} \frac{1}{\lambda}+C_{X, R} \int_{\bar{B}\left(Q_{0}, \bar{\lambda}\right) \cap\left\{N_{\beta}(u)(Q)>\lambda\right\}} N_{\beta}(u)(Q) d \omega^{A}(Q) .
$$

Since $N_{\alpha}(u) \in L^{1}\left(d \omega^{A}\right)$ and $N_{\alpha}(u)$ controls $N_{\beta}(u)$ we have that for $X \in B\left(Q_{0}, R\right) \cap \Omega_{\lambda}$

$$
\lim _{\lambda \rightarrow \infty} \int_{\partial \Omega_{\lambda} \backslash F_{\lambda}} f_{\lambda}(Q) d \omega_{\lambda}^{X}=0
$$

hence for $X \in B\left(Q_{0}, R\right) \cap \Omega$

$$
u(X)=\lim _{\lambda \rightarrow \infty} \int_{F_{\lambda}} f(Q) d \omega_{\lambda}^{X} .
$$

Arguing as in the proof of Theorem 5.14 in [14] we show that for every $X \in B\left(Q_{0}, R\right) \cap \Omega$

$$
u(X)=\int_{\partial \Omega} f(Q) d \omega^{X}(Q) .
$$

Since $R>2 \delta(A)$ is arbitrary, Lemma A.3.2 is established. 
We conclude the proof of Rellich's identity in the unbounded case by noting that since $N_{\alpha}(V) \in L^{1}\left(d \omega^{A}\right)$ by Lemma A.3.2 there exists $f \in L^{1}\left(d \omega^{A}\right)$ so that

$$
V(X)=\int_{\partial \Omega} f(Q) d \omega^{X}(Q) \quad \text { for all } X \in \Omega .
$$

Since $\Omega$ is a Reifenberg flat chord arc domain, $V(Q)=f(Q) \mathcal{H}^{n}$ a.e. $Q \in \partial \Omega$ which ensures that $V(X)=\bar{V}(X)$ for all $X \in \Omega$, and in particular $V(A)=\bar{V}(A)$. Thus (A.3.1) also holds in this case.

\section{REFERENCES}

[1] Alt H.W., Caffarelli L.A., Existence and regularity for a minimum problem with free boundary, J. Reine Angew. Math. 325 (1981) 105-144.

[2] David G., Wavelets and Singular Integrals on Curves and Surfaces, in: Lecture Notes in Math., Vol. 1465, Springer-Verlag, 1991.

[3] DO CARMO M., Riemannian Geometry, Birkhäuser, 1992.

[4] DAVID G., JERISON D., Lipschitz approximation to hypersurfaces, harmonic measure, and singular integrals, Indiana Univ. Math. J. 39 (1990) 831-845.

[5] David G., Semmes S., Analysis of and on Uniformly Rectifiable Sets, in: Mathematical Surveys and Monographs, AMS Series, 1993.

[6] DuRen P., The Theory of $H^{p}$ Spaces, Academic Press, New York, 1970.

[7] Evans L.C., GariePY R.F., Measure Theory and Fine Properties of Functions, in: Studies in Advanced Mathematics, CRC Press, 1992.

[8] Federer H., Geometric Measure Theory, Springer-Verlag, 1969.

[9] García-Cuerva J., Rubio de Francia J.L, Weighted Norm Inequalities and Related Topics, in: Math. Studies, Vol. 116, North Holland, Amsterdam, 1985.

[10] Garnett J.B., Jones P.W., The distance in BMO to L, Ann. of Math. 108 (1978) 373-393.

[11] Gilbarg D., Trudinger N.S., Elliptic Partial Differential Equations of Second Order, SpringerVerlag, 1983.

[12] Helms L.L., Introduction to Potential Theory, in: Pure and Applied Mathematics, Vol. 22, 1975.

[13] JERISON D., Regularity of the Poisson kernel and free boundary problems, Colloquium Math. 60-61 (1990) 547-567.

[14] JERISON D., KENIG C., Boundary behavior of harmonic functions in nontangentially accessible domains, Adv. Math. 46 (1982) 80-147.

[15] Jerison D., Kenig C., The logarithm of the Poisson kernel of a $C^{1}$ domain has vanishing mean oscillation, Trans. Amer. Math. Soc. 273 (1982) 781-794.

[16] John F., Nirenberg L., On functions of bounded mean oscillation, Comm. Pure Appl. Math. 14 (1961) 415-426.

[17] Keldysh M.V., LaVRentiev M.A., Sur la représentation conforme des domaines limités par des courbes rectifiables, Ann. Scient. Éc. Norm. Sup. 54 (1937) 1-38.

[18] Kenig C., ToRo T., Free boundary regularity for harmonic measures and Poisson kernels, Ann. of Math. 150 (1999) 369-454.

[19] Kenig C., Toro T., Harmonic measure on locally flat domains, Duke Math. J. 87 (1997) 509-551.

[20] Kenig C., Toro T., On the free boundary regularity theorem of Alt and Caffarelli, Discrete and Continuous Dynamical Systems (in press).

[21] Morrey C.B., Multiple Integrals in the Calculus of Variations, Springer-Verlag, 1966.

[22] Pommerenke Ch., On univalent functions, Bloch functions and VMOA, Math. Ann. 236 (1978) 199-208.

[23] ReIfenberg E., Solution of the Plateau problem for $m$-dimensional surfaces of varying topological type, Acta Math. 104 (1960) 1-92.

$4^{\mathrm{e}}$ SÉRIE - TOME $36-2003-\mathrm{N}^{\circ} 3$ 
[24] Semmes S., Chord-arc surfaces with small constant, II: Good parametrizations, Adv. Math. 88 (1991) 170-199.

[25] Semmes S., Analysis vs. geometry on a class of rectifiable hypersurfaces, Indiana Univ. J. 39 (1990) $1005-1035$.

[26] Simon L., Lectures on Geometric Measure Theory, Australian National University, 1983.

(Manuscrit reçu le 23 octobre 2001; accepté, après révision, le 30 avril 2002.)

\author{
Carlos E. Kenig \\ Department of Mathematics, \\ University of Chicago, \\ Chicago, IL 60637, USA \\ E-mail: cek@math.uchicago.edu

\section{Tatiana TORO} \\ Department of Mathematics, \\ University of Washington, \\ Box 354 350, \\ Seattle, WA 98195-4350, USA \\ E-mail: toro@math.washington.edu
}

